\title{
A Fully Flexible Valve Actuation System For Internal Combustion Engines
}

\author{
by \\ Junfeng Zhao \\ B.A.Sc., Tsinghua University, 2007 \\ A THESIS SUBMITTED IN PARTIAL FULFILLMENT OF \\ THE REQUIREMENTS FOR THE DEGREE OF \\ MASTER OF APPLIED SCIENCE \\ in \\ The College of Graduate Studies \\ (Mechanical Engineering)
}

THE UNIVERSITY OF BRITISH COLUMBIA

(Okanagan)

August 2009

(c) Junfeng Zhao 2009 


\section{Abstract}

Air pollution, global warming, and rising gasoline prices have lead governments, environmental organizations, and consumers to pressure the automotive industry to improve the fuel efficiency of cars.

Since alternative fuels such as hydrogen are still quite far from being commercially viable, improving the existing internal combustion engine is still an important priority. Traditional internal combustion engines use a camshaft to control valve timing. Since the camshaft is rigidly linked to the crankshaft, engineers can optimize the camshaft only for one particular speed torque combination. All other engine operating points will suffer from a suboptimal compromise of torque output, fuel efficiency, and emissions. In an engine with a camless valve actuation system, valve events are controlled independently of crankshaft rotation. As a result, fuel consumption and emissions may be reduced by $15 \% \sim 20 \%$ and torque output is enhanced in a wide range of engine speeds.

The Fully Flexible Valve Actuation (FFVA) system is our approach to construct a camless valve actuation system. Within the limits of the dynamic bandwidth of the system, it allows for fully user definable valve trajectories that can be adapted to any need of the combustion process. The system is able to achieve $8 \mathrm{~mm}$ valve lift in $3.4 \mathrm{~ms}$, which is suitable for an engine operating at $6000 \mathrm{RPM}$. The valve seating velocity is similar to conventional valve trains that achieve $0.2 \mathrm{~m} / \mathrm{s}$ at high engine speeds and $0.05 \mathrm{~m} / \mathrm{s}$ at engine idle conditions. Finally, the energy consumption measured in an experimental test bed matches the friction losses of conventional valve trains and it can further be improved by using an optimized motor.

This thesis describes the progress that has been made towards designing this technology. A design methodology is derived and important operation features of the mechanism are explained. Modeling and simulation results show significant advantages of the FFVA over previously designed electromagnetic engine valve drives. 


\section{Table of Contents}

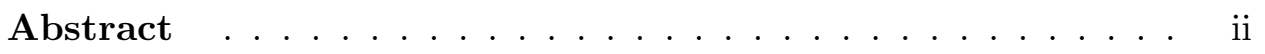

Table of Contents ................ iii

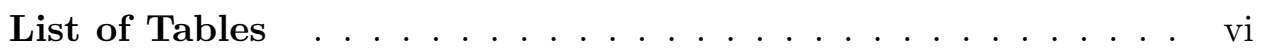

List of Figures $\ldots \ldots \ldots \ldots \ldots \ldots$ vii

Acronyms .................... ix

Nomenclature ................... $\mathrm{x}$

Acknowledgements ................ xii

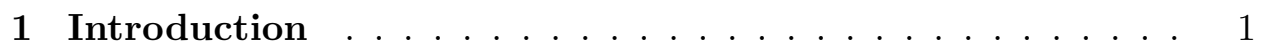

1.1 Background and Motivation ............. 2

1.2 Principles of Camless System . . . . . . . . . . . . 4

1.3 Current State of Research . . . . . . . . . . . . . 7

2 FFVA System Overview f . . . . . . . . . . . . . 12

2.1 Actuator . . . . . . . . . . . . . . . . 13

2.2 Principles of Brushless DC Motor . . . . . . . . . . 14

2.3 Valve Control Unit . . . . . . . . . . . . . . . 15 
3 Actuator Design with a Stock Motor f . . . . . . . . 18

3.1 Mechanical Optimization . . . . . . . . . . 18

3.2 Motor Selection . . . . . . . . . . . . . . . 20

3.3 Trajectory Generation . . . . . . . . . . . . . 22

3.4 Design Example . . . . . . . . . . . . . . . 26

4 Motor Optimization f. . . . . . . . . . . . 30

4.1 Stator Saturation Constraint _. . . . . . . . . . 30

4.2 Demagnetization Constraint _. . . . . . . . . . . . 32

4.3 Linearity Constraint . . . . . . . . . . . . . . . 35

4.4 Energy Constraint . . . . . . . . . . . . . . 36

4.5 Motor Design Example . . . . . . . . . . . . . . 38

5 Finite Element Analysis of DC Motor . . . . . . . . . . . 42

5.1 Brushless DC Motor Topology . . . . . . . . . . . . . . . 42

5.2 Maxwell Equations . . . . . . . . . . . . . . 43

5.3 Governing Equations in 2D Electromagnetic Field . . . . . . 44

5.4 Finite Element Simulation for BLDC Motor . . . . . . . . 46

5.5 Evaluation of Optimized Motor _. . . . . . . . . . . 49

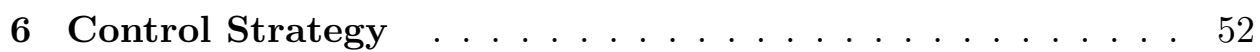

6.13 Phase Inverter . . . . . . . . . . . . . . . . 53

6.2 Position (or PD) Controller . . . . . . . . . . . . . 54

6.3 States Observer . . . . . . . . . . . . . . . 54

7 System Simulation and Experimental Validation . . . . . 61

7.1 Experimental Setup . . . . . . . . . . . . . . . 61

7.2 Simulation of Transition Performance . . . . . . . . . . . 62 
7.3 Simulation of the Robustness Towards Parameter Variations 64

7.4 Simulation of Disturbance Rejection . . . . . . . . . . 66

7.5 Simulation of Low Resolution Sensor with a Kalman Filter . 66

7.6 Experimental Results _. . . . . . . . . . . . . . 67

8 Conclusion . . . . . . . . . . . . . . . . . . 79

8.1 Summary . . . . . . . . . . . . . . . . . 79

8.2 Suggestions for Future Works . . . . . . . . . . . . . 80

Bibliography . . . . . . . . . . . . . . . 82 


\section{List of Tables}

3.1 Motor Selection . . . . . . . . . . . . . . . 27

5.1 Comparison of motor parameters . . . . . . . . . . 50

7.1 Motor Parameters . . . . . . . . . . . . . . 63

7.2 Sensitivity . . . . . . . . . . . . . . 65

7.3 Performance comparison . . . . . . . . . . . . . . 68 


\section{List of Figures}

1.1 Powertrain evolution of EU standard . . . . . . . . . . . . 2

1.2 Key technologies . . . . . . . . . . . . . . . . 3

1.3 Fuel economy solutions . . . . . . . . . . . . . . . . 4

1.4 Schematic p-V diagrams . . . . . . . . . . . . . . . 9

1.5 Honda VTEC mechanical valve actuation . . . . . . . . 10

1.6 Hydraulic valve actuation . . . . . . . . . . . . . . . 10

1.7 Solenoid valve actuation . . . . . . . . . . . . . . 11

1.8 EMVD system . . . . . . . . . . . . . . . . . 11

2.1 System structure . . . . . . . . . . . . . . . 12

2.2 Real object and 3D model of QB02302 . . . . . . . . . 13

2.3 Linkage structure between motor and valve . . . . . . . . 16

2.42 D geometry of BLDC motor . . . . . . . . . . . 17

3.1 Trajectories . . . . . . . . . . . . . . . 22

3.2 Jerk limited smooth trajectory . . . . . . . . . . . 23

3.3 Winding variation for QB02302 . . . . . . . . . . . 29

4.1 Demagnetization curve . . . . . . . . . . . . . 33

4.2 Flux density due to current and magnet . . . . . . . . . . 34

4.3 Diagram of optimization procedure . . . . . . . . . . . . . 41 


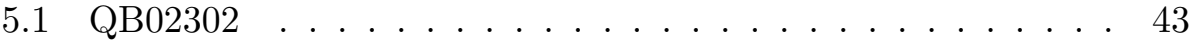

5.2 Optimized motor . . . . . . . . . . . . . . 43

5.3 Mesh result . . . . . . . . . . . . . . . . 47

5.4 Flux density distribution of the optimized motor . . . . . . 51

6.1 Diagram of control units . . . . . . . . . . . . . . . 52

6.2 Cascaded control structure . . . . . . . . . . . . . 58

6.3 Current controller . . . . . . . . . . . . . . . . . . 59

6.4 Position controller . . . . . . . . . . . . . . . 60

7.1 System hardware . . . . . . . . . . . . . . . . . 62

7.2 Simulation of displacement trajectories . . . . . . . . . . 70

7.3 Simulation of energy losses of different trajectories . . . . . 71

7.4 Transition simulation of the optimized motor . . . . . . . 72

7.5 Simulation of parameter variations . . . . . . . . . 73

7.6 Simulations of disturbance rejection . . . . . . . . . . 74

7.7 Simulation of transition response with a 7 bit sensor and various filtering strategies . . . . . . . . . . 75

7.8 Simulations of measurement error using different filtering strategies . . . . . . . . . . . . . . 76

7.9 Experimental trajectory results using QB02302 . . . . . 77

7.10 Experimental energy loss using QB02302 . . . . . . . . 78 


\section{Acronyms}

FFVA $=$ Fully Flexible Valve Actuation

$\mathrm{VVT}=$ Variable Valve Timing

EIVC=Early Intake Valve Closing

LIVC=Late Intake Valve Closing

EMCV=Electromechanical Camless Valvetrain

$\mathrm{EMVD}=$ Electromechanical Valve Drive

$\mathrm{NdFeB}=$ Neodymium Iron Boron

FEM=Finite Element Method

FEA=Finite Element Analysis

$\mathrm{LPF}=$ Low Pass Filter

$\mathrm{PWM}=$ Pulse Width Modulation 


\title{
Nomenclature
}

\author{
$r$ Rotor Radius \\ $h_{s}$ Slot Height \\ $h_{r s}$ Thickness of Stator Back \\ $r_{s i}$ Stator Inner Radius \\ $r_{s o}$ Stator Outer Radius \\ $l$ Motor Length \\ $l_{g}$ Airgap \\ $l_{g e}$ Effective Airgap \\ $l_{m}$ Magnet Thickness \\ $\beta$ Magnet Coverage Span \\ $w_{t}$ Tooth Width \\ $w_{s}$ Slot Width \\ $K_{T}$ Motor Torque Constant \\ $K_{B}$ Back EMF Constant \\ $R$ Motor Terminal Resistance \\ $L$ Motor Terminal Inductance \\ $Z$ Total Number of Conductors \\ $I$ Current in each Conductor \\ $I_{\text {total }}$ Total Current $I_{\text {total }}=Z I$ \\ $T$ Motor Torque \\ $r_{a}$ Length of Excenter Arm \\ $J_{m}$ Motor Inertia \\ $m$ Valve Mass \\ $J$ Total Inertia $J=J_{m}+m r_{a}^{2}$ \\ $\alpha$ Angular Acceleration \\ $\omega$ Angular Velocity \\ $\theta$ Rotation Angle \\ $a$ Linear Acceleration \\ $v$ Linear Velocity \\ $s$ Linear Displacement \\ $j \operatorname{Jerk}($ Derivative of $a$ )
}


E Energy Loss

$\rho$ Density of Core and Magnet

$B_{r}$ Magnet Residual Flux Density

$B_{c}$ Flux Density in Core

$B_{s}$ Saturation Flux Density

$B_{\text {go }}$ Airgap Flux Density Due to Magnet Alone

$B_{I}$ Airgap Flux Density Due to Current Alone

$B_{g}$ Airgap Flux Density $B_{g}=B_{g o}+B_{I}$

$B_{D}$ Demagnetization Flux Density

$w_{p}$ Width of Each Pole

$w_{m}$ Width of Each Magnet

$K_{s}$ Linear Current Density

$\gamma$ Angular Displacement Between the Fields Produced by the Magnet and the Stator Current

$k_{w}$ Stator Length Factor

$A_{c a}$ Copper Area per Slot

$A_{\text {slot }}$ Slot Area

$d_{w}$ Wire Diameter

$n_{w}$ Number of Wires per Slot

$k_{\text {fill }}$ Filling Factor

$p$ Number of Poles

$q$ Number of Slot per Pole per Phase

$Q$ Total Number of Slot

$\vec{H}$ Vector of Magnetizing Field

$\vec{D}$ Vector of Electric Displacement

$\vec{J}$ Vector of Free Current Density

$\vec{B}$ Vector of Magnetic Field

$\vec{A}$ Potential Vector

$\varepsilon$ Electric Permeability

$\mu$ Magnetic Permeability

$\sigma$ Electric Conductivity

$\vec{n}$ Normal Unit Vector

$P_{i}$ Proportional Gain in PI Controller

$K_{i}$ Integral Gain in PI Controller

$P_{d}$ Proportional Gain in PD Controller

$K_{d}$ Derivative Gain in PD Controller 


\section{Acknowledgements}

I would like to thank all people who have helped and inspired me during my master study.

I especially want to thank my advisor, Prof. Rudolf Seethaler, for his guidance during my research and study at University of British Columbia Okanagan. His perpetual energy and enthusiasm in research had motivated me. In addition, he was always accessible and willing to help me with my research. As a result, research life became smooth and rewarding for me. I am indebted to him more than he knows.

Prof. Holzman, Prof. Najjaran, and Prof. Koch deserve a special thanks as my thesis committee members and advisors.

Finally, I thank my family for supporting me throughout all my studies at University of British Columbia Okanagan. 


\section{Chapter 1}

\section{Introduction}

In conventional Internal Combustion (IC) engines, the timing of intake and exhaust valves is controlled by the shape and phase angle of cams. Engineers need to choose the best compromise timing among fuel economy, emissions, and torque, to design the shape of the cam. The optimization of cam shape is possible only at one engine speed. But IC engines in automobile application operate over speed and load ranges covering about an order of magnitude in each variable. This wide range of speeds and loads results in conflicting demands for the design of the lift profiles for the valves.

A fully flexible camless valve mechanism [1][2][3] allows controlling the engine load without a separate throttle and thereby avoids the associated energy loss, which will be discussed in next section. It is also known that in a conventional engine the control of valve overlap, during which both the intake and exhaust valves are open, can affect the emissions, full load and idle performance. For example, to achieve high efficiency at high speed and high load, a large amount of overlap is desired, however, this will not allow the engine to idle smoothly at low speed and low load because the residual fraction is excessive. Therefore, engine designers are starting to consider camless systems. The Fully Flexible Valve Actuation (FFVA) system in our research is a very promising technology of its kind.

Fully Flexible refers to the ability to control the duration (for how long the valve is kept open or closed), the phase (when the valve should be opened or closed), and the lift (how far does the valve move). Many presented systems have only provided duration and phase control and they are referred to as Variable Valve Timing (VVT) systems [4][5].

Flexible intake and exhaust valve mechanisms can greatly improve fuel economy, emissions, and torque of the internal combustion engine. Fuel consumption may be reduced by $15 \%-20 \%$ [6], torque output is enhanced in wide range of engine speed, and emissions may be decreased by the same ratio. 


\subsection{Background and Motivation}

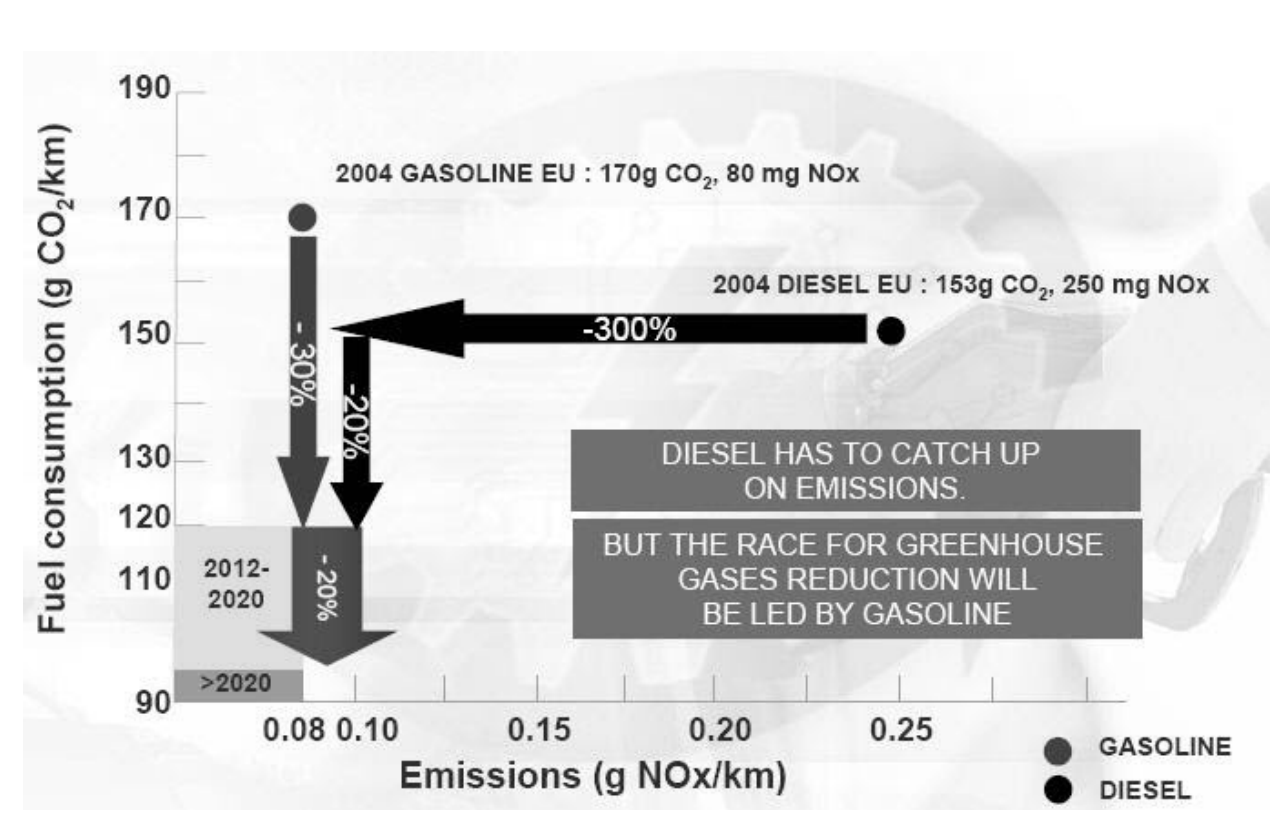

Figure 1.1: Powertrain evolution of EU standard [1]

\subsection{Background and Motivation}

The emissions of automobiles are becoming a global problem. Many countries and areas have been focusing on them and have set different standards to regulate emissions and to improve the performance of engines. Emission standards are requirements that set specific limits to the amount of pollutants that are released into the environment. While emission performance standards have been used to dictate limits for conventional pollutants such as oxides of nitrogen $N O_{x}$, this regulatory technique may also be used to regulate fuel consumption which is related to the emission of carbon dioxide $\mathrm{CO}_{2}$.

Emissions are directly influenced by the combustion process. A fuel that burns incompletely is obviously not being utilized efficiently, and unburnt fuel remains as pollutants. In both gasoline and diesel engines, the fuel is atomized very finely to ensure good combustion. But that's just one part of the story. The other is that the nature of the combustion process also influences pollutant emissions.

To address the problem of global warming, emissions of exhaust pollu- 


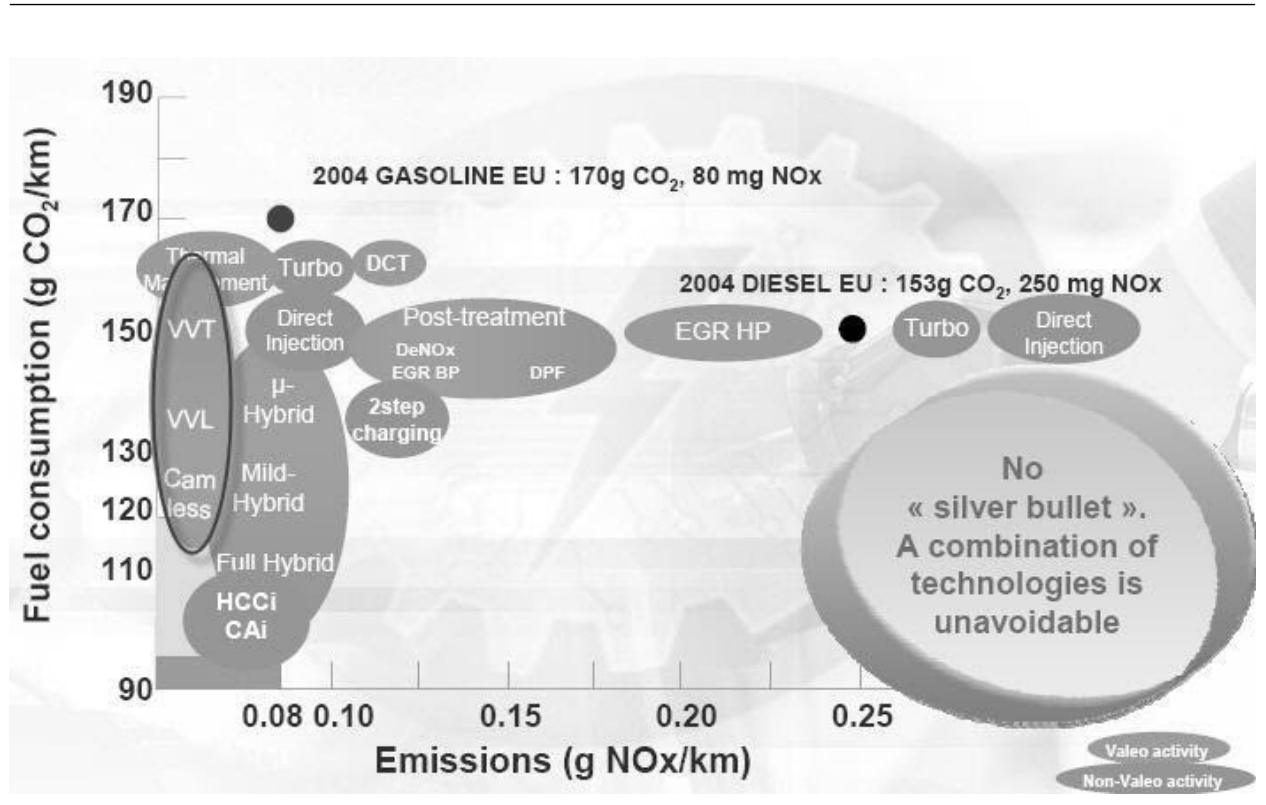

Figure 1.2: Key technologies [1]

tants and greenhouse gas from motor vehicles must be reduced [7]. Figure 1.1 shows the EU standard of powertrain evolution in the future for both gasoline engines and diesel engines, which are represented by red and black spots respectively in the picture. These objectives are challenging and conflict with each other. It can be seen that for both gasoline and diesel engines there is still a long way to go before they can meet the proposed standard. In practice, if $\mathrm{NO}_{x}$ emissions are reduced, the $\mathrm{CO}_{2}$ emissions and fuel consumption inevitably rises. With the more stringent regulations expected, new engine technologies are urgently required.

In Figure 1.2, key technologies for improving engine's performance are shown. The circled camless system, is on a very promising place on the map. Also, it can be seen from Figure 1.3 that the camless technology is the most effective, since it can improve the fuel economy by $15 \% \sim 20 \%$.

But a combination of different technologies needs to be developed, since no single one can fully satisfy the future standard. The large number of mutually interactive variables and sub-systems is a major engineering challenge, but it also provides the flexibility that is essential for converging to an optimal solution. After all, a properly matched combination of fuel, injection system, air management, and exhaust treatment should make it possible to 


\subsection{Principles of Camless System}

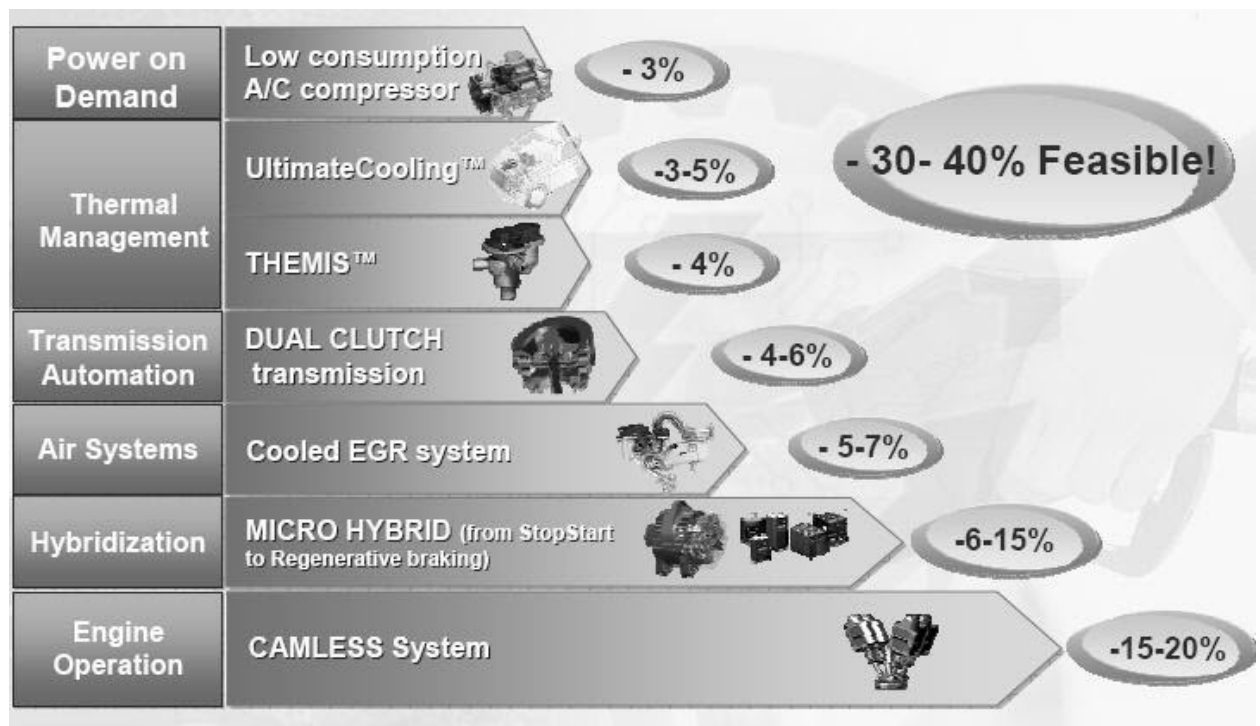

Figure 1.3: Fuel economy solutions [1]

create sufficiently stable operating conditions to meet prevailing regulatory emission and fuel economy targets.

Another advantage of camless technology is that it is compatible with all other energy saving solutions. Thus, the research on camless systems is necessary and important for the evolution of engines.

\subsection{Principles of Camless System}

In a conventional four stroke internal combustion engine, the operating cycle takes place over two revolutions of the crankshaft [9].

In the first stroke, the piston of engine starts from top dead center (TDC). At the beginning of the movement, the intake valve is already opened, which is called inlet valve opening (IVO), to let the fresh air or air-fuel mixture into the cylinder. Then after the piston has reached the bottom dead center (BDC), the intake valve is closed, which is called inlet valve closing (IVC).

The second stroke from BDC to TDC is defined as a compression stroke, 
during which both of the intake and exhaust valves are closed. Then the mixture in the cylinder is compressed when the piston moves upwards. Ignition is started when the piston is close to the end of the stroke. Then the pressure in cylinder increases rapidly.

The third stroke from TDC to BDC is defined as a power stroke. When combustion is completed, the pressure in the cylinder reaches a maximum value. Then the cylinder starts expanding, which pushes the piston to move until the exhaust valve opens, which is called exhaust valve opening (EVO). Generally before BDC, the first portion of the burned gases is allowed to be discharged.

The fourth stroke from BDC to TDC is defined as an exhaust stroke, as the piston pushes the remaining burned gases out. Near the TDC position, the intake valve opens again and the exhaust valve closes, which is called exhaust valve closing (EVC). The timing is usually slightly after the TDC position.

Figure 1.4 shows schematic p-V diagrams of a four-stroke engine with a conventional mechanical valvetrain (plot 1) and a camless systems with early intake valve closing (EIVC, plot 2) and late intake valve closing (LIVC, plot 3). The shadowed area corresponds to the work needed to induct and expel gases. These losses are referred to as pumping losses.

The pumping losses due to the throttling operation in a conventional engine with a mechanical valvetrain are quite significant [10]. A challenge in improving engine efficiency is to reduce the pumping losses while the friction loss is not excessively increased. Two strategies are available for controlling the amount of air drawn into the combustion chamber with variable valve timing systems: Early intake valve closing (EIVC) and late intake valve closing (LIVC). With EIVC, the engine load is controlled by closing the intake valve early to trap the desired amount of charge, instead of throttling the incoming charge conventionally by means of a throttle plate. Likewise, LIVC also controls engine load with reduced pumping loss, but in this case by returning unwanted charge to the intake manifold.

The comparison between pressure cycles clearly shows the advantages obtainable by adopting a camless valve actuation system in the same engine due to a significant reduction in pumping losses.

No matter whether EIVC or LIVC is implemented in the combustion process, the full controllability of each valve must be obtained by replacing the conventional valve system with a camless system, which must have some 
equivalent performance, while allowing the additional flexibility mentioned previously.

First, a camless system must allow for fast valve transitions, where the transition time refers to the time required to either open or close a valve. At an engine speed of $6000 \mathrm{rpm}$, the nominal cycle time for a conventional four stroke engine is $20 \mathrm{~ms} /$ cycle. Each cycle includes a closed-to-open transition, an open-to-closed transition, and holding phases between the transitions. The valve should be open for about one third of the cycle. Thus, the transition time at $6000 \mathrm{rpm}$ engine speed needs to be about $3.5 \mathrm{~ms}$ [11].

The second constraint is the valve seating velocity which is the valve speed when the valve hits the cylinder head after the valve closing transition. In a typical IC engine, the seating velocity is usually less than $0.3 \mathrm{~m} / \mathrm{s}[12]$ at high speed and less than $0.05 \mathrm{~m} / \mathrm{s}$ [13] at idle. Higher valve seating velocities lead to excessive noise and potentially damage to the engine. Thus, "soft landing" is an essential requirement for any valve actuation system.

The third consideration is energy loss for each transition. In a typical $2.0 \mathrm{~L}, 16$ valve, four stroke IC engine, the total energy loss of the valve train at $6000 \mathrm{rpm}$ is about $2-3 k W$ [14]. If only considering the eight intake valves (due to gas force, there is additional energy loss for each exhaust valve, but we consider intake valves only here), the power loss for each intake valve is around $1 \mathrm{~kW}$. Thus, at $6000 \mathrm{rpm}$, and $20 \mathrm{~ms} / \mathrm{cycle}$, the energy loss for each intake valve is approximately $2.5 \mathrm{~J} /$ cycle. Therefore, the average energy loss of each valve at $6000 \mathrm{rpm}$ is approximately $1.25 \mathrm{~J} /$ transition. This assumes that the power to keep the valve open or closed is minimal, which is the case for all valve actuation systems proposed to date.

The three constraints above are the main indicators of the system performance. Additional constraints (such as working temperature, applied voltage and system package size etc.) should be carefully considered before a system can be put into practical application.

Different vehicle engines run different temperatures at different conditions, but a fairly normal range is around $80^{\circ} \mathrm{C}$ to $140^{\circ} \mathrm{C}$ [15]. So the practical system should be able to work at this temperature.

Several voltage standards [16] currently exist for automotive applications: most automobiles use $12 \mathrm{~V}$ and trucks often use $24 \mathrm{~V}$. In order to reduce the size of the wire harnesses and reduce copper losses, a $42 \mathrm{~V}$ standard has been proposed but not implemented in production. In addition, Hybrid cars usually can provide approximately $200 \mathrm{~V}$ onboard voltage. For 
our investigation we will focus on $42 \mathrm{~V}$.

\subsection{Current State of Research}

In recent years, some relatively basic variable valve actuation systems have been presented in publications or even introduced into production engines. These forms mainly use mechanical, electro-hydraulic or electromagnetic valve actuation technologies.

The mechanical systems could be very simple in structure as in Figure 1.5. The simplest ones, the cam phasers, change only valve timing. Other types, slightly more complex, can change the valve lift. These mechanisms are quite simple and effective, and some of them are already on the market. But the mechanical mechanisms can only provide limited flexibility of the valve's motion, and their dynamic response is too slow for guaranteeing optimum valve timing for transient engine operations. The Honda VTEC mechanism [17] and the Toyota VVT-i system [18] are examples of mechanical variable valve timing systems.

Electro-hydraulic systems [21] [22] are conceptually quite simple. The electro-hydraulic camless systems proposed so far usually offer a continuously variable and independent control of all aspects of valve motion. Electro-hydraulic systems typically use piezo-actuated valves to control the hydraulic fluid flow that is used to displace the valve. Unfortunately, hydraulic systems suffer from viscosity changes across the required temperature range, since engine oils are typically used as the hydraulic liquid. Thus, the performance deteriorates at low temperatures. In addition, it is very difficult to achieve good energy efficiency with hydraulic systems, since there is no simple way to recover the kinetic energy of the valves when they are slowed down [23].

Electromagnetic systems are characterized by a spring system that is used to accelerate and decelerate the valve. Magnets or motors are used to hold the valves in the end positions and to compensate for friction loss as well as for combustion forces. Devices using solenoids such as in the one shown Figure 1.7, are able to generate flexible valve phase and duration, however, high valve seating velocity is difficult to control and the valve lift is limited by the structure. Nevertheless, this is a very popular technology and needs to be discussed in more detail. 
Typically, the solenoid actuator consists of a linear-moving armature with two coils and two preloaded springs. The springs can achieve rapid transition times while minimizing electrical energy input and are essential in overcoming the significant combustion pressures. The electromagnets are required for "catching" the armature at either stroke bound. In addition, they are used to overcome friction and pressure disturbances. This device needs only very little current to hold the valve at both ends. But since there is a very nonlinear relationship between force, position and current, it is very difficult to control the seating velocity when the armature approaches either end [6].

Figure 1.8 shows another electromagnetic system, in which a BLDC motor is applied to drive the valve rather than solenoids. A number of different configurations [14][25][26] exist for this design that all use springs to accelerate and decelerate the valve and a motor driven pivoting cam to provide timing. Note that the cam has a constant radius at either end of the valve motion. As a result, the motor can keep the valve at either end using zero torque. The disadvantage of this system clearly is its relatively high mechanical complexity, and the inability to adjust valve lift continuously. Nevertheless, the EMVD system demonstrates good performance on both transient time and seating velocity.

Since electromechanical systems provide better energy recovery potential, they will be used as benchmarks for the proposed FFVA system. It combines advantages of the other electromechanical systems and avoids some of their inherent problems. Like EMVD, FFVA uses a BLDC motor to generate shear force to drive the valve. This leads to a much simpler linear control system than that for the EMCV system. In contrary to EMCV and EMVD, FFVA does not use springs, but energy recovery is provided by the motor that is able to electrically feed back the breaking energy to storage capacitors. In the following sections, the system structure, design procedure and system performance are discussed in detail. 


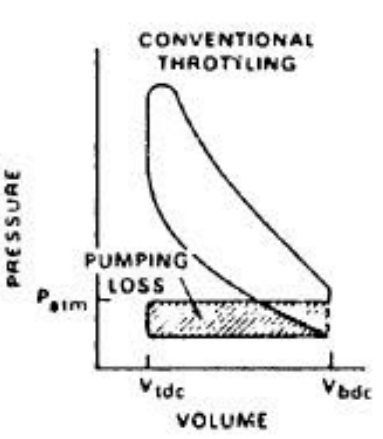

EVO = Exhaus! Valve Opens

IVO = Intake Valve Opens

EVC = Exhaust Valve Closes

IVC = Intake Valve Closes
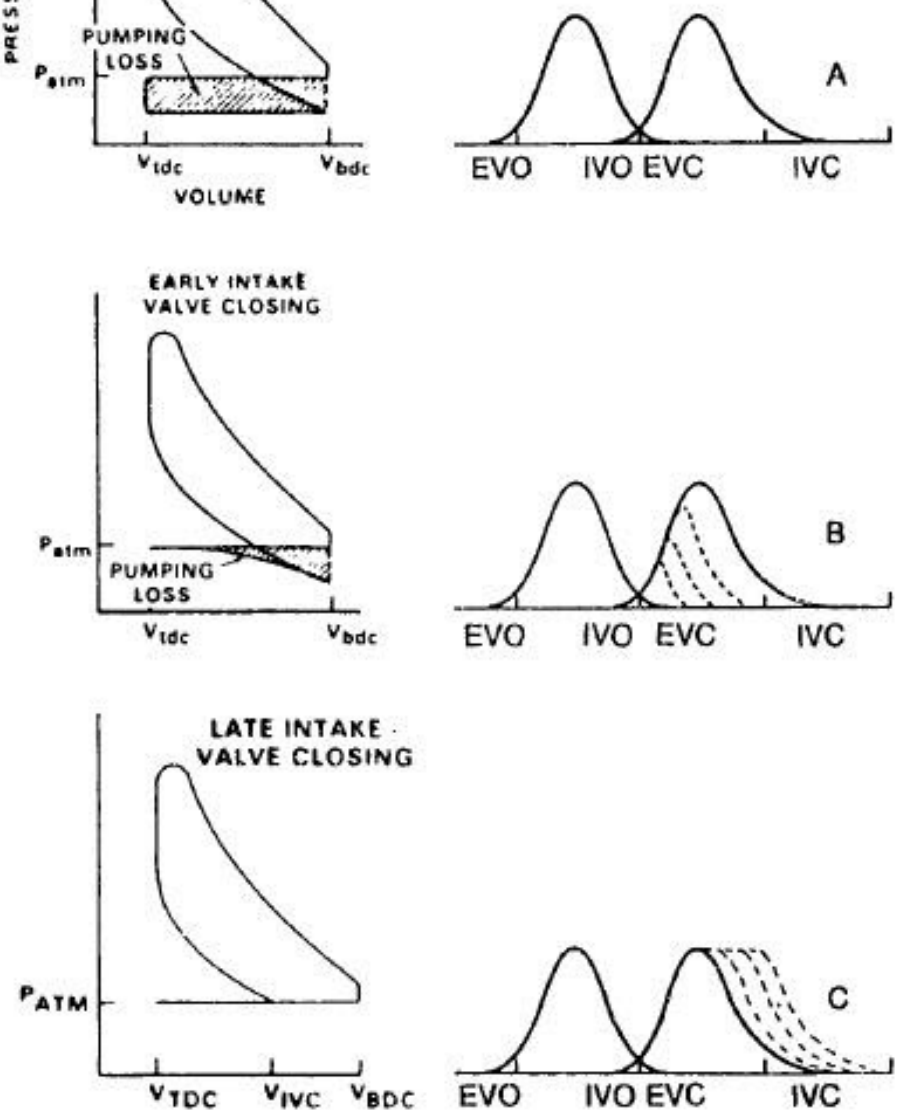

Figure 1.4: Schematic p-V diagrams [8] 


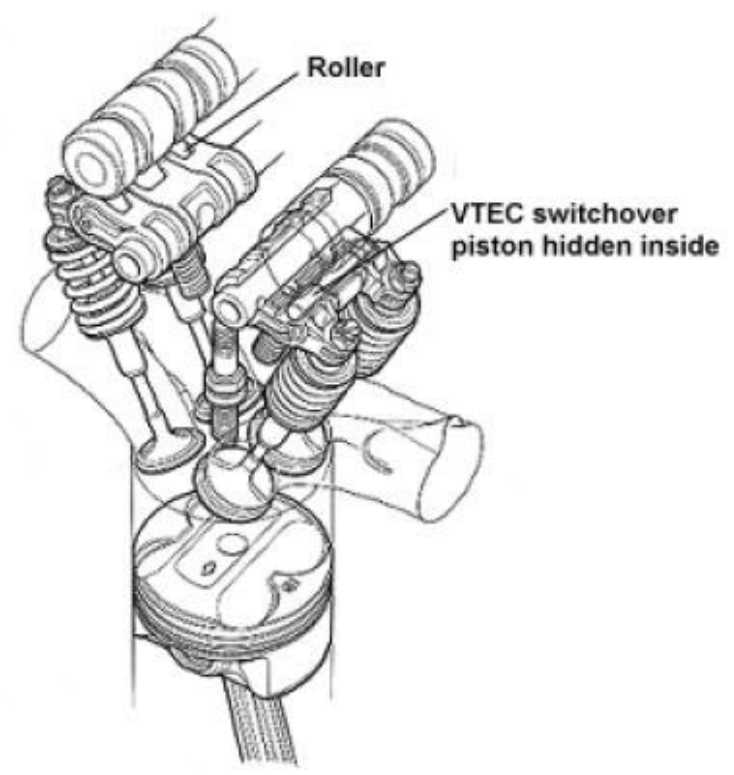

Figure 1.5: Honda VTEC mechanical valve actuation [19]

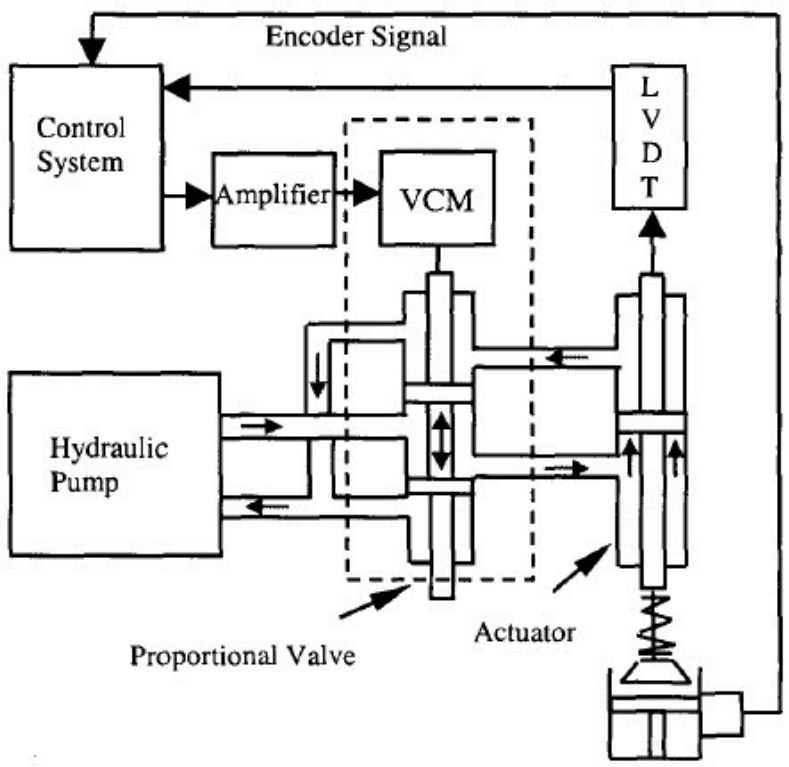

Figure 1.6: Hydraulic valve actuation [20] 


\subsection{Current State of Research}
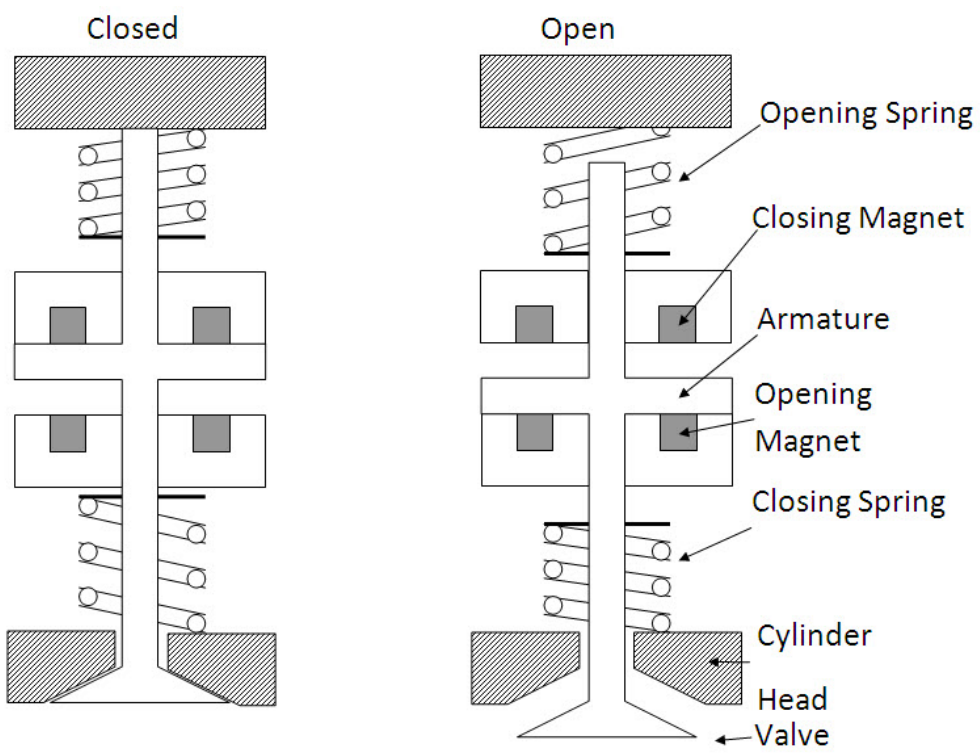

Figure 1.7: Solenoid valve actuation [24]

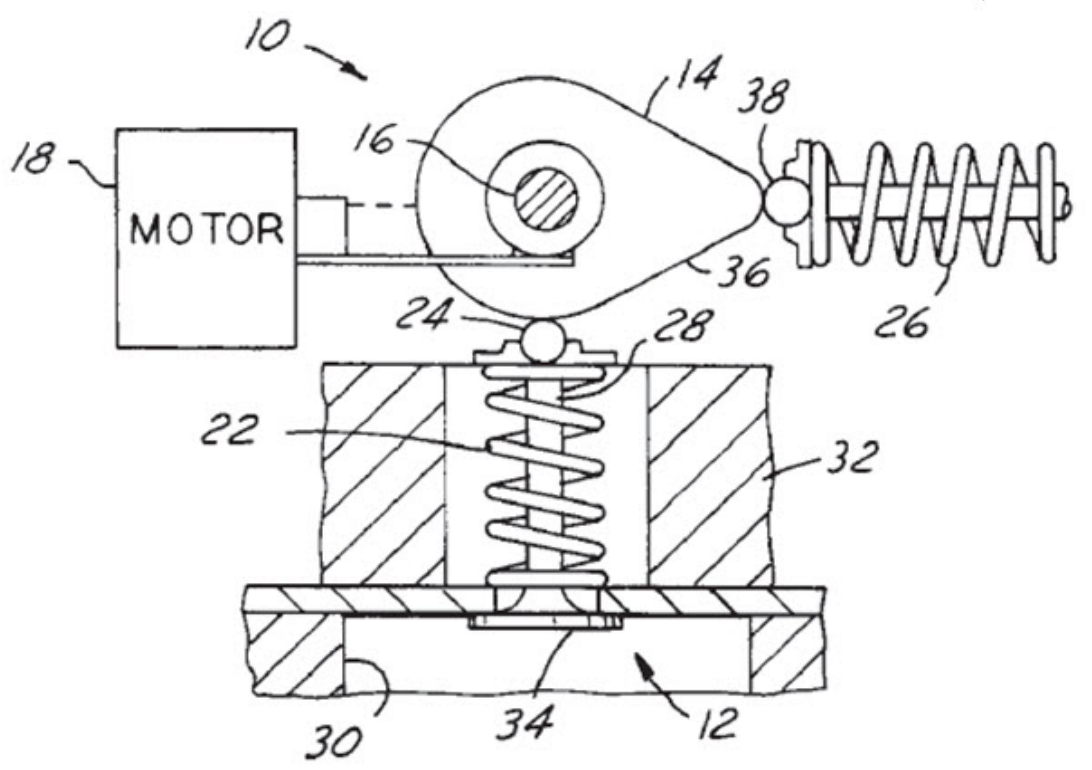

Figure 1.8: EMVD system [14] 


\section{Chapter 2}

\section{FFVA System Overview}

A schematic of the FFVA system is shown in Figure 2.1. There are three main parts: the actuation part, the valve control unit and the engine control unit.

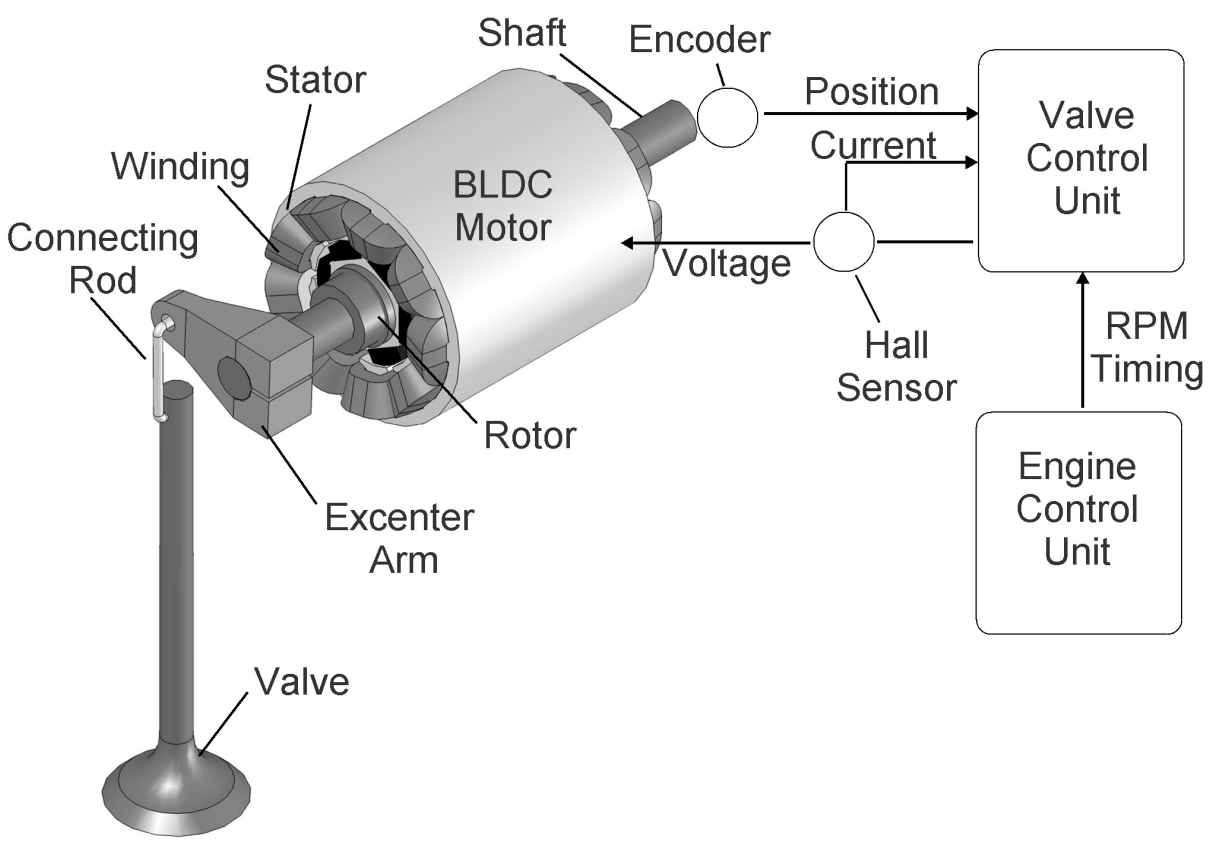

Figure 2.1: System structure

The engine control unit controls the engine operation and provides the required valve timing information to the valve control unit. The engine control unit is designed separately, and not part of this research. 

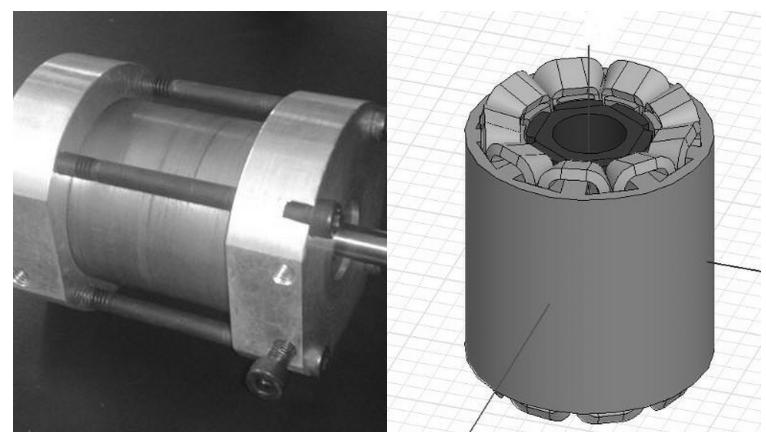

Figure 2.2: Real object and 3D model of QB02302

The valve control unit receives the desired valve timing and lift from the engine control unit and uses this information together with measured valve position and measured current in order to regulate the amount of voltage applied to the actuator.

The actuator consists of BLDC motor, a valve and a linkage structure connecting the two. A state observer, including sensors and digital filters, is connected to the actuator and used to measure the valve motion, which is then provided to the valve control unit.

\section{$2.1 \quad$ Actuator}

Figure 2.3 shows a detailed picture of the linkage between motor and valve. An excenter arm of length, $r_{a}$, is attached to the motor's shaft, which in turn is connected to the valve through a small bracket. This structure transfers the motor's angular movement to the valve's linear movement:

$$
a=\alpha r_{a}
$$

If the rotor angle is small, the relationship between lift and rotor angle can be assumed linear. The excenter arm is made of aluminium and its inertia can be neglected when compared to the rotor inertia and the valve mass. The motor torque $T$ is used to accelerate the rotor with an inertia, $J_{m}$, and the valve with a reflected inertia $m r_{a}^{2}$, where $m$ is the mass of the valve:

$$
T=\left(J_{m}+m r_{a}^{2}\right) \alpha
$$


The figure also shows several parameters, which play an important role in determining the system's performance. These parameters, especially the rotor angle $\theta$ and excenter arm length $r_{a}$, will be discussed in the chapter of optimization.

\subsection{Principles of Brushless DC Motor}

As shown above, a brushless DC motor is the key part of the FFVA system. The basic structure of a permanent magnet brushless DC motors has three elements: a stator with windings, a rotor with permanent magnets attached to it, and a sensor to measure the rotor position. A picture of QB02302 [27] and its 3D model is given in Figure 2.2.

The motor's torque constant and other properties are all related to the structure and material of the motor. Figure 2.4 provides the dimensioning details of an interior rotor BLDC motor (2D geometry of motor QB02302). The rotor radius $r$, the slot height $h_{s}$ and the length of the motor $l$ are treated as variables for an optimized design goal in subsequent chapters. The airgap $l_{g}$ and magnet thickness $l_{m}$ are selected to give sufficient flux density in the airgap. Magnets are glued on the rotor surface. The coverage of the magnets on the rotor is called span, represented by $\beta$. In our application, the span is about 0.8 . Inside the stator, the tooth width $w_{t}$ and the slot width $w_{s}$ are almost the same.

To understand how these elements work as a motor, consider some elementary magnetics [28]. When a current carrying wire is placed in a magnetic field so that the current flow is perpendicular to the direction of the field, a force is exerted between the field producing element and the wire.

The electromagnetic model of the actuator is approximated by an equivalent linear single phase DC-motor model. The mathematical model of the BLDC motor is based on the following assumptions: 1) stator resistances of all the three phases are equal and the self and mutual inductances are constant; 2) the motor is operated within the rated condition and hence the saturation effect due to current level is neglected; 3) iron losses are negligible.

This approach is followed, since most motor specifications are based on this model and we want to be able to select an optimum motor from the manufacturer's motor specifications. The motor current $I$, is defined by a first order differential equation in terms of the applied voltage $U$, and 
the back EMF voltage $K_{B} \omega$, the winding resistance $R$, and the winding inductance $L$ :

$$
\frac{d I}{d t}=\frac{1}{L}\left(U-K_{B} \omega-I R\right)
$$

The acceleration capability of the device is the torque $T$, which is proportional to the current $I$ :

$$
T=K_{T} I
$$

The maximum or peak torque available for a particular motor is given by:

$$
T_{\max }=K_{T} I_{\max }
$$

This is not the continuous torque available, which is usually constrained by heat generation of the windings, but the value of torque that is constrained by saturation of the core or demagnetization of the permanent magnets on the rotor. It is important to recognize that actuating valves is an intermittent operation in nature and requires large accelerations over short periods of time. Thus, the performance is governed by the peak torque and resistive copper losses of the motor rather than the continuous torque rating of the motor. For small automotive engines, the actuator motor should consume less than $120 \mathrm{~W}$ at $6000 \mathrm{rpm}$ and provide enough transient torque in order to achieve valve openings or closings in $3.5 \mathrm{~ms}$. Chapter 3 will show how to select a suitable stock motor for this application. Chapter 4 provides a strategy to modify the stock motor in order to achieve optimum performance.

\subsection{Valve Control Unit}

The main function of the valve control unit is to move the valve from the closed to the opened position (and vice-versa) avoiding noise, which is caused by nonzero seating velocity. This is achieved using a cascaded tracking controller with feed forward.

In Chapter 3, valve trajectories for minimizing energy and maximizing acceleration will be derived, and in Chapter 6 the design of the tracking controller will be described. 


\subsection{Valve Control Unit}

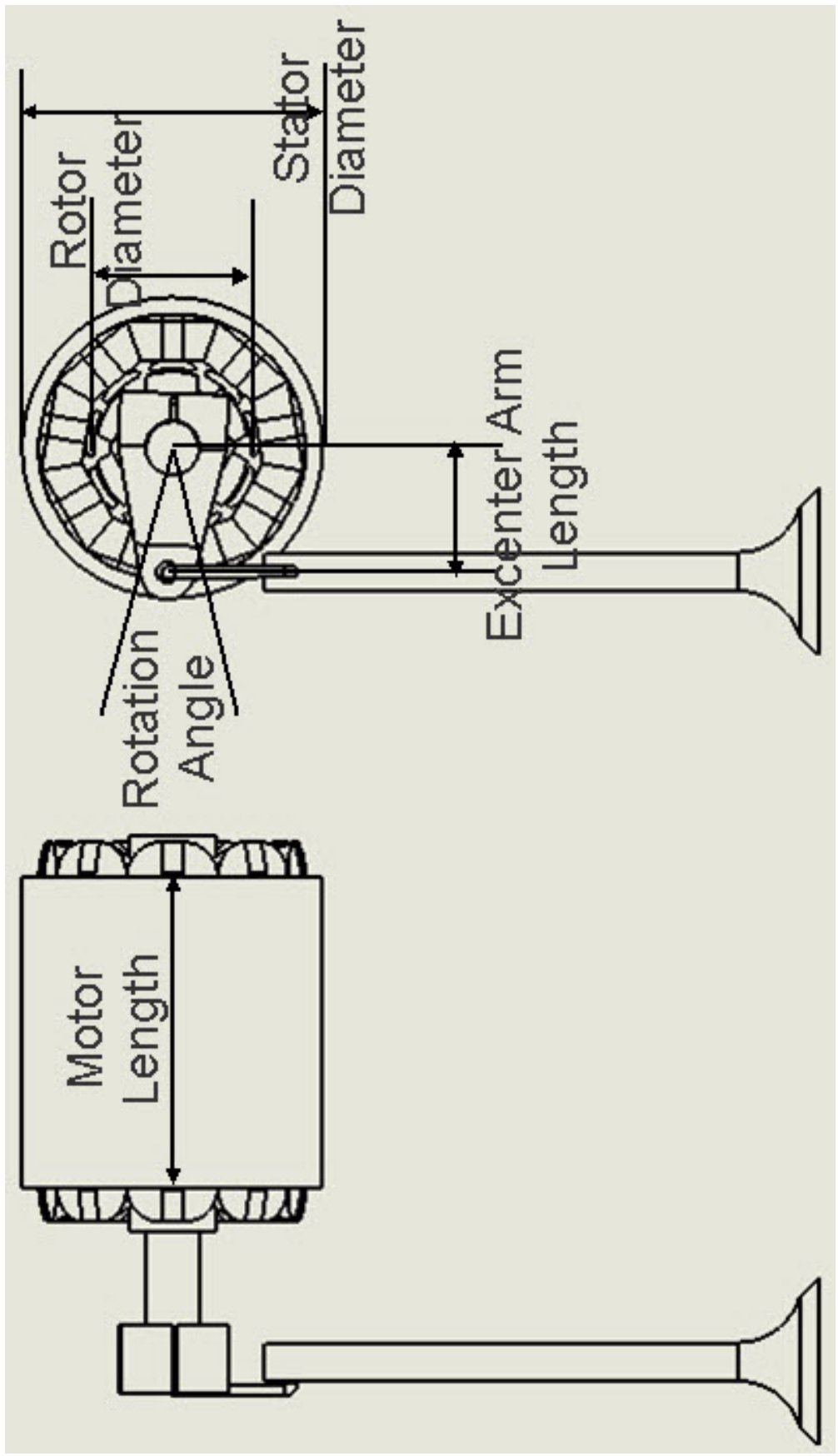

Figure 2.3: Linkage structure between motor and valve 


\subsection{Valve Control Unit}

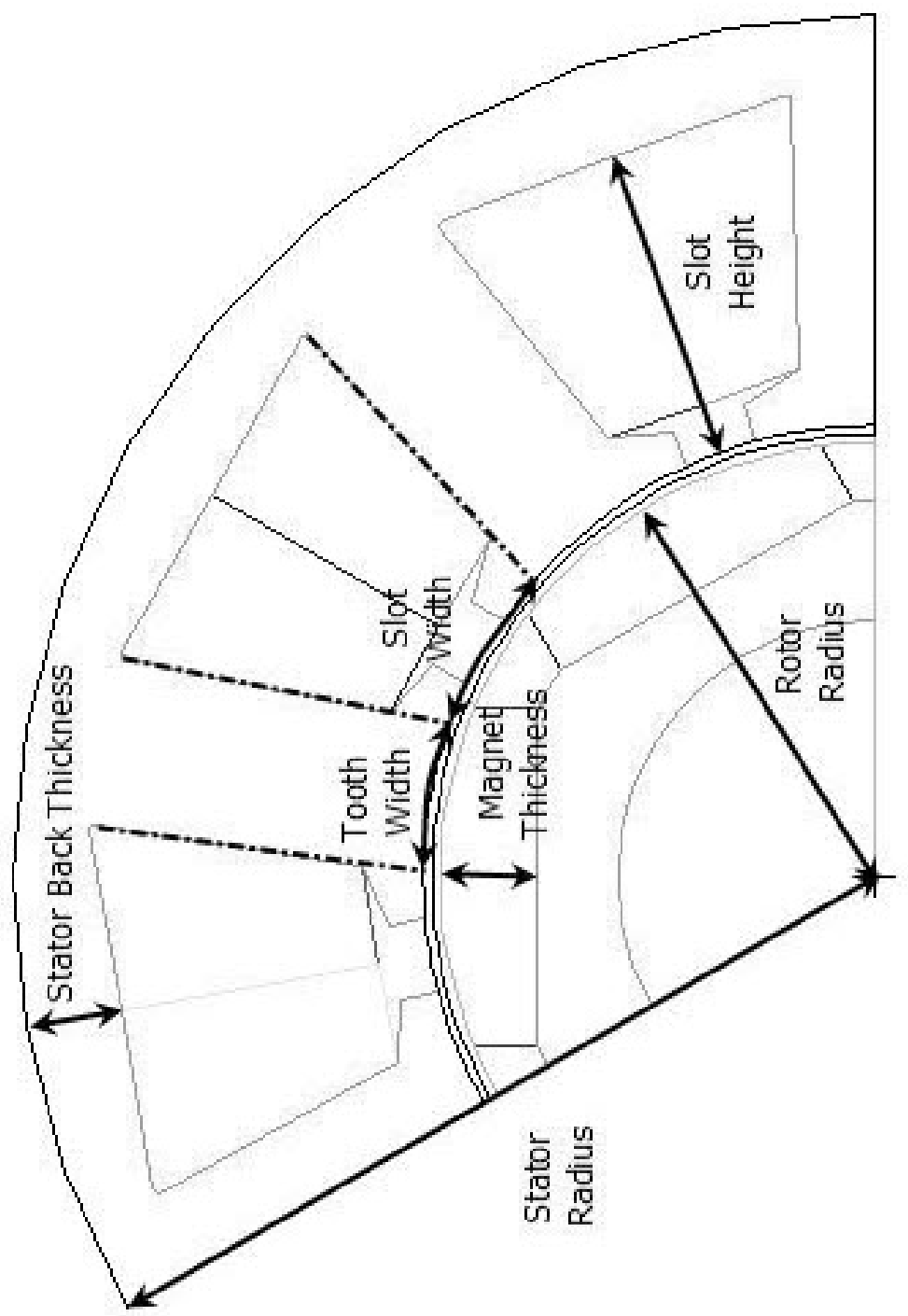

Figure 2.4: 2D geometry of BLDC motor 


\section{Chapter 3}

\section{Actuator Design with a Stock Motor}

The FFVA system is a servo system that uses a lightweight mechanical linkage structure to transfer the motor rotation to the valve translation. Typically, high speed servo systems operate at the accelerations less than $40 \mathrm{~g}$. The application shown here requires acceleration in the order of $250 \mathrm{~g}$. At the same time, energy consumption needs to be minimized in order to ensure that fuel consumption reductions gained through the introduction of variable valve control are not offset by the power consumption of the valve actuation system. To achieve these goals, the mechanical linkage, the electric motor, and the valve trajectories are optimized in the following sections.

\subsection{Mechanical Optimization}

In the FFVA system, the motor's rotation is converted to valve's vertical motion through an arm-like structure called the excenter arm. The length of the excenter arm, $r_{a}$, plays an important role in the performance of the actuation system. This section outlines how the arm length can be optimized to provide maximum acceleration and minimum energy consumption.

Maximum power transfer will occur in a mechanical system if the inertia of the load matches the inertia of the motor. That is, for a specific motor, if load inertia reflected to the motor shaft can be made to match the motor inertia, disregarding added inertia and inefficiency of the reducer, power transfer will be optimized and maximum acceleration of the load will result. The proof for this is detailed [29].

The motor torque, $T$, is used to accelerate the rotor with inertia, $J_{m}$, and the valve with a reflected inertia of $m r_{a}^{2}$, where $m$ is the mass of the 
valve:

$$
T=\left(J_{m}+m r_{a}^{2}\right) \alpha
$$

This equation can be rearranged to provide the rotor acceleration, $\alpha$ :

$$
\alpha=\frac{T}{J_{m}+m r_{a}^{2}}
$$

The valve acceleration is $a=\alpha \cdot r_{a}$. Taking the derivative of $a$ with respect to $r_{a}$ results in:

$$
\frac{d a}{d r_{a}}=\frac{d}{d t}\left(\frac{T r_{a}}{J_{m}+m r_{a}^{2}}\right)=\frac{T\left(J_{m}+m r_{a}^{2}\right)-2 T m r_{a}^{2}}{\left(J_{m}+m r_{a}^{2}\right)^{2}}
$$

By setting the derivative equal to zero, one finds the excenter arm length that provides maximum acceleration:

$$
r_{a}=\sqrt{\frac{J_{m}}{m}}
$$

It will now be shown that the same excenter arm length also provides minimum energy consumption, given a desired valve acceleration profile. When the motor's rotation angle, denoted as $\theta$, is small enough, the valve motion can be regarded as linear. Then the relationship between valve's linear displacement $s$ and motor's rotational displacement $\theta$ can be expressed as:

$$
s=r_{a} \theta
$$

Thus,

$$
\frac{d^{2} s}{d t^{2}}=r_{a} \cdot \alpha
$$

where $\alpha$ is angular acceleration. The torque that the motor provides is:

$$
T=J \alpha=\frac{J}{r_{a}} \frac{d^{2} s}{d t^{2}}
$$

where $J$ is the total inertia including motor inertia $J_{m}$ and load inertia $m r_{a}^{2}$ :

$$
J=J_{m}+m r_{a}^{2}
$$

If the motor constant is $K_{T}$, the current required is:

$$
I=\frac{T}{K_{T}}=\frac{J \alpha}{K_{T}}=\frac{J}{K_{T} r_{a}} \frac{d^{2} s}{d t^{2}}
$$


Now consider the motor's copper loss, which depends on current in the form of:

$$
E=\int I^{2} R d t=\left(\frac{J}{K_{T} r_{a}}\right)^{2} R \int\left(\frac{d^{2} s}{d t^{2}}\right)^{2} d t=\left(\frac{J_{m}+m r_{a}^{2}}{K_{T} r_{a}}\right)^{2} R \int\left(\frac{d^{2} s}{d t^{2}}\right)^{2} d t
$$

Take the derivatives on both sides:

$$
\frac{\partial E}{\partial r_{a}}=\frac{2 R}{K_{T}^{2}}\left(m-\frac{J_{m}}{r_{a}^{2}}\right)\left(\frac{J_{m}+m r_{a}^{2}}{K_{T} r_{a}}\right)^{2} R \int\left(\frac{d^{2} s}{d t^{2}}\right)^{2} d t
$$

Then the optimal arm length is found as:

$$
r_{a}=\sqrt{\frac{J_{m}}{m}}
$$

which is exactly the same as the optimal arm length for maximum acceleration derived previously.

This suggests that matching the inertia of the load with the inertia of the motor will simultaneously provide maximum acceleration and minimum energy consumption.

\subsection{Motor Selection}

Few off-the-shelf motors are designed for the highly dynamic operating conditions found in the FFVA system. However, it is very important for a successful implementation that the motor operates with high efficiency. Thus, using the findings from the mechanical optimizations, criteria for selecting a motor that provides maximum valve acceleration and minimum energy consumption are derived in the following section.

The mechanical optimization assumed that the rotor motion would be small enough in order to ensure a linear relationship between valve and motor motion. Using this assumption, the identical Equations 3.4 and 3.12 describe the optimal excenter arm length. One can now go backwards and describe the minimum motor inertia required in order to achieve a rotor angle smaller than $\theta_{\max }$, for a maximum valve motion, $s_{\max }$, and a valve mass, $m$ :

$$
J_{m} \geqslant m\left(\frac{s_{\max }}{\theta_{\max }}\right)^{2}
$$




\subsection{Motor Selection}

Since minimizing the size of the motor reduces cost and facilitates packaging the motor in the cylinder head, one would usually choose motors with inertia close to the linearity constraint.

In addition to the linearity requirement, the motor is also required to provide large acceleration. Substituting Equation 3.4 back into Equation 3.2 provides an equation for the maximum valve acceleration in terms of motor and valve parameters:

$$
a_{\max }=\frac{T_{\max } r_{a}}{J_{m}+m r_{a}^{2}}=\frac{T_{\max }}{2 \sqrt{J_{m} m}}
$$

This expression indicates that maximum acceleration is achieved by minimizing the valve mass. It also shows that the ratio of maximum torque over square root of motor inertia should be maximized. The relationship can be used to compare and select stock motors from their specification data sheets.

In addition to large accelerations, the motor also needs to minimize copper losses.

If the optimal arm length is substituted back into Equation 3.10 for energy consumption, the following equation is obtained:

$$
E=4 m \times \frac{J_{m} R}{K_{T}^{2}} \times \int\left(\frac{d^{2} s}{d t^{2}}\right)^{2} d t
$$

This equation indicates that there are three parts of the actuator that need to be optimized in order to minimize copper losses.

- Energy consumption is directly related to valve mass $m$. A low mass valve will significantly reduce energy consumption.

- The energy cost term of the motor consists of three parameters, the motor inertia multiplied by the electrical resistance divided by the square of the motor torque constant. The energy cost term provides a convenient guideline to compare and select stock motors using their specification data sheets. Chapter 4 will derive an optimum design procedure for the motor.

- The last term in Equation 3.15 is the integral of the acceleration trajectory of the valve. The next section will show how to design energy optimal trajectories. 


\subsection{Trajectory Generation}

The usual requirement for a valve trajectory is that the valve needs to travel the desired lift within a prescribed transition time $t_{4}$, which is given by the engine control unit. Both lift, and transition time will vary with changing engine speeds and engine torque requirements. Thus, a simple online algorithm that generates energy optimal valve trajectories is required. The problem can be solved by calculus of variations [30], which leads to the following valve acceleration [31]:

$$
a(t)=a_{\max }\left(1-\frac{2 t}{t_{4}}\right)
$$

where $a_{\max }$ is the maximum acceleration required to achieve the desired

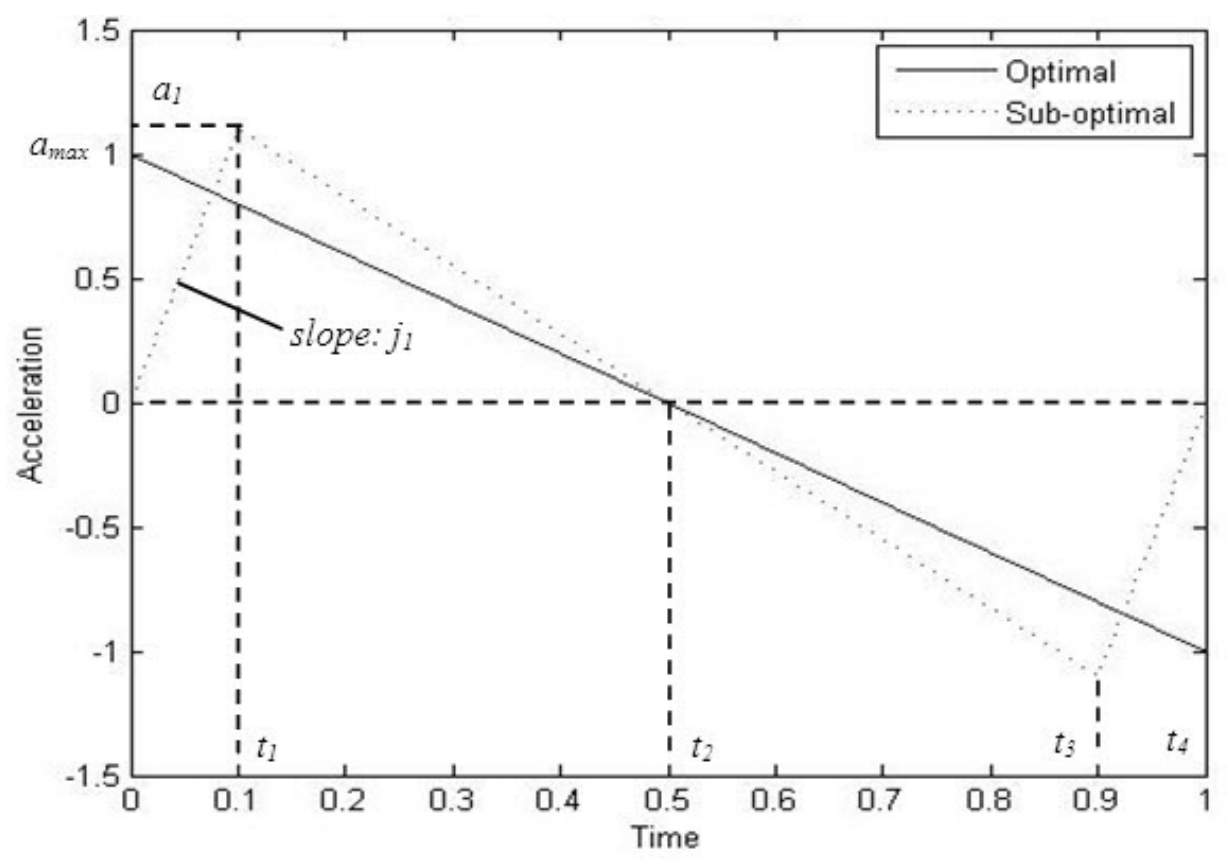

Figure 3.1: Trajectories

valve lift and transition time with minimum energy consumption. The wave form is shown as the plot of "Optimal" in Figure 3.1 and the resistive copper 


\subsection{Trajectory Generation}

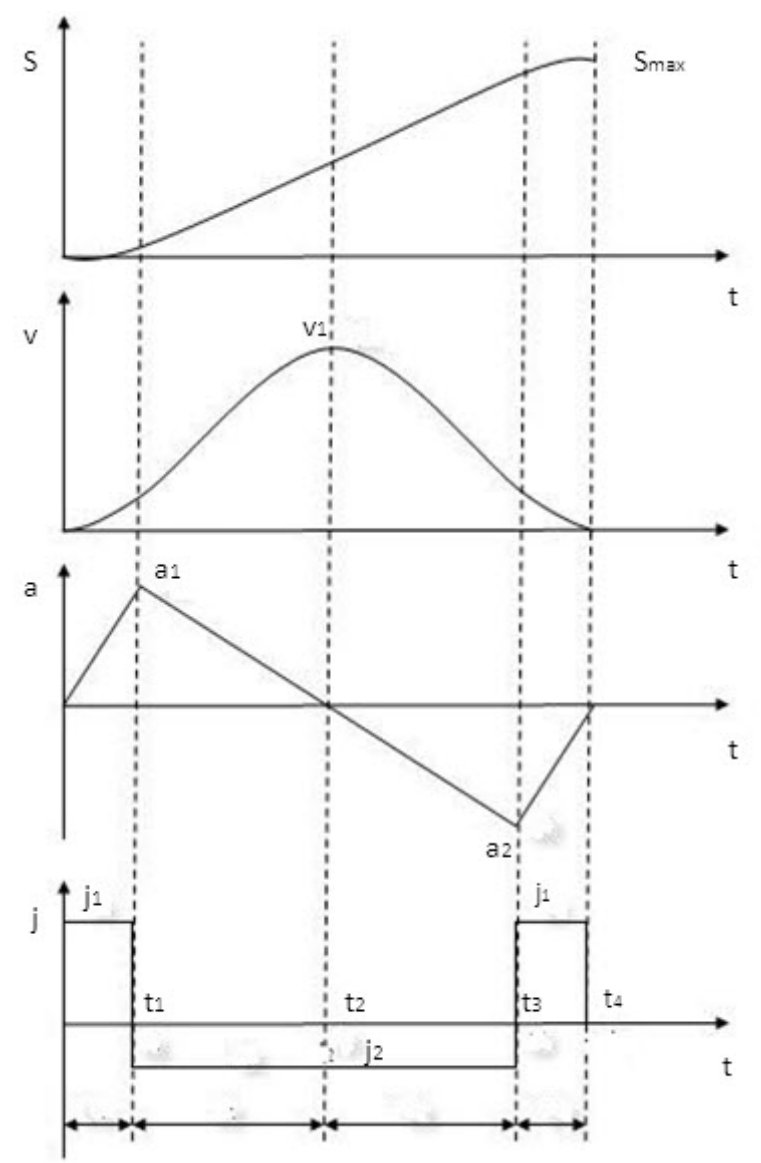

Figure 3.2: Jerk limited smooth trajectory

losses for this trajectory can be expressed as:

$$
E_{\text {opt }}=\int I^{2} R d t=\frac{3 s_{\max }^{2}\left(J_{m}+m r_{a}^{2}\right)^{2} R}{2 t_{2}^{3} r_{a}^{2} K_{T}^{2}}
$$

where $s_{\max }$ is the maximum valve travel, and $t_{2}=t_{4} / 2$ is half the valve travel time.

In practice, the optimal trajectory suggested above is not feasible due to two physical constraints.

First, maximum current constrains the available acceleration for a motor 
with constant $K_{T}[32]$ :

$$
a=\frac{T r_{a}}{J}=\frac{I K_{T} r_{a}}{J}
$$

Secondly, the ideal trajectory contains acceleration discontinuities, or infinite jerk at the beginning and at the end of the valve movement. However, jerk is limited by the driving voltage:

$$
j=\frac{K_{T} r_{a}}{J L}\left(U_{\max }-K_{T} \frac{v}{r_{a}}-R \frac{a J}{K_{T} r_{a}}\right)
$$

If the motor parameters are fixed, then the voltage becomes the limiting factor for jerk. The optimal trajectory can be followed only when the motor can provide infinite jerk, which requires infinite voltage.

The remaining section then outlines a procedure for imposing a jerk limited smooth trajectory. The kinematic profiles used in trajectory generation are illustrated in Figure 3.2. The suboptimal acceleration has a triangular profile with maximum slopes (i.e. jerk values) at the start and at the end of the valve motion. It requires a slightly higher $a_{\max }$ than the optimal trajectory, which can be seen from Figure 3.1.

Given a time $t_{1}$ during which the initial maximum jerk $j_{1}$ is applied, the value of this maximum jerk is defined as:

$$
j_{1}=\frac{3 s_{\max }}{2 t_{2}-t_{1}} t_{1} t_{2}
$$

The energy lost in the copper windings during a single transition from closed to open or from open to closed, with the sub-optimal trajectory is given by:

$$
E_{\text {subopt }}=\int I^{2} R d t=\frac{6 s_{\text {max }}^{2} J^{2} R}{2 t_{2}\left(t_{1}-t_{2}\right)^{2} r_{a}^{2} K_{T}^{2}}
$$

It is interesting to note the ratio of energies of the optimal to the suboptimal trajectory. As expected the ratio increases for larger values of $t_{1}$, which leads to the conclusion that a motor with rather small inductance is required in order to achieve good energy efficiency:

$$
\frac{E_{\text {opt }}}{E_{\text {subopt }}}=\frac{4 t_{2}^{2}}{\left(t_{1}-2 t_{2}\right)^{2}}
$$

We now aim our attention towards finding the minimum value for $t_{1}$. Equation 3.19 indicates that during the initial period where $j=j_{1}$, the 
voltage must be continuously increasing, since the jerk is constant and both velocity and acceleration are increasing. Thereafter, the voltage decreases, since jerk is reversed. Thus, it is expected that the maximum voltage will take place at $t=t_{1}$. Equations 3.19 and 3.20 for $t=t_{1}$ lead to a quadratic equation in terms of $t_{1}$ :

$$
\left(3 K_{T}^{2} s_{\max }+2 K_{T} t_{2} r_{a} U\right) t_{1}^{2}+6 s_{\max } L J+\left(6 s_{\max } R J-4 K_{T} t_{2}^{2} r_{a} U\right) t_{1}=0
$$

The solution to this equation represents the minimum and maximum value for $t_{1}$. The smaller of the two values is the minimum energy solution for the triangular trajectory. Note that the fastest trajectory possible with a given motor and fixed supply voltage is the one where the solution to Equation 3.22 reduces to a single real value. The solution to this problem is a quartic equation in terms of $t_{2}$ that is difficult to solve analytically. In practice however, one usually is presented with the problem of finding $j_{1}$ and $t_{1}$ for a desired valve travel time. Having picked the largest possible value of $j_{1}$, one would usually check whether the required acceleration at time $t=t_{1}$ does not violate the torque constraint. If the required acceleration is too high, the transition time is too short or the valve lift is too high.

Given that the initial displacement, velocity and acceleration are zero, and values for $j_{1}$ and $t_{1}$ from Equations 3.20 and 3.23, the acceleration $a$, velocity $v$, and displacement $s$ profiles can be obtained by integrating the jerk profile:

$$
\begin{aligned}
& a(t)=a\left(t_{i}\right)+\int_{t_{i}}^{t} j(t) d t \\
& v(t)=a\left(t_{i}\right)+\int_{t_{i}}^{t} a(t) d t \\
& s(t)=v\left(t_{i}\right)+\int_{t_{i}}^{t} v(t) d t
\end{aligned}
$$

The jerk profile in Figure 3.2 can be written as:

$$
j(\tau)= \begin{cases}j_{1} & 0 \leq t<t_{1} \\ j_{2} & t_{1} \leq t<t_{3} \\ j_{1} & t_{3} \leq t<t_{4}\end{cases}
$$

where $j_{1}, j_{2}$ are the magnitudes of jerk in different regions. Integrating Equation 3.25 with respect to time, and denoting the maximum acceleration 
and deceleration magnitudes with $a_{1}$ and $a_{2}$ respectively, the acceleration profile can be expressed as:

$$
a(t)= \begin{cases}j_{1}\left(t-t_{1}\right) & 0 \leq t<t_{1} \\ a_{1}+j_{2}\left(t-t_{2}\right) & t_{1} \leq t<t_{2} \\ j_{2}\left(t-t_{3}\right) & t_{2} \leq t<t_{3} \\ a_{2}+j_{1}\left(t-t_{4}\right) & t_{3} \leq t<t_{4}\end{cases}
$$

The same idea can be applied to the velocity and position profiles. For constant jerk, acceleration profiles are linear, velocity profiles are parabolic and displacement profiles are cubic. The advantage of using a jerk limited profile is that triangular acceleration profiled trajectories have smoother velocity, acceleration and jerk characteristics compared to other profiled trajectories. The control signals resulting from the utilization of such a reference trajectory will also be smoother, hence reducing the risk of impacting the drives, or exciting the machine's structural dynamics.

In the simulation of FFVA system, a Waveform Generator is programmed to generate the reference trajectory. The transition timing and the displacement of the valve can be set.

\subsection{Design Example}

From the sections above, two important criteria for motor selection were derived which are summarized as follows:

- $a_{\max }=\frac{T_{\max }}{2 \sqrt{J_{m} m}}$

- $E \propto \frac{J_{m} R}{K_{T}^{2}}$

The first criteria shows the maximum acceleration is constraint by two motor parameters: The maximum torque, and the inertia of the motor. The energy consumption for a fixed trajectory and a fixed valve mass provides the second rule to guide the motor selection process.

In addition, energy consumption for a desired transition time with a suboptimal triangular trajectory can also be compared using Equation 3.21. 


\subsection{Design Example}

\begin{tabular}{|c|c|c|c|}
\hline \multicolumn{4}{|c|}{ Comparison with other proposed models } \\
\hline Motor Name & QB02302 & 119007 & $\mathrm{I} 2383092 \mathrm{NC}$ \\
\hline Manufacturer & Maccon[27] & Pittman[33] & MCG[34] \\
\hline Torque Constant $K_{T}[\mathrm{Nm} / \mathrm{A}]$ & 0.076 & 0.0718 & 0.066 \\
\hline Terminal Resistance $R[\Omega]$ & 0.24 & 0.541 & 1.12 \\
\hline Motor Inertia $J_{m}\left[\mathrm{~kg} \cdot \mathrm{m}^{2}\right]$ & $2.3 \mathrm{E}-5$ & $3.1 \mathrm{E}-5$ & $1.7 \mathrm{E}-5$ \\
\hline Peak Torque $T_{\max }[\mathrm{Nm}]$ & 7.6 & 2.19 & 1.235 \\
\hline Acceleration Term $\frac{T_{\max }}{\sqrt{J_{m}}}$ & 1585 & 393 & 299 \\
\hline Energy Cost Term $\frac{J_{m R}^{m}}{K_{T}^{2}}$ & $9.56 \mathrm{E}-6$ & $32.5 \mathrm{E}-6$ & 43.7E-6 \\
\hline $\begin{array}{l}\text { Energy Cost } E \\
\text { for } 6000 \text { rpm }[\mathrm{J} / \text { Cycle }]\end{array}$ & 2.68 & 9.12 & 12.3 \\
\hline
\end{tabular}

Table 3.1: Motor Selection

In Table 3.1, three candidates with similar size are listed. The size is chosen in order to provide the minimum inertia that fulfills the linearity constraint between valve movement and motor movement. Since the QB02302 series motor outperforms the other candidates in terms of energy consumption and acceleration, it is used in the experimental test bed in Chapter 7.

It should be pointed out that the QB02302 motor can be ordered with different windings.

To make a first approximation, changing the windings from $N$ to $N^{*}$ affects the motor parameters as follows:

$$
K_{T}^{*}=\frac{N^{*}}{N} K_{T}, R^{*}=\left(\frac{N^{*}}{N}\right)^{2} R, L^{*}=\left(\frac{N^{*}}{N}\right)^{2} L
$$

Note that the energy cost term is not affected by changing the number of windings. Also, the maximum torque and thus, the available acceleration does not change, even though the current required to achieve this torque will be altered, since the torque constant $K_{T}$ is linearly related to the number of windings:

$$
I_{\max }=\frac{N}{K_{T} N^{*}} T_{\max }
$$




\subsection{Design Example}

Another interesting relationship for the FFVA system is the jerk available for a given maximum supply voltage $U_{\max }$ :

$$
j=\frac{K_{T} r_{a}}{J L}\left(U_{\max } \frac{N}{N^{*}}-\frac{K_{T} v}{r_{a}}-R \frac{a J}{K_{T} r_{a}}\right)
$$

Equation 3.28 indicates that the available jerk scales linearly with voltage and the inverse of the winding ratio. In addition, the jerk is reduced once the valve starts moving. To summarize, decreasing the number of windings increases the available maximum jerk. However, the current required to achieve the maximum acceleration is increased at the same time. Selecting the optimum number of windings then depends on the available voltage, current, and the type of trajectory chosen to reach the required valve dynamics. For our trajectories, a higher initial jerk leads to lower energy consumption. It is expected then, that a lower winding number improves efficiency at the expense of higher currents.

For the FFVA system with a QB02302 motor, Figure 3.3 shows a plot of maximum current, maximum torque and energy consumption vs. winding ratio: This plot shows that fewer windings require moderately less energy and a slightly smaller maximum torque. However, these advantages are offset by a large increase in required current. Note that there is a minimum value for maximum current and the winding ratio chosen for the FFVA system is close to that minimum value.

Before presenting an implementation of a system with the stock QB02302 motor, the following chapter derives how to design a custom motor for this application. 


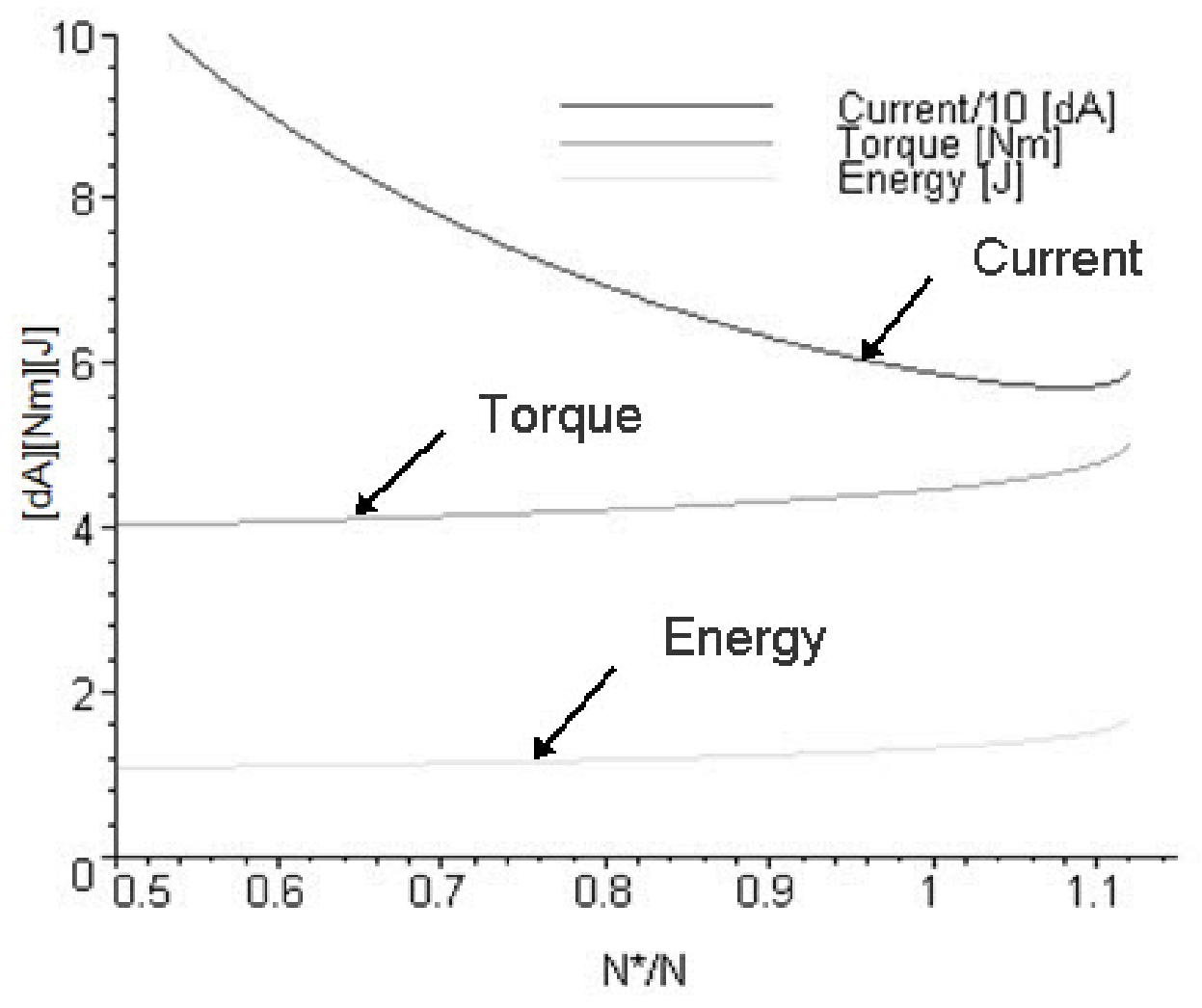

Figure 3.3: Winding variation for QB02302 


\section{Chapter 4}

\section{Motor Optimization}

The last chapter showed that motor parameters play an important role in the energy consumption and the acceleration capability of the FFVA system. Motor selection criteria were presented that can be used to choose the best off-the-shelf motor. However, in the FFVA system, the motor is used for a transient application, while the majority of motors on the market are designed for continuous application. Thus, off-the-shelf motors usually do not provide an optimal design for this application. This chapter then attempts to provide an analytical method to design an optimum motor. Several critical constraints should be considered when such a BLDC motor is designed. First it will be demonstrated that the stator saturation determines the thickness of the permanent magnets used on the rotor. Then a constraint on magnet demagnetization will provide a relationship between the acceleration of the actuator and the motor length. Thereafter, the need for a linear relationship between rotor movement and valve movement will be used to define radius of the rotor. Finally, it will be shown, that a relationship between rotor and the stator radius governs the energy consumption of the actuator.

\subsection{Stator Saturation Constraint}

The iron core of the stator consists of teeth and slots. The slots are filled with windings that create a stator field. The teeth are used to guide the stator field due to the current in the stator windings and the rotor field due to permanent magnets on the rotor. In order to ensure efficient use of the stator material, the stator core should not be saturated. This section shows that stator saturation can be used to determine an optimal thickness of the permanent magnets on the rotor.

A variety of magnet materials are available to provide the required rotating magnetic field in a brushless dc motor. The most popular are 
neodymium-iron-boron $(\mathrm{NdFeB})$ magnets, which are expensive but have the highest $B-H$ characteristic. The simplest form of brushless dc motor rotor construction uses a cylindrical shaft and a ferromagnetic rotating structure with a surface on which magnets are affixed. The magnets are radially magnetized and the magnet outer surfaces are ground or preformed to be concentric with the stator inside diameter. The magnets are held in place by a structural adhesive to prevent radial movement during operation. A magnetic field is generated by the magnets and it passes through the magnetic circuit formed by different parts of the motor.

The flux density in the airgap $B_{g o}$ due to the magnet alone and the acceptable linear current load $K_{s}$ are important factors in motor design.

In this section, we will show how to choose the best value of $B_{g o}$. This is also called selecting the operating point of the magnet. If a magnet with radial thickness $l_{m}$ is selected, the flux density in the airgap can be expressed by the magnet residual flux density $B_{r}$ :

$$
B_{g o}=\frac{l_{m}}{l_{g e}} B_{r}
$$

where $l_{g e}$ is the effective length of the airgap including the distance from the stator teeth to the rotor iron core. Usually the effect of slotting is also included. Approximately, $l_{g e}=l_{g}+l_{m}$.

Ideally speaking, reducing the airgap $l_{g}$ could obtain the same $B_{g o}$ by using less permanent magnet material. But in practice, the minimum value of the airgap is set by mechanical considerations. The usual range of $l_{g}$ is above $0.5 \mathrm{~mm}$.

Choosing the operating point of the magnet to be at the maximum energy product of the magnet material will result in minimum magnet volume and magnet cost [35]. However, the resultant average air gap flux density will be low (about one half of the magnet $B_{r}$ ), and therefore the armature winding will take a larger portion of the stator volume to provide the larger total current in order to generate the same torque.

Using a thicker magnet results in a more expensive magnet, but increases the airgap flux density, reduces the armature current loading, and results in a better balance of stator lamination iron and stator copper. But the flux density in the iron core $B_{c}$ should also be considered before deciding $B_{g o}$. The maximum flux density in the iron core is usually limited in order to avoid saturation. For the QB02302 motor, the material of the stator is 
"TRAFOPERM N3", whose saturation point is about $B_{s}=2.03 T$. In order to leave some margin, it is set conservatively to $1.8 T$, thus:

$$
B_{c}=\frac{w_{t}+w_{s}}{w_{t}} B_{g o}<1.8 T
$$

where $w_{s}$ is the width of each slot and $w_{t}$ the width of each tooth. For most machines (including our motors), the slotting of the stator is usually made $w_{t} \approx w_{s}$. Thus $B_{g o} \approx 0.9 T$ is a reasonable choice.

The next section shows how the demagnetization constraint affects the acceleration capabilities of the motor. This will lead to a discussion on how to choose the linear current load $K_{s}$.

\subsection{Demagnetization Constraint}

The performance of a BLDC motor used in transient application is mainly determined by the maximum acceleration it can provide, thus a high linear current loading is desirable. But the current density in the stator must be constrained not to demagnetize the permanent magnet. From a demagnetization curve, a critical point $\left(B_{D}, H_{D}\right)$ can be found, which should not be exceeded if demagnetization is to be avoided. With currently available $\mathrm{NdFeB}$ material, a value of about $B_{D}=-0.2 T$ can be achieved below a temperature of $120^{\circ} \mathrm{C}$.

In a BLDC motor, the flux density is distributed as in Figure 4.2. The first plot shows the geometry of a magnet on the rotor. $w_{p}$ is the width of each pole, $w_{m}$ is the span width of the magnet and $l_{m}$ is the thickness of the magnet. The second plot shows $B_{I}$, which is the sinusoidally distributed flux density created by the stator current. The last plot adds the flux density due to the magnet alone, $B_{g o}$, and $B_{I}$ in order to obtain the total flux density along the airgap. Clearly, the flux density of any point on this curve should be kept above $B_{D}$. If $\beta$ is the magnet span in mechanical degrees, or simply defined as $\beta=w_{m} / w_{p}$, then:

$$
B_{I, p e a k} \sin \beta \leq B_{g o}-B_{D}
$$

Since the peak value of $B_{I}$ can be expressed as [36]:

$$
B_{I, p e a k}=\frac{2 \sqrt{2} r \mu_{0} K_{s}}{p l_{g e}}
$$




\subsection{Demagnetization Constraint}

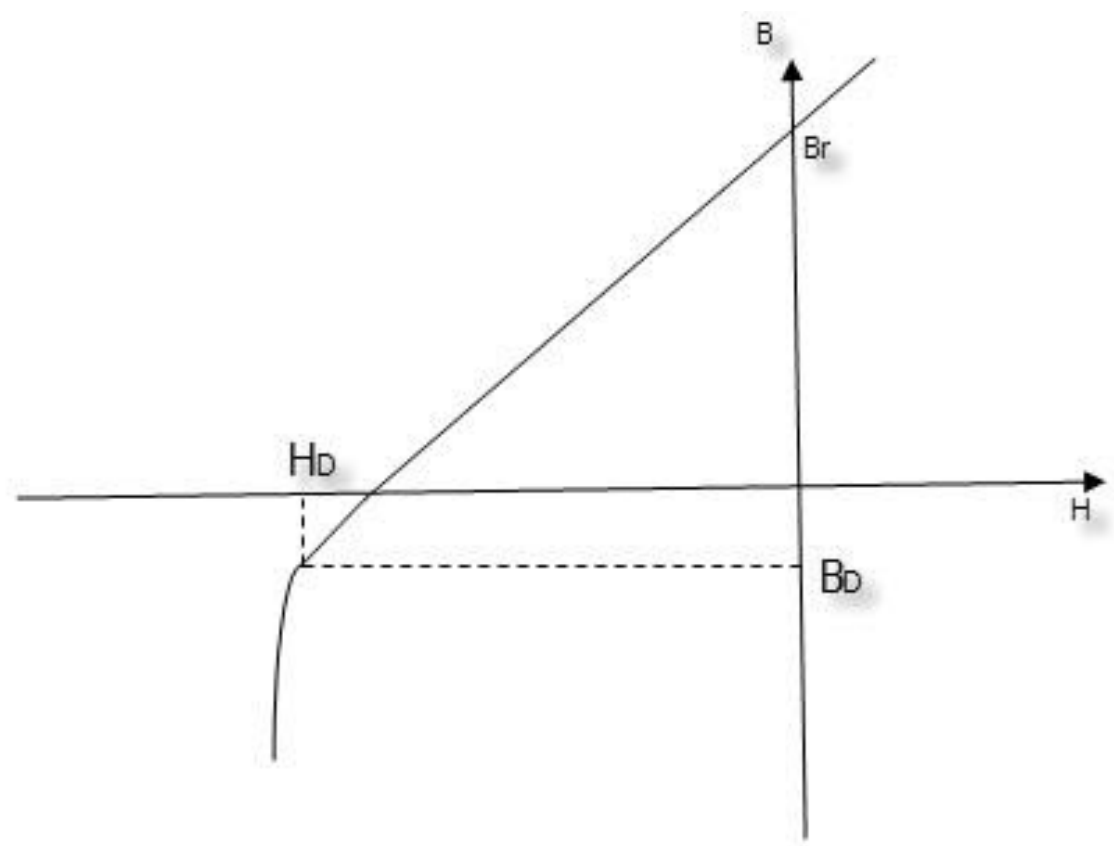

Figure 4.1: Demagnetization curve [36]

where $K_{s}$ is the rms linear current density along the stator periphery. Notation $p$ represents the number of poles. So the demagnetization constraint is given by [36]:

$$
K_{s} \leq \frac{p l_{g e}\left(B_{g o}-B_{D}\right)}{2 \sqrt{2} r \mu_{0} \sin \beta}
$$

For a PM motor, the torque can be expressed as [36]:

$$
T=2 \pi r^{2} l B_{g 1} K_{s} \sin \gamma
$$

where $\gamma$ is the angular displacement between the fields produced by the magnet and the stator current. The maximum value is found at $\sin \gamma=1$. $B_{g 1}$ is the rms value of the fundamental space component of the air gap flux density due to the magnet. It evaluates to

$$
B_{g 1}=\frac{2 \sqrt{2} B_{g o} \sin \beta}{\pi}
$$

Since we know the maximum linear current density $K_{s}$ (see Equation 


\subsection{Demagnetization Constraint}

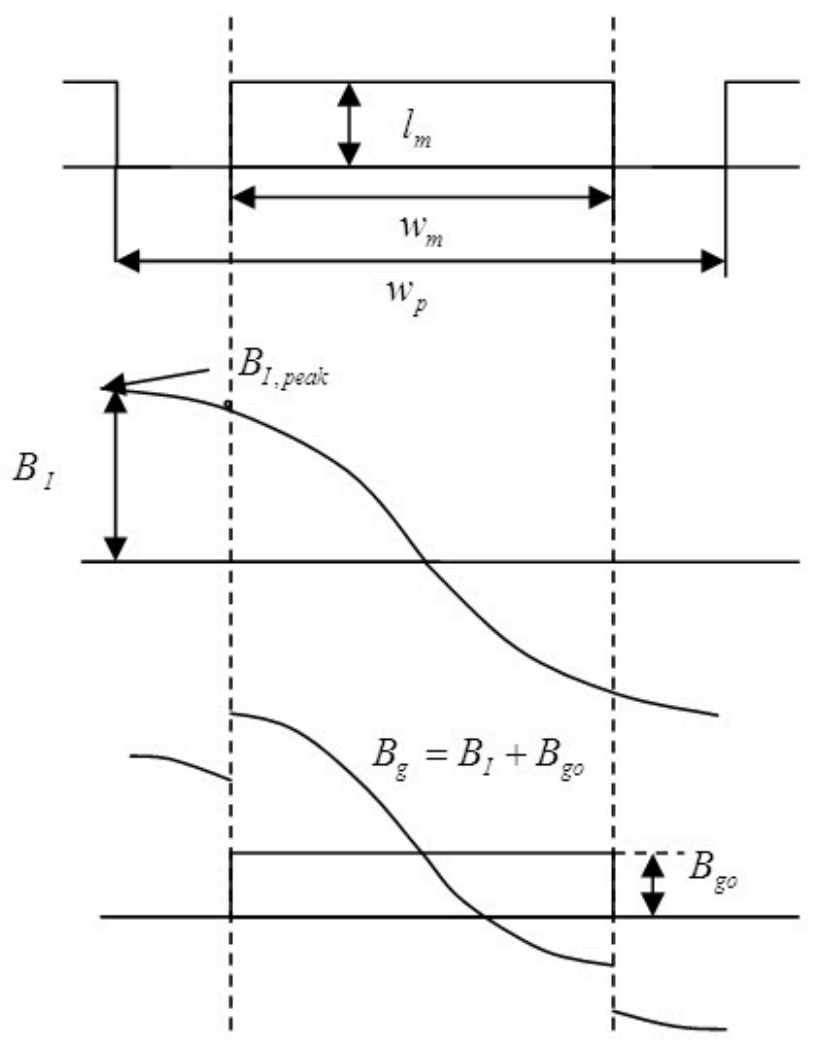

(1)

(2)

Figure 4.2: Flux density due to current and magnet [37]

4.5), then the maximum torque of a 3 -phase motor is given by:

$$
T_{\max }=\frac{2 r l p l_{m} B_{r}\left(B_{g o}-B_{D}\right)}{\mu_{0}}
$$

In practice the stator length $l$ should be an effective length, thus a length factor $k_{w}$ (since there are windings stretching out of the stator frame at both ends) should be added. Then the equation above turns into [38]:

$$
T_{\max }=\frac{2 r l p l_{m} B_{r}\left(B_{g o}-B_{D}\right)}{\mu_{0}} k_{w}
$$

This equation does not include saturation of the iron core. Especially for small motors, this can lead to substantial errors. Substituting Equation 4.9 
and 3.12 into 3.14 provides an expression for maximum valve acceleration in terms of geometric dimensions and material properties (the expression of $J_{m}$ can be found in next section) of the motor [38]:

$$
a_{\max }=\frac{T_{\max } r_{a}}{2 J_{m}}=\frac{\sqrt{2} p l_{m} B_{r}\left(B_{g o}-B_{D}\right) k_{w}}{\mu_{0} \sqrt{\pi \rho m}} \frac{\sqrt{l}}{r}
$$

The equation above shows that the maximum acceleration is proportional to the number of pole pairs $p$ :

$$
p=\frac{2 \pi r}{3 q\left(w_{s}+w_{t}\right)}
$$

where $q$ is the slot number per pole per phase. For small motors, $q=0.5$ is often used, because it provides a good torque to volume ratio. These motors are called fractional-slot winding motors [39]. In practice, the minimum tooth and slot widths are limited because of manufacturing constraints. Thus, $p$ is function of rotor radius $r$. Substituting Equation 4.11 into the equation above gives:

$$
a_{\max }=\frac{\sqrt{2} l_{m} B_{r}\left(B_{g}-B_{D}\right) k_{w}}{\mu_{0} \sqrt{\pi \rho m}} \frac{\sqrt{l}}{r} \frac{2 \pi r}{3 q\left(w_{s}+w_{t}\right)}=K_{\alpha} \sqrt{l}
$$

From the equation above, it can be seen that the maximum acceleration is proportional to $\sqrt{l}$. Thus, for high acceleration requirements, the rotor length should be chosen as large as possible, or if there is a constraint on motor length, then the maximum acceleration available can be estimated.

It needs to be pointed out that Equation 4.12 is based on a demagnetization constraint of the permanent magnets, rather than a saturation constraint of the iron core. Thus, this equation does not provide good quantitative information of the available acceleration, especially for small motors. It only provides the qualitative guideline that the acceleration is length dependent.The previous sections showed how to select the magnet thickness and the rotor length. In the upcoming section, criteria for rotor radius are provided.

\subsection{Linearity Constraint}

The mechanical optimizations shown in chapter 3 were based on the assumption that there is a linear relationship between valve and rotor motion. This 
assumption requires that the rotor rotation is small. In practice, one would usually constrain the rotor rotation to a maximum value of $\theta_{\max }=30^{\circ}$, thus the length of the excenter arm is also constrained:

$$
r_{a}=\sqrt{\frac{J_{m}}{m}} \geqslant \frac{s}{\theta_{\max }}
$$

This defines the lower limit for the rotor's inertia:

$$
J_{m} \geqslant \frac{s^{2} m}{\theta_{\max }^{2}}
$$

In practice, the density of the rotor core and the permanent magnets attached to it, are very similar. Thus, the rotor can be assumed to be a solid cylinder with density, $\rho$, motor length, $l$, and rotor radius, $r$. The rotor's inertia $J_{m}$ can be expressed as:

$$
J_{m}=\frac{1}{2} \pi r^{2} l \rho r^{2}=\frac{1}{2} \pi l \rho r^{4}
$$

Substituting Equation 4.13 into Equation 4.15 we find:

$$
J_{m}=\frac{1}{2} \pi l \rho r^{4} \geqslant \frac{s^{2} m^{2}}{\theta_{\max }^{2}}
$$

So,

$$
r^{4} l \geqslant \frac{2 s^{2} m^{2}}{\theta_{\max }^{2} \pi \rho}
$$

Since the length of the rotor has been determined by the acceleration requirement of the application, Equation 4.17 provides a constraint for the minimum rotor radius. At this point the rotor dimensions are fully defined by the valve acceleration requirements, the constraints on stator saturation, and linearity between motor and valve motion. The following section shows how the stator diameter determines the energy consumption of the actuation device.

\subsection{Energy Constraint}

In the FFVA system, the energy loss should be less than $2.5 \mathrm{~J} /$ cycle. When the motor is designed, the index function $\frac{J_{m} R}{K_{T}^{2}}$ should be taken into account in order to minimize the energy loss. 
From above, we know the expression of $J_{m}$ :

$$
J_{m}=\frac{1}{2} \pi l \rho r^{4}
$$

and the torque constant $K_{T}$ can be derived as [38]

$$
K_{T}=\frac{T}{I}=\frac{1}{3} \cdot\left(Z \cdot 2 r \cdot l \cdot B_{g o}\right) k_{w}
$$

The motor's terminal resistance $R$ can be expressed by a function of conductors, motor length, and copper area per slot, $A_{c a}$ :

$$
R=f\left(\frac{Z^{2} l}{A_{c a}}\right)
$$

Then the energy cost term of motor parameter can be rewritten as

$$
\frac{J_{m} R}{K_{T}^{2}}=\frac{\frac{1}{2} \pi l \rho r^{4} f\left(\frac{Z^{2} l}{A_{c a}}\right)}{\frac{1}{9} \cdot\left(Z \cdot 2 r \cdot l \cdot B_{g o} \cdot k_{w}\right)^{2}} \propto \frac{r^{2}}{A_{c a}},
$$

which shows that the cost term is proportional to $r^{2}$ and $\frac{1}{A_{c a}}$, but is independent of the stator length $l$ or number of conductors $Z$. If we want to reduce the energy loss, the radius of the rotor should be decreased or the copper area in each slot increased. The minimum radius is constrained by the linearity requirement from the previous section. The copper area is constrained by the slot area and the ability to fill the slot area with copper. The remainder of this section is then concerned with the relationship between stator geometry and copper area in each slot.

The copper area in each slot is related to the wire diameter, $d_{w}$, and the number of wires in each slot, $n_{w}$ :

$$
A_{c a}=n_{w} \pi\left(\frac{d_{w}}{2}\right)^{2}
$$

The stator slot cannot be completely filled with conductors, since there is always space left around the wires. The ratio of slot area, $A_{\text {slot }}$, to conductor area, $A_{c a}$, is defined as the filling factor, $k_{f i l l}$ :

$$
k_{f i l l}=\frac{A_{c a}}{A_{\text {slot }}}
$$


The slot area is related to the number of slots, $Q$, the stator inner radius, $r_{s i}$, the slot height, $h_{s}$, and the tooth width, $w_{t}$ :

$$
A_{s l o t} \approx \frac{\pi}{Q}\left[\left(r_{s i}+h_{s}\right)^{2}-r_{s i}^{2}\right]-w_{t} h_{s}
$$

Then the outer radius of the stator is found as:

$$
r_{s o}=r_{s i}+\left(h_{s}+h_{r s}\right)
$$

where $h_{r s}$ is the required thickness of the stator back. In Figure 2.4, it can be seen that the stator back must be larger than half the tooth thickness:

$$
h_{r s} \geq \frac{1}{2} w_{t}
$$

\subsection{Motor Design Example}

The DC motor (QB02302) chosen for the FFVA system has the parameters shown in Table 7.1. This is a very good motor, but it was not specifically designed for our application. This section then shows how to optimize the motor for the FFVA system.

1. Motor length $l$ : The acceleration capability of the FFVA system is primarily determined by the length of the motor (see Equation 4.12). For the stock motor, the maximum valve acceleration can be determined as: $a_{\max }=\frac{T_{\max } r_{a}}{2 J_{m}}=6300 \mathrm{~m} / \mathrm{s}^{2}$. The ideal triangular trajectory shown in Section 3.3 for a lift of $8 \mathrm{~mm}$ and an engine speed of $6000 \mathrm{rpm}$ is approximately $2500 \mathrm{~m} / \mathrm{s}^{2}$. Clearly, the stock motor can provide higher accelerations than necessary. By reducing the motor length to one half of its original length, the maximum possible acceleration reduces to $4400 \mathrm{~m} / \mathrm{s}^{2}$. This is still more than the ideal triangular trajectory prescribes and it leaves room for the suboptimal performance to introduce more inductances.

2. Rotor Radius $r$ : In the last step, the length of the motor was decreased, thus the rotor inertia $J_{m}$ becomes smaller. The linearity constraint needs to be verified. Equation 4.17 can be rewritten as:

$$
r^{4} \geqslant \frac{2 s^{2} m^{2}}{\theta_{\max }^{2} \pi \rho l}
$$


For lift $s=8 \mathrm{~mm}$, valve's mass $m \approx 40 \mathrm{~g}$, motor length $l=28 \mathrm{~mm}$ the density of steel $\rho=7800 \mathrm{~kg} / \mathrm{m}^{3}$, and $\theta_{\max }=30^{\circ}$, the limit of $r$ is given by:

$$
r \geqslant 12.8 m m
$$

The rotor radius is kept at $14 \mathrm{~mm}$, still fulfills the linearity constraint.

3. Slot Area $A_{\text {slot }}$ : The two steps above are used to determine the rotor size. Energy consumption determines the stator size. It was shown in Section 3.4 that the energy consumption of the stock system is expected to be at least $7 \%$ larger than required. Thus, the goal of this step is to reduce the cost term $\frac{J_{m} R}{K_{T}^{2}}$ of the optimized motor to half of the value of the original QB02302 motor. The energy cost term has not been affected by the change in rotor length and the parameters left for us to manipulate are mainly the slot height $h_{s}$ and wire diameter $d_{w}$. The number of conductors will be kept. Thus, since the rotor length was cut in half, the inductance of the motor should also be halved. This should allow for more energy efficient trajectories.

(a) First, the slot area and related parameters for the original motor QB02302 are determined. The measured parameters are slot width $w_{s}=5.1 \mathrm{~mm}$, tooth width $w_{t}=5.0 \mathrm{~mm}$, number of wires per slot $n_{w}=72$, stator inner radius $r_{s i}=14.5 \mathrm{~mm}$, number of slots $Q=9$, airgap $l_{g}=0.5 \mathrm{~mm}$, thickness of stator back $h_{r s}=4.65 \mathrm{~mm}$. wire diameter $d_{w}=0.723 \mathrm{~mm}$ [40] (Gauge 21: constant maximum current is about $9 A$, transient maximum current is usually $6 \sim 7$ times of that, which is around $50 A$; the resistance is $42.00 \mathrm{hm} / \mathrm{km}$ ) and slot height $h_{s}=8.5 \mathrm{~mm}$.

According to Equation 4.22 the copper area of one slot is given by:

$$
A_{c a}=n_{w} \pi\left(\frac{d_{w}}{2}\right)^{2}=29.6 \mathrm{~mm}^{2}
$$

Thus, Equation 4.24 provides the expression for the slot area:

$$
A_{\text {slot }}=\frac{\pi}{Q}\left[\left(r_{s i}+h_{s}\right)^{2}-r_{s i}^{2}\right]-w_{t} h_{s}=68.8 m^{2}
$$

The fill factor can be calculated by using Equation 4.23:

$$
k_{f i l l}=\frac{A_{c a}}{A_{\text {slot }}}=43 \%
$$


Then outer radius of the stator is found as:

$$
r_{s o}=r_{s i}+\left(h_{s}+h_{r s}\right)=27.65 m m
$$

Additionally, the terminal resistance of the motor can be estimated by:

$$
R=\frac{2}{3} \times 42.0 O h m / k m \times Z l \approx 0.254 O h m
$$

The first constant term $\frac{2}{3}$ was applied because of the relation between the total terminal resistance (or line resistance) and phase resistance in the motor. It can be seen that the estimation is fairly close to the measured value given in Table 7.1, which is $0.240 h m$.

(b) In the next step, the wire diameter and the slot height are adjusted in order to provide for lower energy consumption. The slot height and the wire diameter are set to new values: $h_{s}=14 \mathrm{~mm}$, $d_{w}=1.15 \mathrm{~mm}$ (Gauge 17: constant maximum current is about $19 A$, transient maximum current is usually $6 \sim 7$ times of that, which is around $120 \mathrm{~A}$; the resistance is $16.60 \mathrm{hm} / \mathrm{km}$ ). Now following the same calculation procedure.

$$
\begin{gathered}
A_{c a}=74.8 \mathrm{~mm}^{2} \\
A_{\text {slot }}=140 \mathrm{~mm}^{2} \\
k_{\text {fill }}=53 \% \\
r_{\text {so }}=35 \mathrm{~mm}
\end{gathered}
$$

Thus, the copper cross section area and slot area have both increased. The filling factor becomes a little higher, but it is still in a reasonable range. The outer radius has increased by $7.35 \mathrm{~mm}$. Most importantly, the cost term $\frac{J_{m} R}{K_{T}^{2}}$ has decreased. From Equation 4.21, we know the copper loss of the system for an FFVA system with constant rotor radius is inversely proportional to the copper area:

$$
E \propto \frac{J_{m} R}{K_{T}^{2}} \propto \frac{1}{A_{c a}}
$$

Which means theoretically, the copper loss now is $\frac{29.6 \mathrm{~mm}^{2}}{74.8 \mathrm{~mm}^{2}}=$ $39.6 \%$ of the value that QB02302 consumes. 
Also, the terminal resistance of the motor can be estimated by

$$
R=\frac{2}{3} \times 16.6 \mathrm{Ohm} / \mathrm{km} \times \mathrm{Zl} \approx 0.050 \mathrm{Ohm} .
$$

However, the end effect of winding becomes more dominant. Since the motor length is reduced, the portion of noneffective winding which is stretching out of the stator increases, which means the actual value of terminal resistance will be bigger than the calculation.

In this chapter, major constraints of designing an optimized motor for the FFVA system were studied and a design procedure was presented. The procedure is systemically shown in the Figure 4.3 .

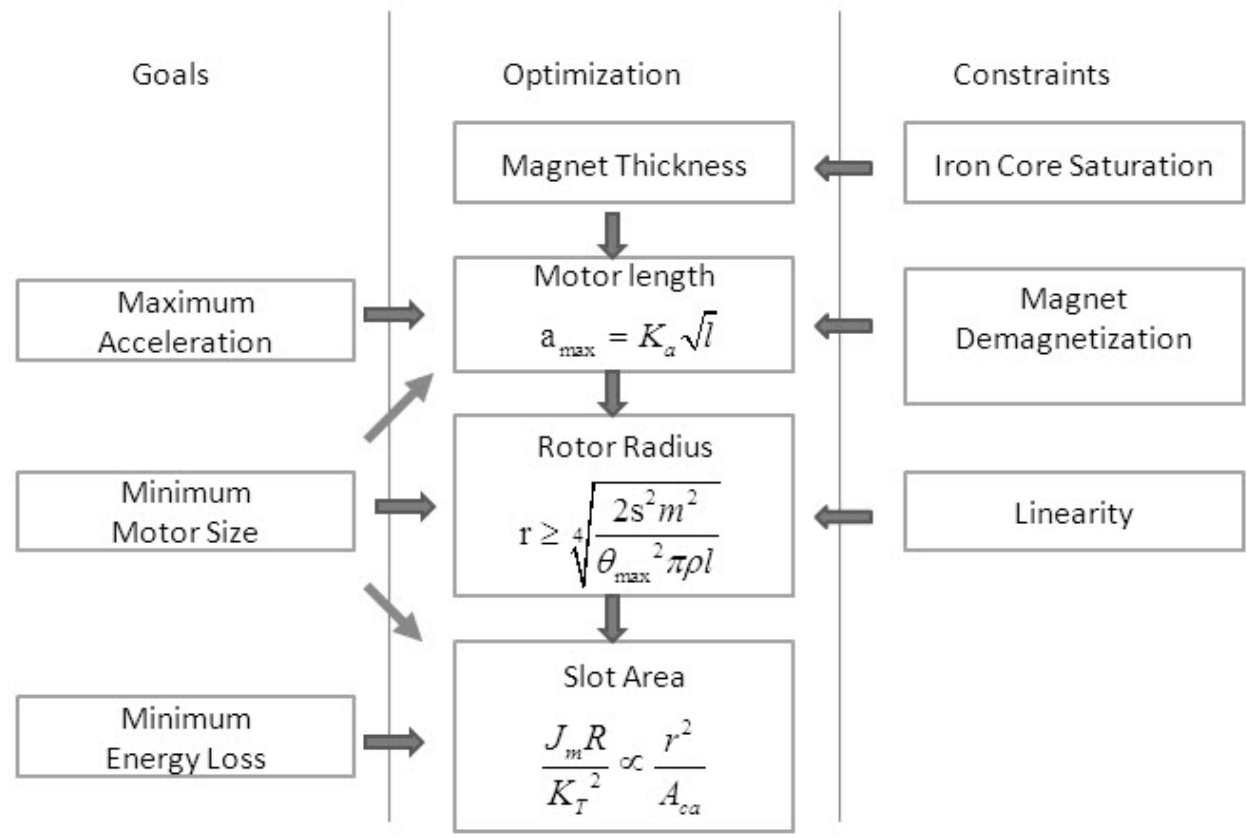

Figure 4.3: Diagram of optimization procedure

The derivation was verified by numerical calculation of the QB02302 parameters. Finally, the parameters of the QB02302 motor were optimized using approximate analytical expressions in order to provide better performance and a shorter motor. In the next chapter, the predicted performance of the optimized motor will be verified using finite element calculations. 


\section{Chapter 5}

\section{Finite Element Analysis of DC Motor}

In the FFVA system, a brushless DC motor drives the mechanical part and its copper loss is responsible for most of the energy losses of the actuation system. In a conventional internal combustion engine, the energy loss for each intake valve is about $2.5 \mathrm{~J} /$ cycle. Right now, the experimental FFVA platform using a stock QB02302 motor has an energy loss of about $2.7 \mathrm{~J} /$ cycle. In order to make the system compare favorably with the conventional ones, the energy loss must be reduced. According to the theoretical derivation, the energy cost term $\frac{J_{m} R}{K_{T}^{2}}$ should be minimized by optimizing motor parameters. In the last chapter, an optimized motor was given by derivation and calculation. In this chapter, both the stock QB02302 motor and the optimized motor are simulated using the finite element analysis software Maxwell SV. Then the parameters of the two models are compared.

This chapter first introduces the different topologies of the motors. Then the theoretical background of the FEM calculation is briefly explained. Finally the results of simulating the two motors are presented.

\subsection{Brushless DC Motor Topology}

Because of the motor's symmetry along the axis of the shaft, a $2 \mathrm{D}$ model is sufficient to analyze the important properties, while the complexity of the calculations are reduced. The transverse sections are given in Figure 5.1 and Figure 5.2. The structures of both motors are also symmetrical by every $120^{\circ}$, only one third of the layout is simulated, which is sufficient and concise for the Finite Element Analysis. Comparing the two models, it can be seen that the rotor structure is kept the same and the stator structure is changed by varying the slot height and outer diameter of the stator. 


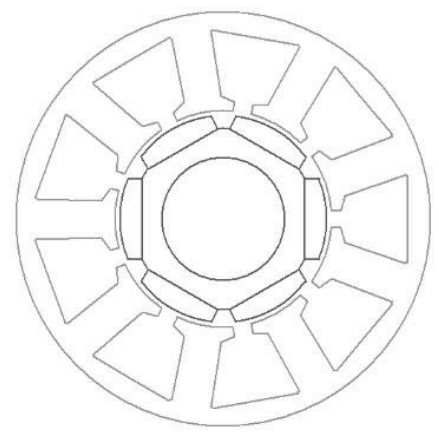

Figure 5.1: QB02302

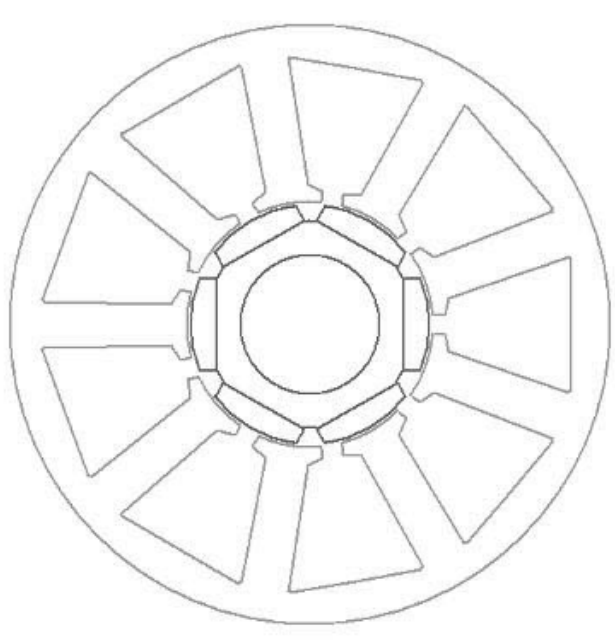

Figure 5.2: Optimized motor

\subsection{Maxwell Equations}

In electromagnetism, Maxwell's equations are a set of four partial differential equations that describe the properties of the electric and magnetic fields. Here, one of them is mainly used, which is called Ampere's circuital law:

$$
\oint_{L} \vec{H} d \vec{L}=\int_{S} \vec{J} d \vec{S}+\int_{S} \frac{d \vec{D}}{d t} d \vec{L}
$$

Here, $\vec{H}$ is the vector of the magnetizing field, $\vec{J}$ is the vector of the free current density and $\vec{D}$ is the vector of the electric displacement field. The constitutive equations are as follows:

$$
\begin{aligned}
& \vec{D}=\varepsilon \vec{E} \\
& \vec{B}=\mu \vec{H} \\
& \vec{J}=\sigma \vec{E}
\end{aligned}
$$

where $\vec{E}$ is the vector of the electric field, $\vec{B}$ is the vector of the magnetic field, $\varepsilon$ is the electric permeability, $\mu$ is the magnetic permeability and $\sigma$ is the electric conductivity. 


\subsection{Governing Equations in 2D Electromagnetic Field}

From the equations given above, 2D electromagnetic field governing equation [41] can be derived:

$$
\vec{\nabla} \times\left(\frac{1}{\mu} \vec{B}\right)=\overrightarrow{J_{t}}
$$

Or,

$$
\frac{\partial}{\partial x}\left(\frac{1}{\mu} \frac{\partial A_{x}}{\partial x}\right)+\frac{\partial}{\partial y}\left(\frac{1}{\mu} \frac{\partial A_{y}}{\partial y}\right)+J_{z}=0
$$

where $J_{t}$ is the total surface current while $J_{z}$ is the component of $J_{t}$ in the z-direction, and the flux density vector $\vec{B}$ is defined in terms of the potential vector $\vec{A}$ using the auxiliary equation:

$$
\vec{\nabla} \times \vec{A}=\vec{B}
$$

The boundary conditions are

$$
\left\{\begin{array}{l}
\Gamma_{t}: \frac{1}{\mu_{1}} \frac{\partial \overrightarrow{A_{1}}}{\vec{n}}+\frac{1}{\mu_{2}} \frac{\partial \overrightarrow{A_{2}}}{\vec{n}}=J_{s} \\
\Gamma_{u}: \vec{A}=\overrightarrow{A_{0}}
\end{array}\right.
$$

The natural boundary condition is represented by $\Gamma_{t}$ while the essential boundary condition is $\Gamma_{u}$. The equivalent surface current density $J_{s}$ equals the summation of change rate of the potential vector along the normal vector to the interface of the two fields, while the subscript refers to different parts on each side of the boundary. $\vec{n}$ is the normal unit vector. The potential vector on the border equals to the prescribed value. As long as we have a strong form of the governing equations for the $2 \mathrm{D}$ electromagnetic field, the weak form can be derived by using the minimal potential energy principle or the Galerkin method. Then, the weak form can be applied to each meshed element to obtain the stiffness matrix and the damping matrix.

So far, the general equations for a 2D electromagnetic field analysis have been defined. But for different parts of the BLDC motor, the forms of the equations are different. 
In the laminated stator, the iron core and air gap are

$$
\left\{\begin{array}{l}
\Omega_{1}: \frac{\partial}{\partial x}\left(\frac{1}{\mu} \frac{\partial A_{x}}{\partial x}\right)+\frac{\partial}{\partial y}\left(\frac{1}{\mu} \frac{\partial A_{y}}{\partial y}\right)=0 \\
\Gamma_{u}: \vec{A}=0
\end{array}\right.
$$

Here, $\Omega_{1}$ represents the field in the stator. $J_{z}$ becomes 0 since there is no free current going through the core and the eddy current can be omitted since the core is formed by stacked silicon steel. Beyond the inner and outer boundary of the core, the potential vector is 0 .

In the rotor iron core,

$$
\left\{\begin{array}{l}
\Omega_{2}: \frac{\partial}{\partial x}\left(\frac{1}{\mu} \frac{\partial A_{x}}{\partial x}\right)+\frac{\partial}{\partial y}\left(\frac{1}{\mu} \frac{\partial A_{y}}{\partial y}\right)=\sigma \frac{\partial A}{\partial t} \\
\Gamma_{t 1}: \frac{1}{\mu_{\text {core }}} \frac{\partial \overrightarrow{A_{\text {core }}}}{\vec{n}}+\frac{1}{\mu_{\text {air }}} \frac{\partial \overrightarrow{A_{\text {air }}}}{\vec{n}}=J_{s 1}
\end{array}\right.
$$

The term $\sigma \frac{\partial A}{\partial t}$ exists in the solid rotor iron core where eddy currents cannot be ignored.

There are two models which are commonly used to represent permanent magnets: the magnetization vector method and the equivalent current sheet method. The magnetizing vector method is used in this model for the permanent magnets:

$$
\left\{\begin{array}{l}
\Omega_{3}: \frac{\partial}{\partial x}\left(\frac{1}{\mu} \frac{\partial A_{x}}{\partial x}\right)+\frac{\partial}{\partial y}\left(\frac{1}{\mu} \frac{\partial A_{y}}{\partial y}\right)=\nabla \times\left(\frac{1}{\mu} B_{r}\right) \\
\Gamma_{t 2}: \frac{1}{\mu_{P M}} \frac{\partial \overrightarrow{A_{P M}}}{\vec{n}}+\frac{1}{\mu_{\text {air }}} \frac{\partial \overrightarrow{A_{\text {air }}}}{\vec{n}}=J_{s 2}
\end{array}\right.
$$

Here, the free current density is not 0 anymore, because there is induced current in the permanent magnet when the magnetic field is changing. The linear current density between air and the permanent magnet gives the natural boundary condition. 
In the windings, the following conditions hold:

$$
\left\{\begin{array}{l}
\Omega_{4}: \frac{\partial}{\partial x}\left(\frac{1}{\mu} \frac{\partial A_{x}}{\partial x}\right)+\frac{\partial}{\partial y}\left(\frac{1}{\mu} \frac{\partial A_{y}}{\partial y}\right)=-\frac{N I}{S} \\
\Omega_{5}: \frac{\partial}{\partial x}\left(\frac{1}{\mu} \frac{\partial A_{x}}{\partial x}\right)+\frac{\partial}{\partial y}\left(\frac{1}{\mu} \frac{\partial A_{y}}{\partial y}\right)=\frac{N I}{S} \\
U=R I+L \frac{d i}{d t}+\frac{N L}{S}\left(-\int_{n} \frac{\partial A}{\partial t} d \Omega_{n}+\int_{p} \frac{\partial A}{\partial t} d \Omega_{p}\right)
\end{array}\right.
$$

Since there is free current going through the windings in the positive or the negative direction, the current density can be expressed as the total current over area. The third equation is the coupling of the field and the external drive circuit. The boundary conditions were already given in the previous three parts.

These are governing equations for different parts of motors. They are not directly solved by the FEA software, because they are all partial differential equations. They have to be transferred to a weak form, which then can be solved numerically by the software.

\subsection{Finite Element Simulation for BLDC Motor}

The previous sections described the motor geometry, the governing equations, and the boundary conditions of the motor. These models are implemented in Maxwell SV using Galerkin's method for 3-node elements. This section describes the meshing process, the Galerkin's formulations, and typical plots of flux density and current density that are obtained with Maxwell SV.

Mesh generation for the FEM should be simple and robust, and the rotor mesh should be allowed to rotate easily. In this approach, the FEM mesh of the cross section of the BLDC motor is divided into three parts: the stator, the rotor and the magnet, with each including a part of the air gap. When the rotor is rotated according to the time step, the shape of the mesh for both the stator and rotor can be kept constant and only the coordinates of the rotor mesh and the periodic boundary condition on the interface need to change. Therefore, in this approach the stator mesh and the rotor mesh are required only need to be generated once. This can greatly reduce the computing time required to generate the FEM mesh at each time step. A 


\subsection{Finite Element Simulation for BLDC Motor}

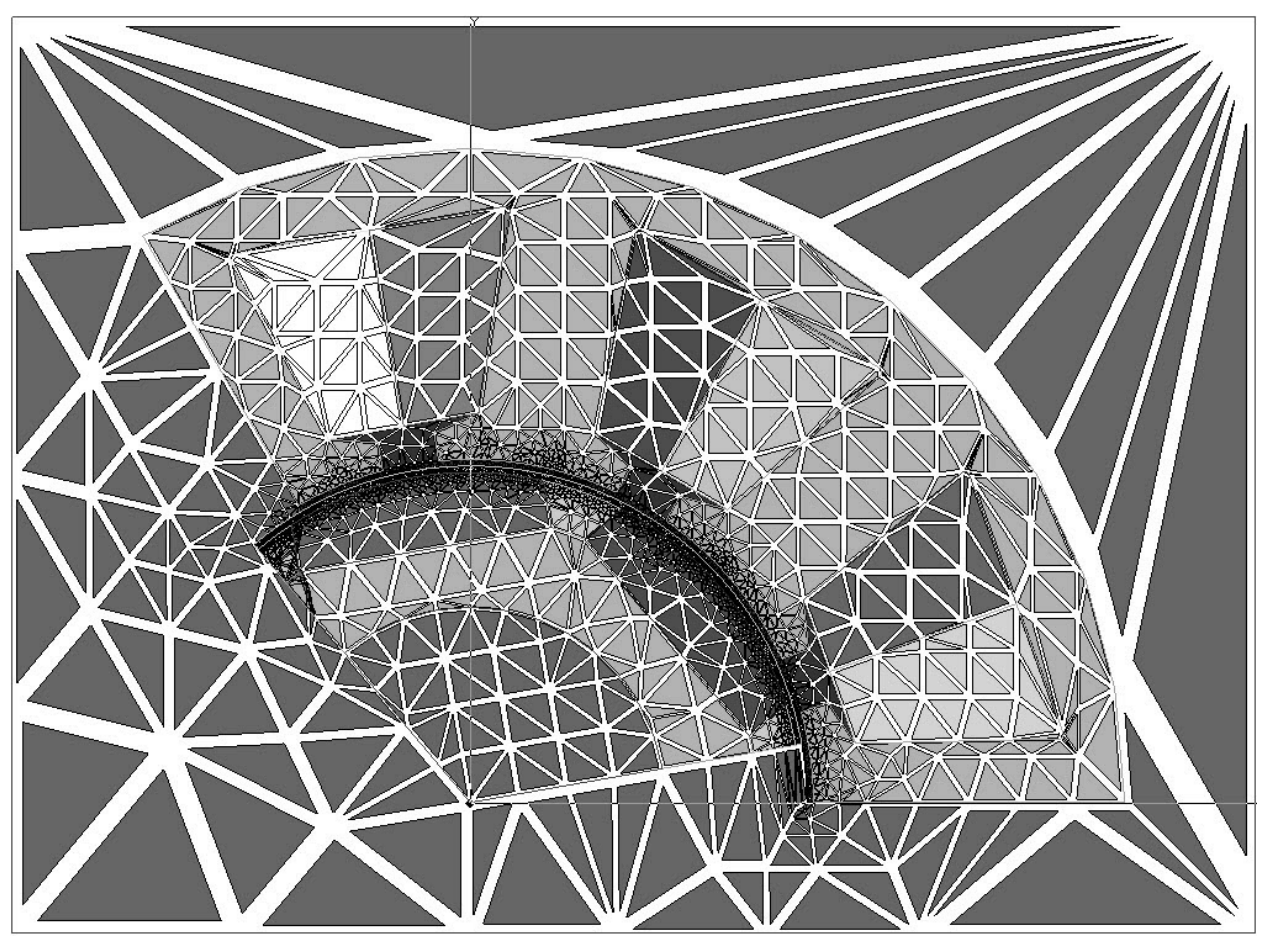

Figure 5.3: Mesh result

typical mesh for the stock QB02302 motor is shown in Figure 5.3. It is automatically generated by the software, but a finer mesh along the air gap was specified manually, because this is the most important part of the flux distribution. Additionally, the software checks for convergence and suggests finer meshes, if the convergence criteria are not met.

Galerkin's method is usually employed for the finite element formulation. This method uses particular weighted residuals for both the weighting functions and the shape functions.

According to the Galerkin's method, for a 3-node triangular element, the magnetic vector potential can be expressed as [42]

$$
A=\sum_{i=1}^{3} N_{i} A_{i}
$$

Here $A$ is the function of the vector potential, $N_{i}$ is the element shape 


\subsection{Finite Element Simulation for BLDC Motor}

function and the $A_{i}$ is the approximation of the vector potential at the nodes of the element.

The Galerkins formulation of the laminated stator iron core and air gap

$$
\iint\left(\frac{\partial N_{i}}{\partial x} \frac{\partial}{\partial x} \frac{1}{\mu} \sum_{j=1}^{3} N_{j} A_{j}+\frac{\partial N_{i}}{\partial y} \frac{\partial}{\partial y} \frac{1}{\mu} \sum_{j=1}^{3} N_{j} A_{j}\right) d x d y=0
$$

In matrix form, this turns into

$$
\left[\frac{1}{\mu}[G]\{A\}\right]=0
$$

For the rotor iron core, the formulation is

$\iint\left(\frac{\partial N_{i}}{\partial x} \frac{\partial}{\partial x} \frac{1}{\mu} \sum_{j=1}^{3} N_{j} A_{j}+\frac{\partial N_{i}}{\partial y} \frac{\partial}{\partial y} \frac{1}{\mu} \sum_{j=1}^{3} N_{j} A_{j}+\sigma N_{i} \frac{\partial A_{i}}{\partial t}\right) d x d y=0$

In matrix form, this can be written as

$$
\left[\frac{1}{\mu}[G]\{A\}+\sigma[T] \frac{\partial A}{\partial t}\right]=0
$$

For the permanent magnet, the formulation can be expressed as

$\iint \frac{1}{\mu}\left(\frac{\partial A}{\partial x} \frac{\partial N_{i}}{\partial x}+\frac{\partial A}{\partial y} \frac{\partial N_{i}}{\partial y}\right) d x d y=\iint \frac{\mu_{0}}{\mu}\left(M_{x} \frac{\partial N_{i}}{\partial y}-M_{y} \frac{\partial N_{i}}{\partial x}\right) d x d y$

In matrix form

$$
[G] A=B_{r x}\left[c_{i}\right]-B_{r y}\left[b_{i}\right]
$$

For the winding, the equation is

$$
\iint\left(\frac{\partial N_{i}}{\partial x} \frac{\partial}{\partial x} \frac{1}{\mu} \sum_{j=1}^{3} N_{j} A_{j}+\frac{\partial N_{i}}{\partial y} \frac{\partial}{\partial y} \frac{1}{\mu} \sum_{j=1}^{3} N_{j} A_{j}+N_{i} \frac{N I}{S}\right) d x d y=0
$$

Again, the equation can be rewritten in matrix form:

$$
\left[\frac{1}{\mu}[G]\{A\}+\{Q\} \frac{N I}{S}\right]=0
$$


For the circuit equation

$$
V=\frac{L}{S}\left[\int_{n} N_{i} \frac{\partial A}{\partial t} d \Omega_{n}-\int_{p} N_{i} \frac{\partial A}{\partial t} d \Omega_{p}\right]+R i+L \frac{d i}{d t}
$$

In matrix form:

$$
V=\frac{L}{S}\left[\left(\{Q\}\left\{\frac{\partial A}{\partial t}\right\}\right)_{n}-\left(\{Q\}\left\{\frac{\partial A}{\partial t}\right\}\right)_{p}\right]+R i+L \frac{d i}{d t}
$$

where,

$$
\begin{gathered}
T_{i j}=\iint N_{i} N_{j} d x d y= \begin{cases}\frac{\Delta_{e}}{6} & \text { if } i=j \\
\frac{\Delta_{e}}{12} & \text { if } i \neq j\end{cases} \\
Q=\iint N_{i} d x d y=\frac{\Delta_{e}}{3} \\
G=\iint\left(\frac{\partial N_{i}}{\partial x} \frac{\partial N_{j}}{\partial x}+\frac{\partial N_{i}}{\partial y} \frac{\partial N_{j}}{\partial y}\right) d x d y=\frac{b_{i} b_{j}+c_{i} c_{j}}{4 \Delta_{e}}
\end{gathered}
$$

and $\Delta_{e}$ is the triangular area of the element.

The field equations above and the circuit equation have to be solved simultaneously. Rotor movement has to be coupled. The coefficient matrix is symmetric.

The computed steady-state flux distributions when maximum current $100 \mathrm{~A}$ is applied in the stator winding is shown in Figure 5.4 (QB02302), which indicates that the iron core is not saturated even maximum current is applied. Forces and torques are calculated by integrating the Maxwell's stress tensors along a closed path in the air gap.

\subsection{Evaluation of Optimized Motor}

The simulations in the last section need to be compared to the analytical derivations from Chapter 4. The properties of the stock and the optimized motors are shown as in Table 5.1. The parameters of the optimized motor are given by both the FEM method and the analytical method. In the simulation, eddy currents and displacement currents are neglected. The permeability is linear. The iron core material is homogeneous. These assumptions are widely used in modeling DC and AC machines, permanent magnet devices, transformers and other electrical equipments [43]. In the table, it can be seen that the optimized motor has a smaller energy cost 
5.5. Evaluation of Optimized Motor

\begin{tabular}{|l||l|l|l|}
\hline Parameter & QB02302 & $\begin{array}{l}\text { Optimized } \\
(\mathrm{FEM})\end{array}$ & $\begin{array}{l}\text { Optimized } \\
\text { (Theory) }\end{array}$ \\
\hline Outer Radius of Stator $[\mathrm{mm}]$ & 27.65 & 35 & 35 \\
Length of stator core $[\mathrm{mm}]$ & 56 & 28 & 28 \\
Wire Diameter $[\mathrm{mm}]$ & 0.7229 & 1.15 & 1.15 \\
Torque Constant $K_{T}[\mathrm{Nm} / \mathrm{A}]$ & 0.076 & 0.042 & 0.036 \\
Terminal Resistance $R_{c}[\mathrm{Ohms}]$ & 0.24 & 0.067 & 0.050 \\
Motor Inertia $J_{m}\left[\mathrm{~kg} \cdot m^{2}\right]$ & $2.3 \mathrm{E}-5$ & $1.5 \mathrm{E}-5 *$ & $1.5 \mathrm{E}-5$ \\
Energy Cost Term $\frac{J_{m} R_{c}}{K_{T}^{2}}$ & $9.56 \mathrm{E}-6$ & $5.60 \mathrm{E}-6$ & $5.79 \mathrm{E}-6$ \\
\hline
\end{tabular}

Table 5.1: Comparison of motor parameters

term, which means that it consumes less energy for completing the same transition. Also, there is good agreement between the FEM simulation and the analytical expressions. The parameters of size are inherently the same since the models are identical. The difference of energy cost term between FEM and theory is mainly caused by two parameters: motor resistance and torque constant. The motor resistance given by the software includes the extra windings stretching out of the stator, which is not estimated in our theocratical calculation. The torque constant in the analytical calculation is an estimated value, with some assumptions, such as the flux density in the iron core is uniform and the magnet thickness is even. The FEM model is likely closer to the true motor parameters.

The value of the energy cost term in the optimized motor is almost half of that of the stock QB02302. Thus, the energy loss for the same operation is cut down to half in theory. After optimizing the geometry of the stator, the quantity of material used in the laminated core is increased. Even though the core loss has actually increased, it is relatively small comparing to the copper loss. It can be seen that the overall performance of the optimized motor is far better than the stock motor QB02302.

This chapter used a finite element simulation in order to validate the theoretical predictions of the optimized motor. This was achieved by simulating the original QB02302 motor and the new optimized motor. The QB02302 motor compared well with the specification sheet and the optimized motor compares well with the theoretical predictions from Chapter 4. The parameters obtained with the FEM model for the optimized motor will be used in Chapter 7 in order to simulate the overall actuation system. 


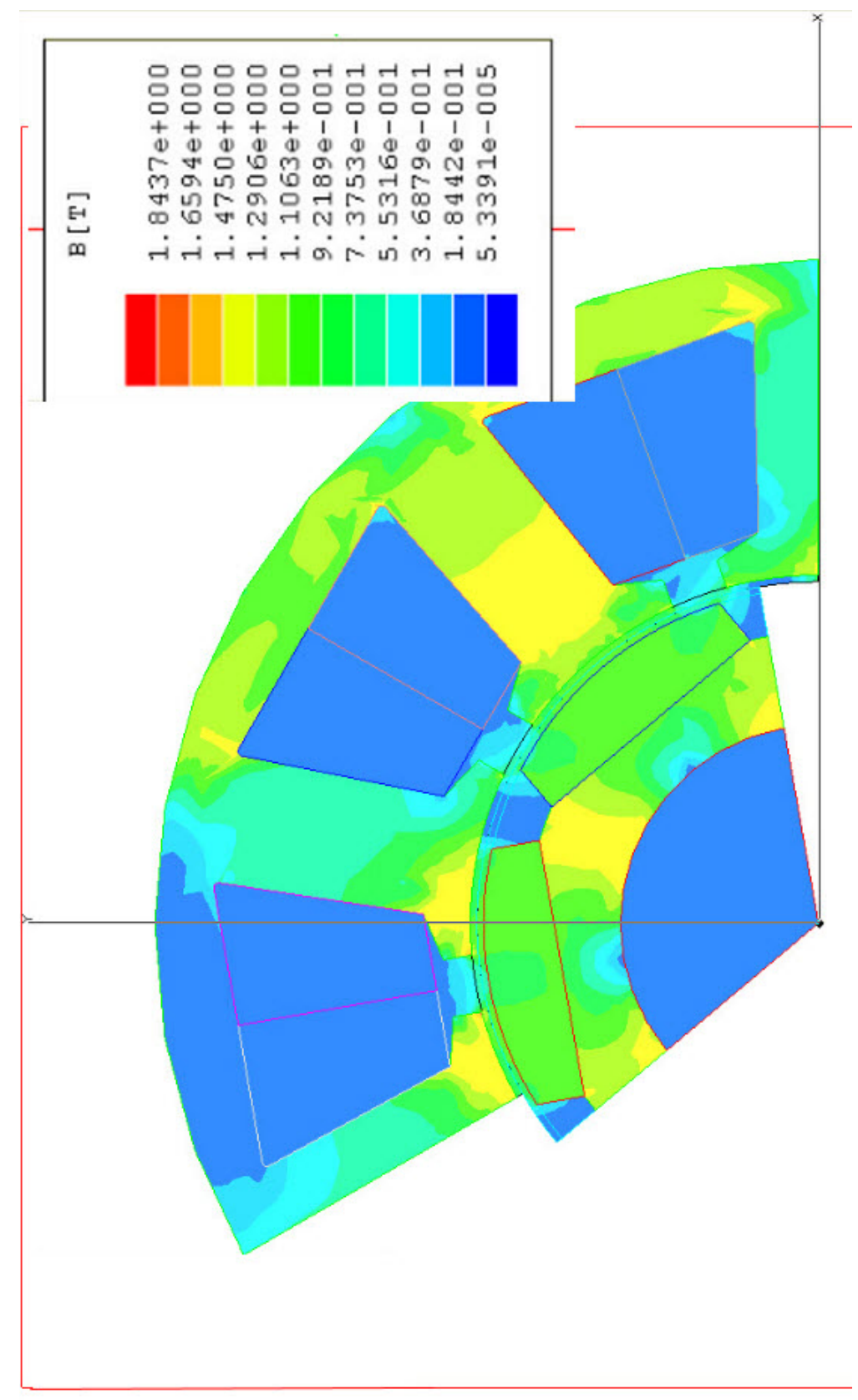

Figure 5.4: Flux density distribution of the optimized motor 


\section{Chapter 6}

\section{Control Strategy}

In this chapter, the technology for controlling the FFVA system is discussed. The main function of the control system is to move the valve from the closed to the opened position (and vice-versa) avoiding noise, which is caused by nonzero seating velocity. Similar to typical modern servo control systems, a cascaded control architecture is employed (see Figure 6.1). A conventional 3 phase inverter provides PI current control and commutation using measured values of position and current as well as reference current. The reference current is provided by a $\mathrm{PD}+$ Feedforward position controller that are implemented in a dSpace rapid prototyping system. The position controller receives its reference position from an energy minimized trajectory generator that is also implemented in dSpace. The algorithms for the trajectory generator have been provided in Chapter 3. Positions measurements are provided by an encoder. In order to minimize noise when deriving velocity from the position signal, a state observer is employed. A detailed description of the different parts of the controller follows in this chapter.

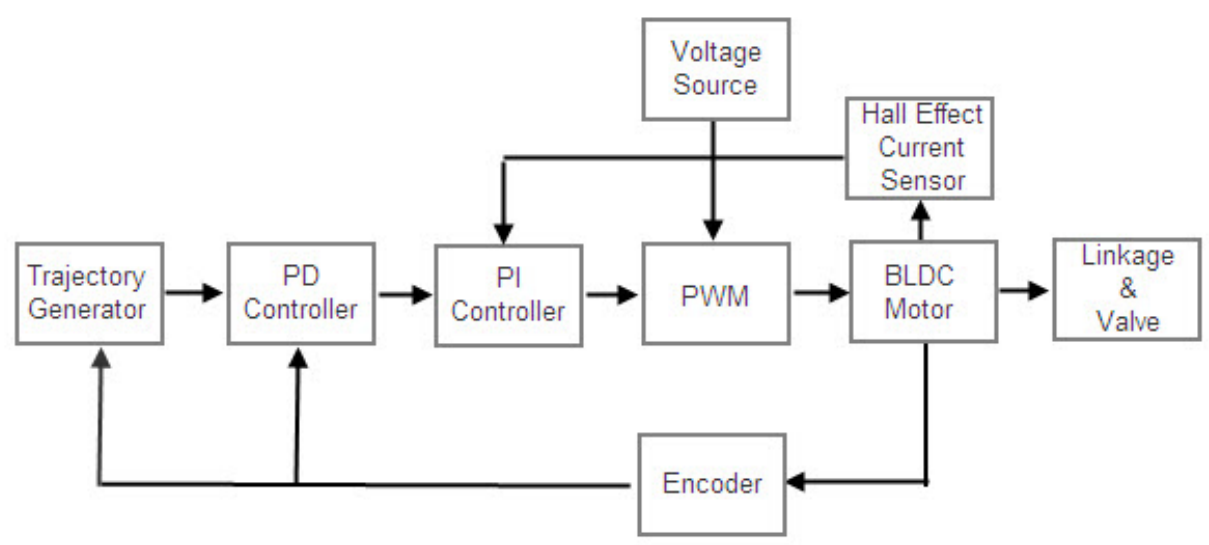

Figure 6.1: Diagram of control units 


\subsection{Phase Inverter}

The 3 phase inverter provides commutation and current control. Commutation is the process of switching current to generate a rotating magnetic field. In brushless systems, commutation is accomplished electronically using a rotor position feedback device. The rotor position sensors may be magnetic, optical, or any type of device that provides sufficiently accurate and reliable information on the motor position. In general, the best position for commutation is that point at which the back EMF waveform is "centered" between commutation points.

In addition to commutation, the 3 phase inverter also provides PI current control. Thus, to the outside the 3 phase inverter combined with the BLDC motor looks like a single phase DC motor plus inverter, because the 3 phase inverter takes on the function of the mechanical commutation device found in a conventional single phase DC motor. It is thus possible to model the 3 phase inverter and brushless DC motor as an equivalent single phase DC motor. A block diagram of this representation together with the PD position controller is shown in Figure 6.2. The position controller is built around the current controller. Usually in cascaded control loops, the inner current loop has a bandwidth $\omega_{i}$, that is considerably higher than the bandwidth $\omega_{p}$, of the outer position control loop. This allows independent tuning of the two control loops. Then, the current controller is shown in Figure 6.3:

Ignoring the back EMF due to motor speed, the transfer function of the current controller shown in Figure 6.3 can be written as

$$
\frac{I(s)}{I_{r e f}(s)}=\frac{P_{i} s+K_{i}}{L s^{2}+s\left(R+P_{i}\right)+K_{i}}=\frac{\frac{P_{i}}{L} s+\frac{K_{i}}{L}}{s^{2}+s\left(\frac{R+P_{i}}{L}\right)+\frac{K_{i}}{L}}
$$

The proportional gain $P_{i}$ and the integral gain $K_{i}$ of the PI controller can be derived from desired bandwidth of the current controller, $\omega_{i}$ (usually, $\zeta$ is close to 1$)$ :

$$
\omega_{i}^{2}=\frac{K_{i}}{L} ; \quad 2 \zeta \omega_{i}=\frac{P_{d}+R}{L}
$$

Then,

$$
P_{i}=2 \zeta \omega_{i} L-R ; \quad K_{i}=\omega_{i}^{2} L
$$

The value of the $\omega_{i}$ is usually selected to be approximately five times higher than the bandwidth of the position controller. 


\subsection{Position (or PD) Controller}

Position control can be implemented in a variety of different ways. Typically, Either a Lead lag controller or a PD controller are used for this task. In our case we have chosen a PD controller because it provides a simple strategy to set bandwidth and damping for the position loop. It also does quite well in rejecting disturbances and it fairly robust towards model uncertainties. Once the gains of the PI controller are determined, the current can be regulated fast and accurately. For the position controller, the current controller can be idealized as the unit gain block shown in Figure 6.4.

The transfer function ( $B_{m}$ can usually be neglected which reduces the complexity of the transfer function) for position control can be written as

$$
\frac{\theta(s)}{\theta_{r e f}(s)}=\frac{K_{d} s+P_{d}}{J s^{2}+K_{d} s+K_{d}}=\frac{\frac{K_{d}}{J} s+\frac{P_{d}}{J}}{s^{2}+\frac{K_{d}}{J} s+\frac{P_{d}}{J}}
$$

The proportional gain $P_{d}$ and the derivative gain $K_{d}$ of $\mathrm{PD}$ controller can be derived from the mechanical resonance frequency $\omega_{p}$ (usually, $\zeta$ is close to 1):

$$
\omega_{p}^{2}=\frac{P_{d}}{J} ; \quad 2 \zeta \omega_{p}=\frac{K_{d}}{J}
$$

Then,

$$
P_{d}=\omega_{p}^{2} J ; \quad K_{d}=2 \zeta \omega_{p} J
$$

The value of $\omega_{p}$ needs to be sufficiently high in order to allow tracking of the reference trajectory. For the FFVA system the trajectories at $6000 \mathrm{rpm}$ contain a fundamental frequency component at approximately $100 \mathrm{~Hz}$ with very few harmonics. Thus it is sufficient to set the bandwidth of the position controller to $350 \mathrm{~Hz}$ or $\omega_{p}=700 \times \pi \mathrm{rad} / \mathrm{sec}$.

\subsection{States Observer}

The PD position controller requires measured values of position and velocity. In commercial servo applications, resolvers or encoders are commonly used for measuring position. Velocity is either found by digitally differentiating 


\subsection{States Observer}

the position signal or by employing a tachometer. For the FFVA application in a real engine, encoders would not be suitable, because they are too expensive and cannot withstand the extended temperature range found in the cylinder head. Inductive pickups or hall sensors are much likelier solutions. However, in the test bed used in this thesis, an encoder is used, because it is readily available and provides excellent position resolution as well as high bandwidth.

The principal operation of the optical encoder is explained here. The encoder consists of light sources, a slotted disk, photo detectors, a bearing-unit and signal processing circuits. After the signal light passes through the slits on the the rotating disk, it is captured by photo detectors, and transduced to an electronic signal. There are two types of disks, an incremental and an absolute type disk. However, creating high resolution absolute encoders is more difficult and most encoders are of the relative type.

The encoder chosen for the FFVA test bed has a resolution of 20, 000 lines per revolution, which provides a highly accurate measurement of position. In Simulink, this encoder can be modeled by a "Quantizer" with a quantization interval of $2 \pi / 80000$.

The PD controller requires a position and a velocity measurement. To avoid additional hardware it is desirable to derive the velocity measurement from the position measurement. Even though the resolution of the direct position measurement is relatively high, the differentiation operator amplifies high frequency noise and reduces the resolution by the inverse of the sampling time:

$$
\begin{aligned}
& \text { resolution }(\omega)=\frac{\text { resolution }(\theta)}{\text { SamplingTime }} \\
& \text { resolution }(\alpha)=\frac{\text { resolution }(\omega)}{\text { SamplingTime }}
\end{aligned}
$$

Since the typical sampling time is $0.025 \mathrm{~ms}$, the resolution for velocity and acceleration using a simple backward difference operator deteriorates drastically. The quantization noise on the measured velocity is small for the encoder type chosen in the experimental setup. However, real sensors in actual engine applications would usually provide a much lower resolution that would then lead to significant quantization errors in the derived velocity measurements. A low pass filter (LPF) can be introduced to reduce this noise 


\subsection{States Observer}

and quantization errors, although it introduces a delay. The cutoff frequency of the LPFs should be high compared to the bandwidth of the position loop since this delay may deteriorate the performance of the control system.

In order to get better velocity estimation, a Kalman filter can be used. The specific Kalman filter designed for FFVA application is shown here.

The transfer function of a DC motor is generally described by

$$
\frac{\theta(s)}{e(s)}=\frac{\frac{K_{T}}{\left(J_{m} s+b_{m}\right)(L s+R)}}{1+K_{B} \frac{K_{T}}{\left(J_{m} s+b_{m}\right)(L s+R)}} \frac{1}{s}
$$

The numerical values for the parameters of the DC motor is given in Table 7.1.

When we design a discrete Kalman Filter for the system, discretization [44] should be done. The state vector is defined as:

$$
\mathbf{q}=\left[\begin{array}{l}
\theta \\
\alpha \\
\omega
\end{array}\right]
$$

If we set the sampling time at $0.25 e^{-4} m s$, and use the parameters given above, the discrete state space can be calculated:

$$
\begin{gathered}
\mathbf{A}=\left[\begin{array}{ccc}
1 & 0.001529 & 0 \\
0 & 0.9794 & 0 \\
0 & 121.9 & 0.9999
\end{array}\right] \quad \mathbf{B}=1.0 e^{-4}\left[\begin{array}{c}
0 \\
0 \\
0.001529
\end{array}\right] \\
\mathbf{C}=\left[\begin{array}{lll}
1 & 0 & 0
\end{array}\right] \mathbf{D}=0
\end{gathered}
$$

The predictor corrector type procedure of the Kalman filter can then be written as

$$
\begin{gathered}
\mathbf{q}(k)=\overline{\mathbf{q}}(k)+\mathbf{G}(k)[y(k)-\mathbf{C} \overline{\mathbf{q}}(k))] \\
\overline{\mathbf{q}}(k+1)=\mathbf{A} \mathbf{q}(k)+\mathbf{B u}(k) \\
\mathbf{G}(k)=\mathbf{M}(k) \mathbf{C}^{\mathbf{T}}\left[\mathbf{C M}(k) \mathbf{C}^{\mathbf{T}}+\mathbf{R}_{\mathbf{v}}\right]^{-1} \\
\mathbf{P}(k)=\mathbf{M}(k)-\mathbf{G}(k) \mathbf{C M}(k) \\
\mathbf{M}(k+1)=\mathbf{A} \mathbf{P}(k) \mathbf{A}^{\mathbf{T}}+\mathbf{R}_{\mathbf{w}}
\end{gathered}
$$

where $\overline{\mathbf{q}}(k)$ is the predicted state estimate at the sampling instant $k$, and $\mathbf{q}(k)$ is the actual state estimate. $\mathbf{G}(k)$ is the Kalman gain. $\mathbf{M}(k)$ is the 


\subsection{States Observer}

covariance of the prediction errors. Matrices $R_{v}$ and $R_{w}$ are the covariance matrices of the observation noise, and disturbance signals respectively.

The covariance of the input disturbance in a real engine cylinder are not estimated in our research. A large numerical value is used in order to ensure that sufficient robustness towards disturbances is achieved. The actual value of the covariance of the input disturbance was determined by observing the control stability of the actuator when exposed to different types of friction forces.

Generally, the estimation of $R_{v}$ is based on the assumption that it is true random noise with a Gaussian form and a standard deviation comparable to the size of the least significant bit of the sensor. Then the matrix has the following form:

$$
R_{v}=\operatorname{cov}(\theta)=\left[E\left(\theta^{2}\right)\right]
$$

For sensor quantization, the error probability for any one sample is uniform within the region -0.5 to 0.5 , and zero elsewhere. The covariance of the noise for one sample can be calculated as:

$$
\begin{aligned}
R_{v} & =\int_{-\infty}^{\infty} \int_{-\infty}^{\infty} x p_{\theta}(x) \times x p_{\theta}(x) d x d x \\
& =\int_{-0.5}^{0.5} \int_{-0.5}^{0.5} x p_{\theta}(x) \times x p_{\theta}(x) d x d x \\
& =\frac{2(0.5)^{3}}{3}=0.0833
\end{aligned}
$$

where, $p_{\theta}(x)$ is the function of probability.

The Kalman Filter is simulated in Simulink together with other system components. Simulation results comparing the performance of different filtering techniques are given in chapter 7 . They show the advantage of the Kalman Filter over direct differentiation and low pass filtering. 


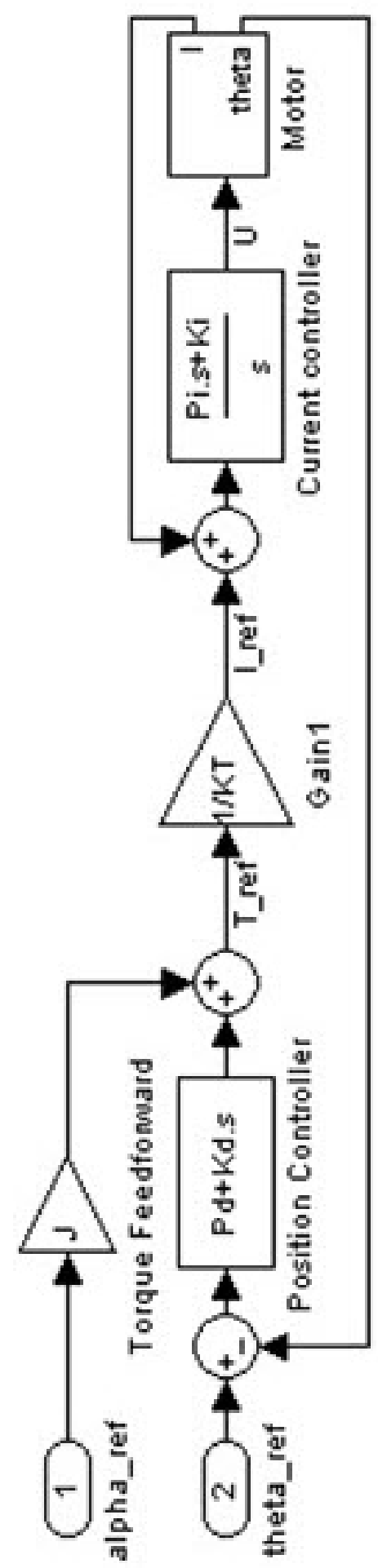

Figure 6.2: Cascaded control structure 


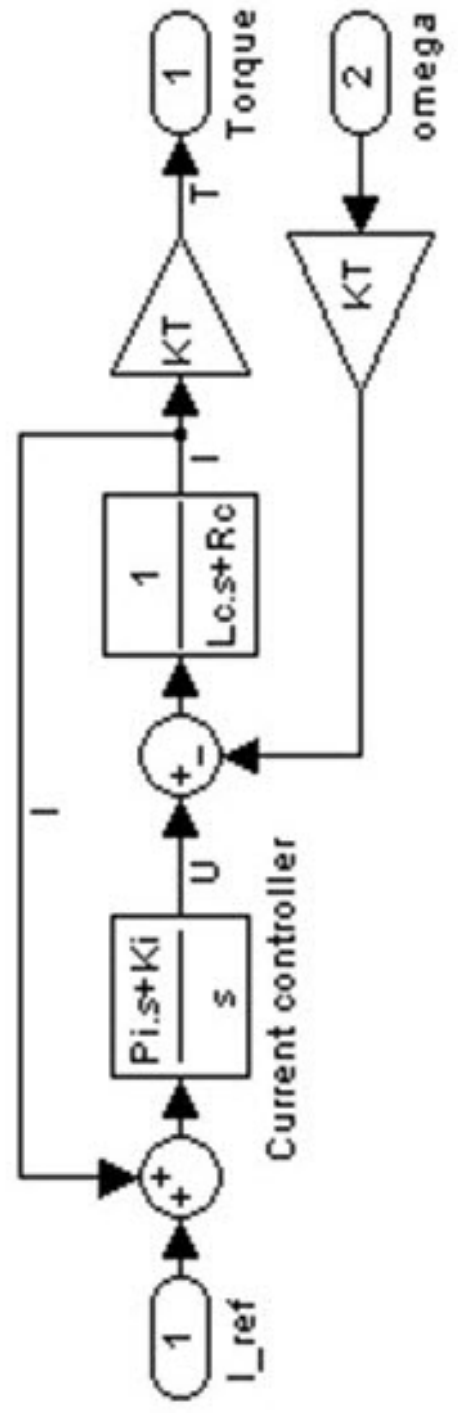

Figure 6.3: Current controller 


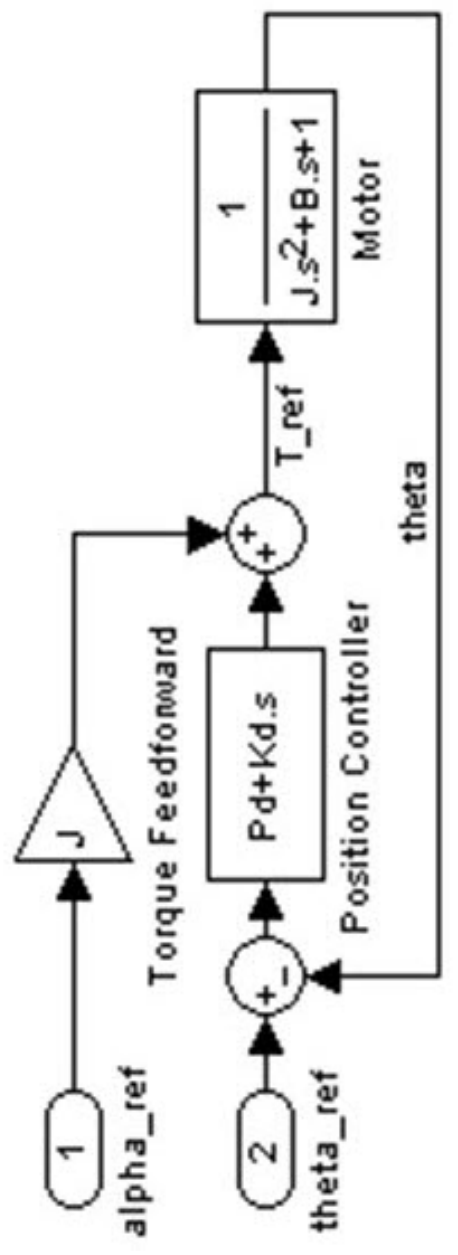

Figure 6.4: Position controller 


\section{Chapter 7}

\section{System Simulation and Experimental Validation}

In the previous chapters, two setups were introduced. The first setup uses a stock QB02302 motor, and the second setup uses an optimized custom motor that provides lower power consumption.

The first part of this chapter describes the components of an experimental test bed used in this study. The second part of this chapter compares the transition performance of the two motors using a Simulink simulation for typical engine operating scenarios. The third part of this chapter looks at the robustness of the actuation system towards parameter variations of the mechanical design and variations of the motor parameters. The fourth part of this section focusses on the ability of the control system to reject external disturbances due to combustion pressures or friction. Finally, the fifth part of this chapter describes the experimental results obtained with the stock QB02302 setup.

\subsection{Experimental Setup}

The experimental test bed drives a single exhaust valve of a Honda cylinder head (see Figure 7.1). A block diagram of the FFVA system is shown in Figure 6.1. It contains a dSpace1103 controller board that creates trajectories and performs position control. For position measurements, a 20000 line encoder from Quantum devices is used. The control board performs its tasks at $40 \mathrm{kHz}$ in order to match the PWM frequency of the subsequent 3 phase inverter from Maccon $\mathrm{GmbH}$. The inverter performs current control and commutation using high speed PWM controlled power MOSFETs that can regulate up to $100 \mathrm{~A}$ per phase. The inverter internally uses LEM modules to feed the current controllers. In addition, three external LEM HTP100- 


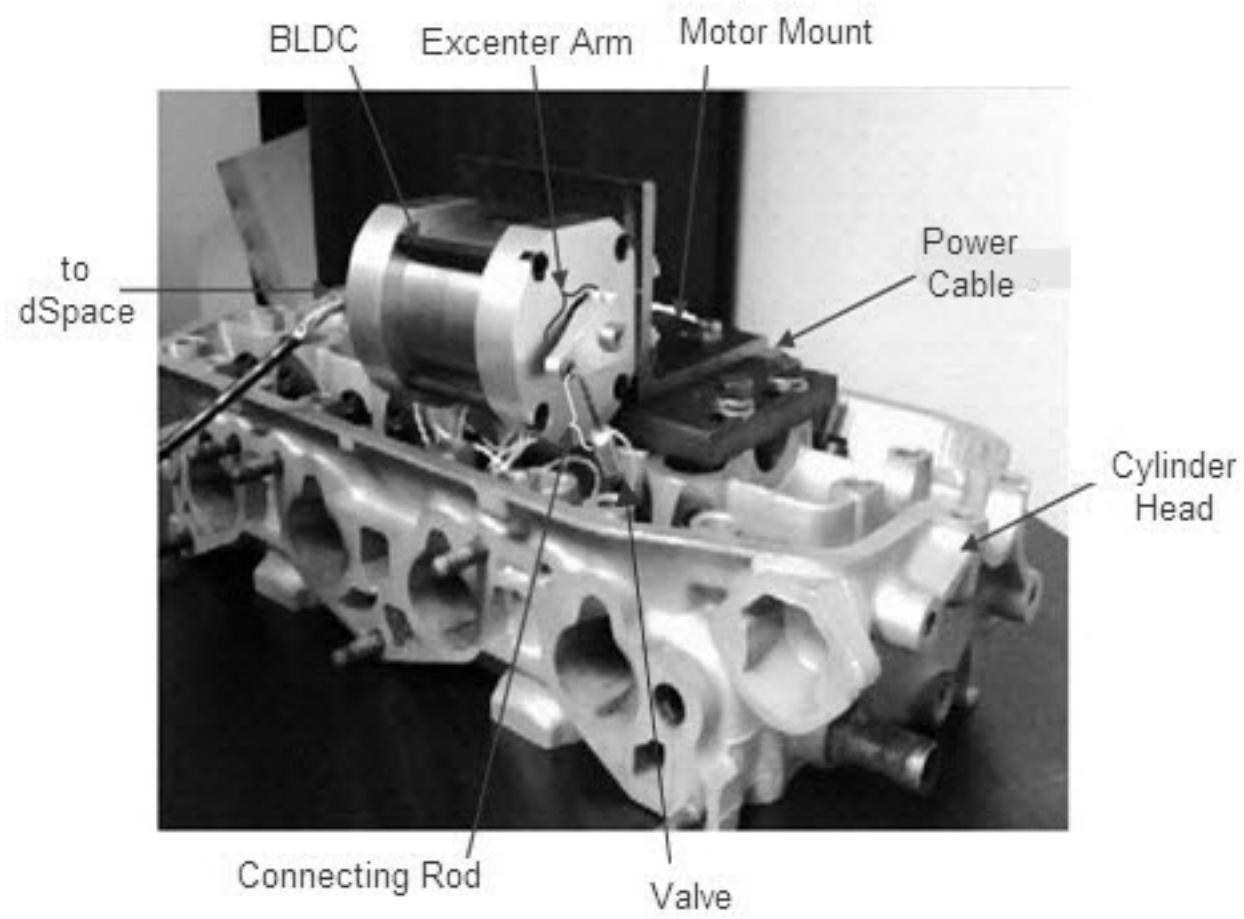

Figure 7.1: System hardware

$\mathrm{P}$ current probes are employed to measure and display currents with the dSpace system. The inverter drives a QB02302 motor that is mounted to a small cylinder head from Honda. The light weight excenter arm is fabricated in aluminum and has a length of $23 \mathrm{~mm}$ in order to match the $37 \mathrm{~g}$ valve to the inertia of the motor. The excenter arm and the valve are joined using a connecting rod made from $2 \mathrm{~mm}$ piano wire. Table 7.1 lists the parameters (QB02302 with customized winding) of the FFVA system.

\subsection{Simulation of Transition Performance}

Before building an experimental setup, the overall system is simulated using Simulink. All mechanical, electrical and magnetic components of the system are included in this simulation. 


\begin{tabular}{|l||l|l|}
\hline \multicolumn{3}{|c|}{ QB02302 with customized winding } \\
\hline Torque Constant $K_{T}$ & 0.056 & Volts \\
Back EMF constant $K_{B}$ & 0.056 & $\mathrm{~V} / \mathrm{rad} / \mathrm{s}$ \\
Peak Current $I_{p}$ & 100 & Ampere \\
Terminal Resistance $R$ & 0.13 & Ohms \\
Terminal Inductance $L$ & 0.15 & $\mathrm{mH}$ \\
Wire Diameter $d_{w}$ & 0.7229 & $\mathrm{~mm}$ \\
Motor Inertia $J_{m}$ & $2.3 \mathrm{E}-5$ & $\mathrm{~kg} \cdot \mathrm{m}^{2}$ \\
Airgap Flux Density $B_{g}$ & $\sim 0.9$ & Tesla \\
Magnet Thickness $l_{m}$ & 3.5 & $\mathrm{~mm}$ \\
Effective Airgap $l_{g e}$ & 4.05 & $\mathrm{~mm}$ \\
Stator Inner Radius $r_{s i}$ & 14.5 & $\mathrm{~mm}$ \\
Stator Outer Radius $r_{s o}$ & 27.5 & $\mathrm{~mm}$ \\
Motor Length $l$ & 56 & $\mathrm{~mm}$ \\
Number of Phases & 3 & \\
Number of Poles & 6 & \\
Number of Slots & 9 & \\
Number of Conductors per Slot & 36 & \\
\hline
\end{tabular}

Table 7.1: Motor Parameters

Two separate simulations are performed. First, the stock motor QB02302 system is simulated and its performance is estimated. Second, the optimized motor is simulated in order to compare its possible performance improvement with the predictions from the design procedure.

The transition performance of the closed loop controlled system can be quantified by using the following three indices [45]:

Transition Time: The time it takes for the valve to move from $5 \%$ of the maximum lift to $95 \%$ of the maximum lift.

Valve Seating Velocity: The velocity of the valve when it contacts the valve seats.

Energy Loss: The copper loss per valve per transition.

The initial simulation of the actuation system with the stock QB02302 motor is based on the parameters listed in Table 7.1.

A number of different operating conditions are simulated to demonstrate the flexibility and performance of the FFVA system. Figure 7.2 shows the 
simulated valve lift curves and Figure 7.3 depicts the corresponding energy consumptions.

The dark blue curve shows a typical $8 \mathrm{~mm}$ valve lift curve for $6000 \mathrm{rpm}$, which corresponds to $t_{2}=2.4 \mathrm{~ms}$. As predicted in Section 3.4, this trajectory requires $1.35 \mathrm{~J} /$ transition which is slightly more than the desired $1.25 \mathrm{~J} /$ transiton. This problem can be alleviated by reducing the lift. A trajectory with half the lift and the same transition time is shown in green and it requires approximately $0.35 J$ which is less than a third of the energy consumed for twice the lift.

In practice, the required lift reduction would be much smaller in order to achieve the desired energy consumption. The red curve shows an $8 \mathrm{~mm}$ lift curve for 3000rpm. Since the additional valve open time was achieved by inserting extra time with the valve completely open, rather than reducing the valve accelerations, the energy consumption is still approximately $1.35 \mathrm{~J}$ in every transition. In practice, one would likely reduce the acceleration in order to find a compromise between engine performance and valve energy loss. The light blue curve represents a $4 \mathrm{~mm}$ valve lift curve at $8000 \mathrm{rpm}$. Compared to the case of $6000 \mathrm{rpm}$ with the same lift, this mode requires

almost three times the energy. This highlights the nonlinear relationship between energy consumption and transition time.

Even though the setup with the stock QB02302 motor performs quite well in the simulations, the energy consumption at high rpm is slightly larger than desired. The optimized custom motor was designed to address this problem in Chapter 4. Figure 7.4 shows the displacement curves and energy consumption for simulating the optimized motor at 6000rpm engine speed. The energy consumption for this setup has been reduced from $1.35 \mathrm{~J} /$ transtion to $0.66 \mathrm{~J} /$ transition. This corresponds well with the predictions shown in Table 5.1.

\subsection{Simulation of the Robustness Towards Parameter Variations}

The system parameters such as valve mass $m$, excenter arm length $r_{a}$, motor torque constant $K_{T}$ and winding resistance $R$ can shift from the nominal values due to manufacture accuracy, abrasion, temperature or other factors. In the simulation, the values of the listed four parameters are all increased 


\subsection{Simulation of the Robustness Towards Parameter Variations}

by $30 \%$ from the nominal value. Each parameter is varied individually in one simulation.

The results of these simulations are shown in Figure 7.5 and they demonstrate that the control system is able to compensate for a wide range of parameter variations, since all the motion curves including position, velocity and acceleration are still accurately following the reference trajectories. However, energy losses are different. The sensitivity of the energy consumption towards parameter variations can be estimated by taking partial derivatives of Equation 3.10 with respect to the parameters being varied. For the parameters studied here this leads to:

$$
\begin{gathered}
\frac{\delta E}{E} \approx \frac{\frac{\partial E}{\partial m} \delta m}{E}=\frac{\delta m}{m} \\
\frac{\delta E}{E} \approx \frac{\frac{\partial E}{\partial R} \delta R}{E}=\frac{\delta R}{R} \\
\frac{\delta E}{E} \approx \frac{\frac{\partial E}{\partial K_{t}} \delta m}{E}=-2 \frac{\delta K_{t}}{K_{t}} \\
\frac{\delta E}{E} \approx \frac{\frac{\partial E}{\partial r_{a}} \delta r_{a}}{E}=0
\end{gathered}
$$

Note that since the optimum excenter arm length was chosen to provide minimum energy consumption, the derivative of the energy with respect to arm length and hence the sensitivity in Equation 7.4 need to be zero. It is

\begin{tabular}{|l||l|l|l|}
\hline parameter & $\begin{array}{l}\text { stationary } \\
\text { point }\end{array}$ & $\begin{array}{l}\text { estimated sensitivity } \\
\frac{\delta E}{E}\end{array}$ & $\begin{array}{l}\text { simulated } \\
\text { sensitivity }\end{array}$ \\
\hline Valve mass $m$ & $0.037 \mathrm{~g}$ & $30 \%$ & $38 \%$ \\
Motor resistance $R$ & $0.13 \mathrm{Ohm}$ & $30 \%$ & $31 \%$ \\
Torque constant $K_{t}$ & $0.056 \mathrm{Nm}$ & $-60 \%$ & $-42 \%$ \\
Excenter arm length $r_{a}$ & $0.023 \mathrm{~m}$ & 0 & $10 \%$ \\
\hline
\end{tabular}

Table 7.2: Sensitivity

expected that actual sensitivity values will differ from the nominal values, because large changes are made to the parameters and Equations 7.1 to 7.4 provide only a linearized approximation. Table 7.2 shows a comparison of the estimated and the simulated sensitivity values. It demonstrates that the torque constant, the resistance, and the valve mass have a large influence on 
the energy consumption, whereas the excenter arm length is not very sensitive. This lets us conclude, that manufacturing tolerances of the linkage system do not need to be very high in order to guarantee low energy consumption. On the other hand all the other parameters need to be designed carefully.

\subsection{Simulation of Disturbance Rejection}

The FFVA system is designed for intake valves where only moderate combustion pressure is expected on the valves. The shape of the pressure curve during the opening or the closing transition is not well defined. Other disturbances could include some degree of viscous damping or coulomb damping in the motor or in the valve guides. In this investigation we attempt to combine all of these causes using disturbance torques on the motor that are modeled with a viscous friction coefficient of $0.015 \mathrm{Nms} / \mathrm{rad}$ and a coulomb coefficient of approximately $1.15 \mathrm{Nm}$. Each of the two parameters corresponds to an equivalent disturbance force of $50 \mathrm{~N}$ on the valve.

It should be pointed out, that the original controller was not designed to compensate for disturbances in the resting positions at either end of the valve motion. In order to achieve low steady state errors under these circumstances, the original PD position controller needs to be extended to a PID controller. The I term is used to reduce steady state error and the $\mathrm{PD}$ terms are defined in the same fashion as in the original design.

Figure 7.6 shows simulation results that indicate that the system is able to cope with significant disturbance forces. The additional energy required by the actuation system is very close to the energy dissipated in friction. We conclude, that the efficiency of the actuation system in the presence of disturbances is still close to optimal.

\subsection{Simulation of Low Resolution Sensor with a Kalman Filter}

The experimental system used in this thesis uses a very high resolution optical encoder. In a real engine, this is not a suitable sensor, because it is too expensive and cannot withstand the harsh environmental conditions. A hall sensor is a more likely candidate, and it would usually have a resolu- 
tion somewhere between 7 and 10 bits. At the high sampling rates used for this system, the low resolution would lead to considerable quantization error, that would consequently introduce large noise in the position signal. For eliminating measurement noise we will now compare a low pass filter and a Kalman filter. The low pass filter is a simple backward difference implementation of a continuous first order lag with a break frequency that is ten times higher than the position loop bandwidth. The Kalman filter implementation and the selection of the covariances for the disturbances and the measurement follow the guidelines shown in Chapter 6. Figure 7.7 shows the systems response using an 7 bit sensor with 1 bit measurement noise and different feedback strategies. When the sensor signal and a direct backwards differentiator are used, the system is unstable. When the sensor and the backwards differentiator are combined with low pass filters with bandwidths at 10 times the bandwidth of the position controller, the oscillations are reduced but not eliminated. Only the Kalman filter, is able to provide a stable response for the low resolution sensor.

The reason for the difference in control performance can be found by comparing position and velocity errors for the different feedback signals. Figure 7.8 then shows this comparison. As expected, the position error of the Kalman filter is slightly smaller than the actual sensor signal. However, the low pass filter demonstrates considerable phase lag which manifests itself in a position error proportional to velocity. The velocity error of the direct backward difference differentiator is the same size as the full scale velocity measurement. This error is reduced considerably by both the low pass filter and the Kalman filter. However, the latter shows less than half the noise levels of the low pass filter.

In summary, for a low resolution sensor system, the Kalman filter is the only approach investigated here that guarantees sufficient robustness.

\subsection{Experimental Results}

In this section, the experimental system performance of the stock QB02302 motor is compared with the simulations and with literature data from EMCV and EMVD systems. In these experiments, the valve opens $8 \mathrm{~mm}$ in approximately $3.4 \mathrm{~ms}$, which corresponds to a typical valve motion at a $6000 \mathrm{rpm}$ engine speed.

Figure 7.9 shows position, velocity and acceleration of the valve. Track- 
7.6. Experimental Results

\begin{tabular}{|l||l|l|l|l|}
\hline Lift $=8 \mathrm{~mm}$ & $\begin{array}{l}\text { EMCV1 } \\
{[46]}\end{array}$ & $\begin{array}{l}\text { EMCV2 } \\
{[47]}\end{array}$ & $\begin{array}{l}\text { EMVD } \\
{[14]}\end{array}$ & FFVA \\
\hline $\begin{array}{l}\text { Transition } \\
\text { Time }[\mathrm{ms}]\end{array}$ & 3.5 & 3.5 & 3.4 & 3.4 \\
\hline $\begin{array}{l}\text { Seating } \\
\text { Velocity }[\mathrm{m} / \mathrm{s}]\end{array}$ & 0.3 & 0.1 & $0.15 \sim 0.27$ & $0.1 \sim 0.25$ \\
\hline $\begin{array}{l}\text { Energy Loss } \\
{[\mathrm{J} / \text { transition }]}\end{array}$ & $3.0 \sim 5.2$ & - & 1.4 & $\begin{array}{l}1.35(\mathrm{QB} 02302) \\
0.66(\text { Optimized })\end{array}$ \\
\hline $\begin{array}{l}\text { Voltage } \\
\text { Source }[\mathrm{V}]\end{array}$ & 100 & 42 & 42 & 42 \\
\hline $\begin{array}{l}\text { Controllable } \\
\text { Variables }\end{array}$ & $\begin{array}{l}\text { Duration } \\
\text { \& Phase }\end{array}$ & $\begin{array}{l}\text { Duration } \\
\& \text { Phase }\end{array}$ & $\begin{array}{l}\text { Duration } \\
\& \text { Phase }\end{array}$ & $\begin{array}{l}\text { Fully Variable } \\
\text { \& Programmable }\end{array}$ \\
\hline
\end{tabular}

Table 7.3: Performance comparison

ing performance is quite good. However, due to the flexibility in the linkage between valve and motor, mechanical vibrations are induced. This cause was determined by comparing the standard operation to a setup with an equivalent inertia mounted rigidly to the motor. With the rigid inertia, no noticeable vibration was observed. This leads to the conclusion that the rather rudimentary linkage implementation shown here needs to be redesigned to provide less flexibility in future setups. The velocity plot shows that the mechanical vibrations due to the flexible link are primarily excited during the high jerk periods at the beginning and at the end of the motion. Consequently, the seating velocity at the end of the valve travel is rather high. It should be noted that the transition time chosen here would be used during high rpm of the combustion engine. However, the low valve seating velocity of $0.05 \mathrm{~m} / \mathrm{s}$ is only required during engine idle, when the transition times are much longer and the valve lift can be reduced significantly. Under such circumstances, the FFVA system will provide valve seating velocities well below the desired value.

Phase current, total current, and energy loss are plotted in Figure 7.10. The motor draws approximately $100 \mathrm{~A}$ during acceleration and $60 \mathrm{~A}$ during deceleration. The unsymmetrical behavior and the large current oscillations during accelerations are again attributed to the flexible linkage system, which has unsymmetrical damping elements for opening and closing. However, the energy consumption of $1.35 \mathrm{~J} /$ transition in the experiment corresponds well to the analytical predictions in Chapter 4. Table 7.3 shows 


\subsection{Experimental Results}

a comparison of the performance characteristics of the FFVA system with the EMVD and the EMCV system. For a similar transition time and lift, the FFVA system performs as good or better than the other two systems. However, the FFVA system provides not only variable valve timing but also variable lift. This additional flexibility leads to significant operating advantages for the combustion engine. 


\subsection{Experimental Results}

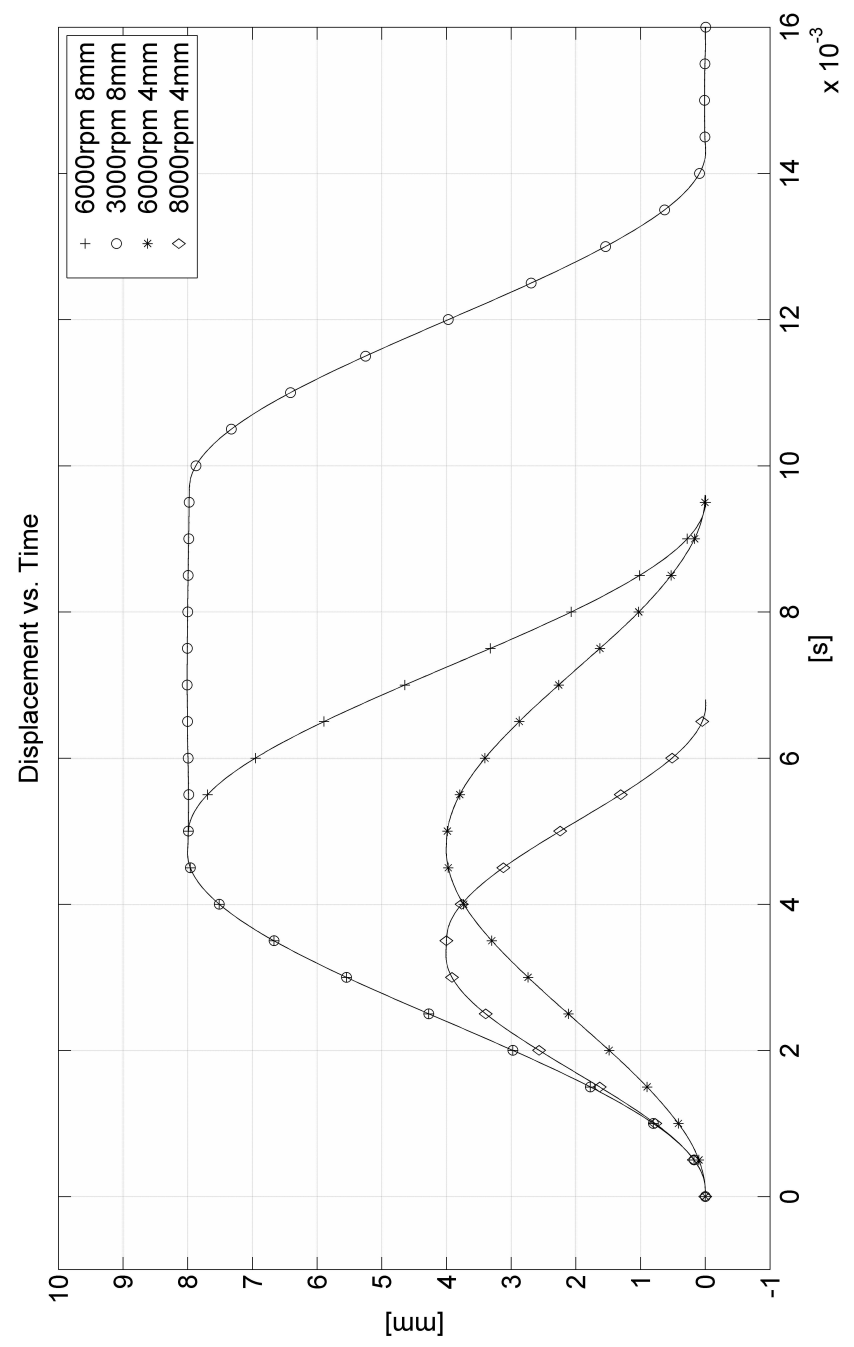

Figure 7.2: Simulation of displacement trajectories 


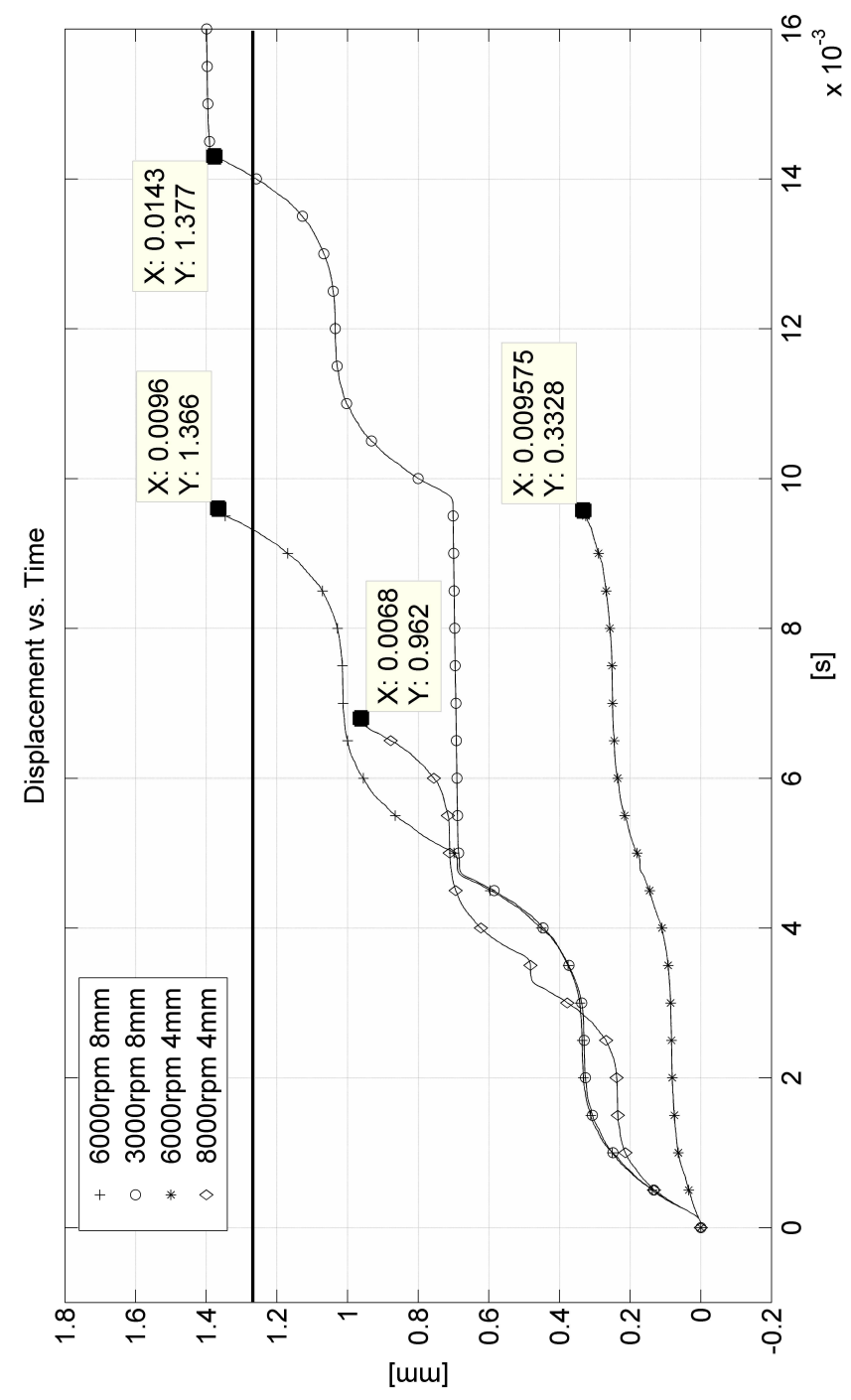

Figure 7.3: Simulation of energy losses of different trajectories 

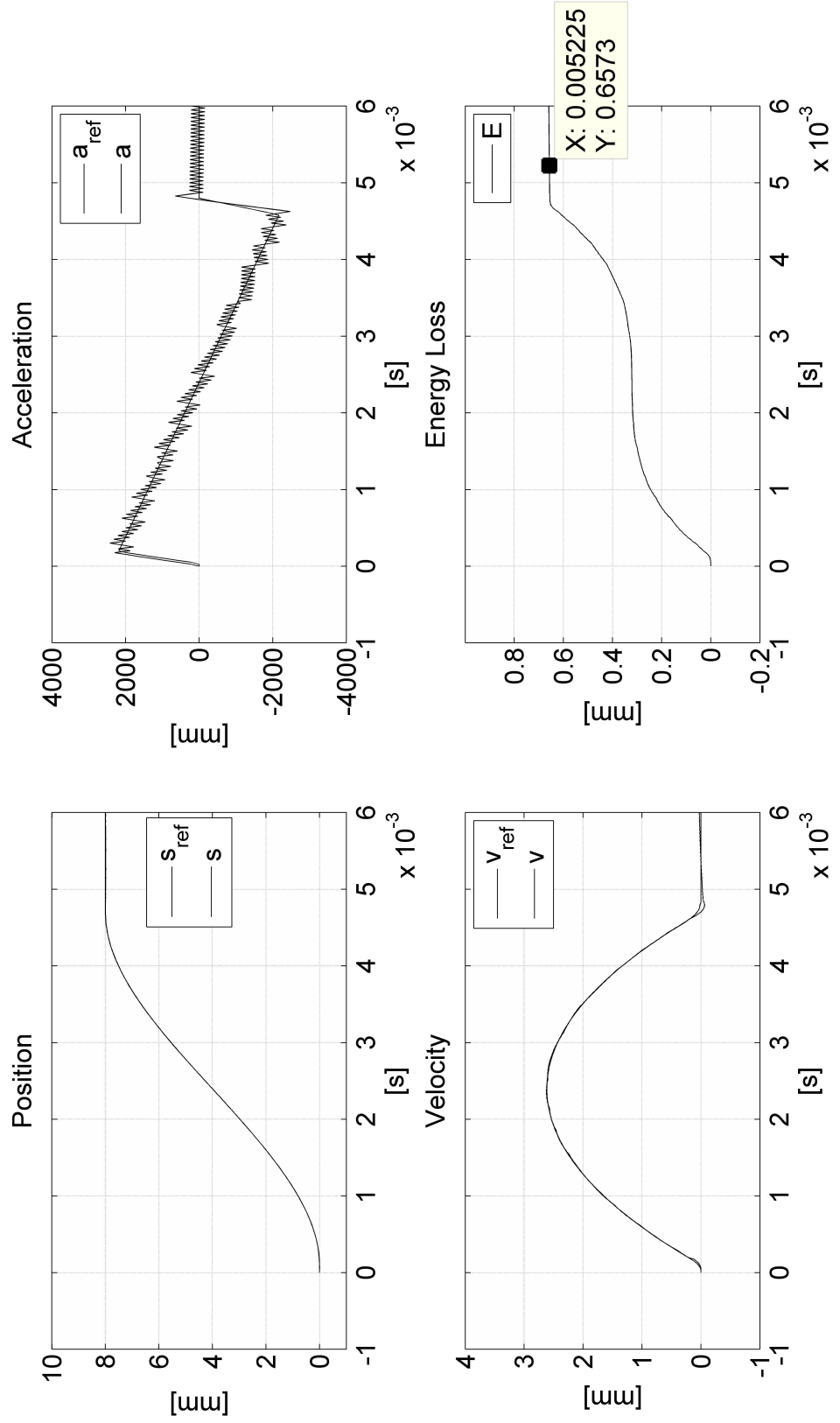

Figure 7.4: Transition simulation of the optimized motor 

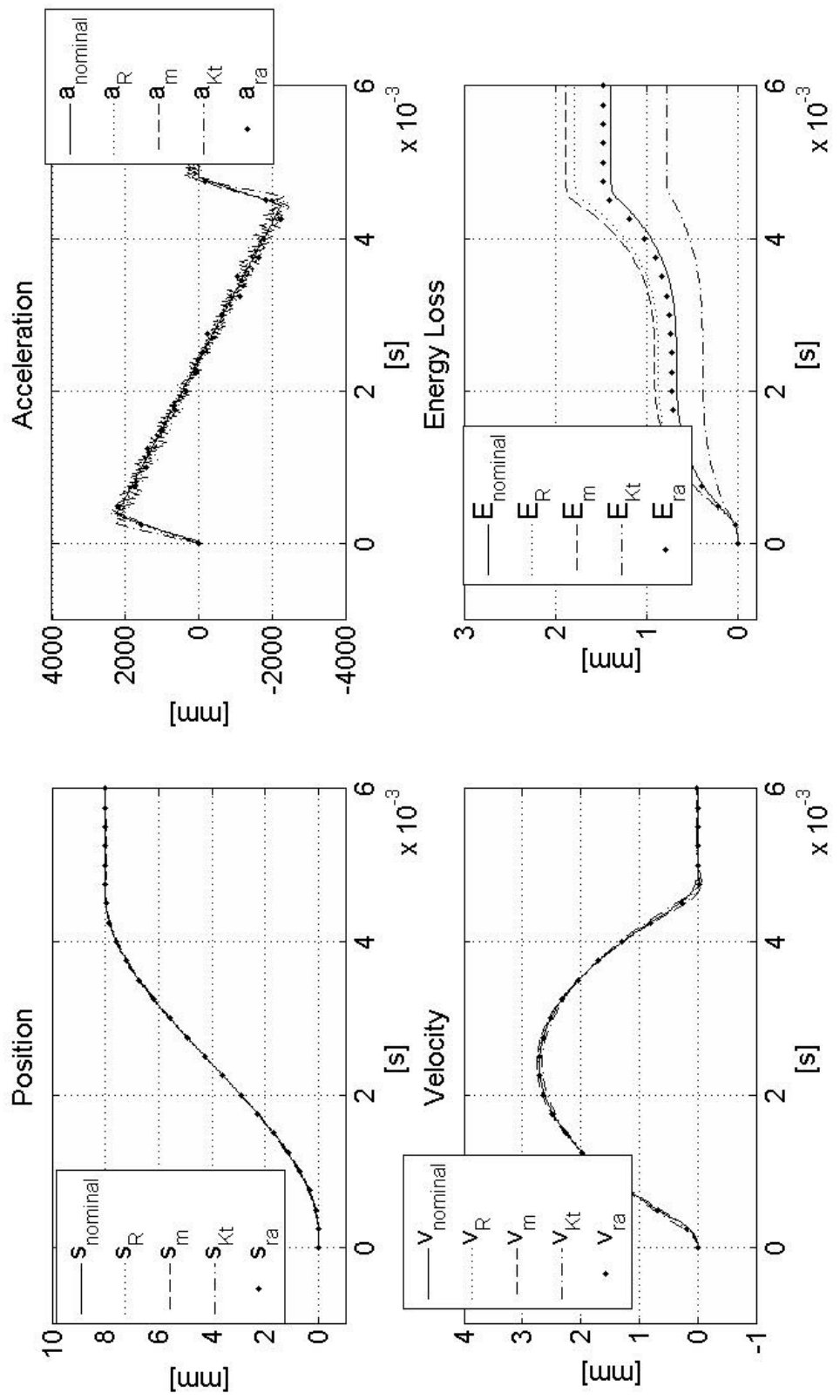

Figure 7.5: Simulation of parameter variations 

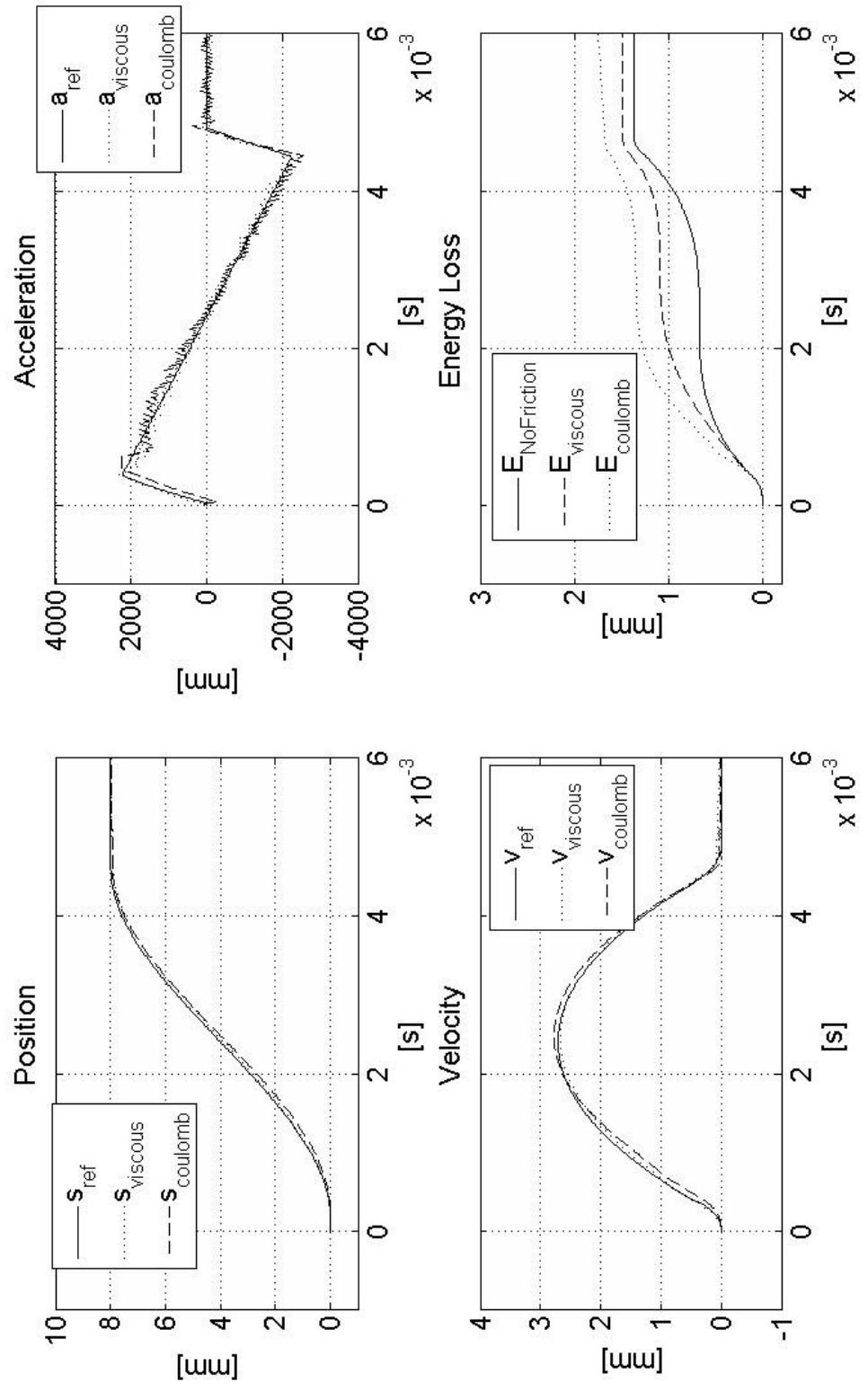

Figure 7.6: Simulations of disturbance rejection 


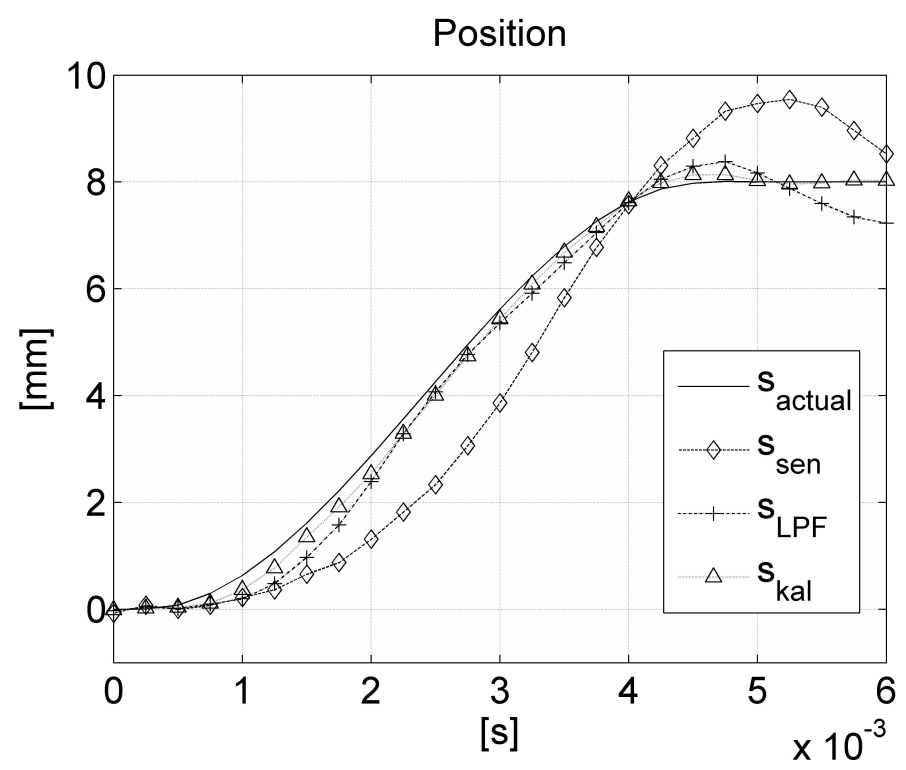

Figure 7.7: Simulation of transition response with a 7 bit sensor and various filtering strategies 


\subsection{Experimental Results}
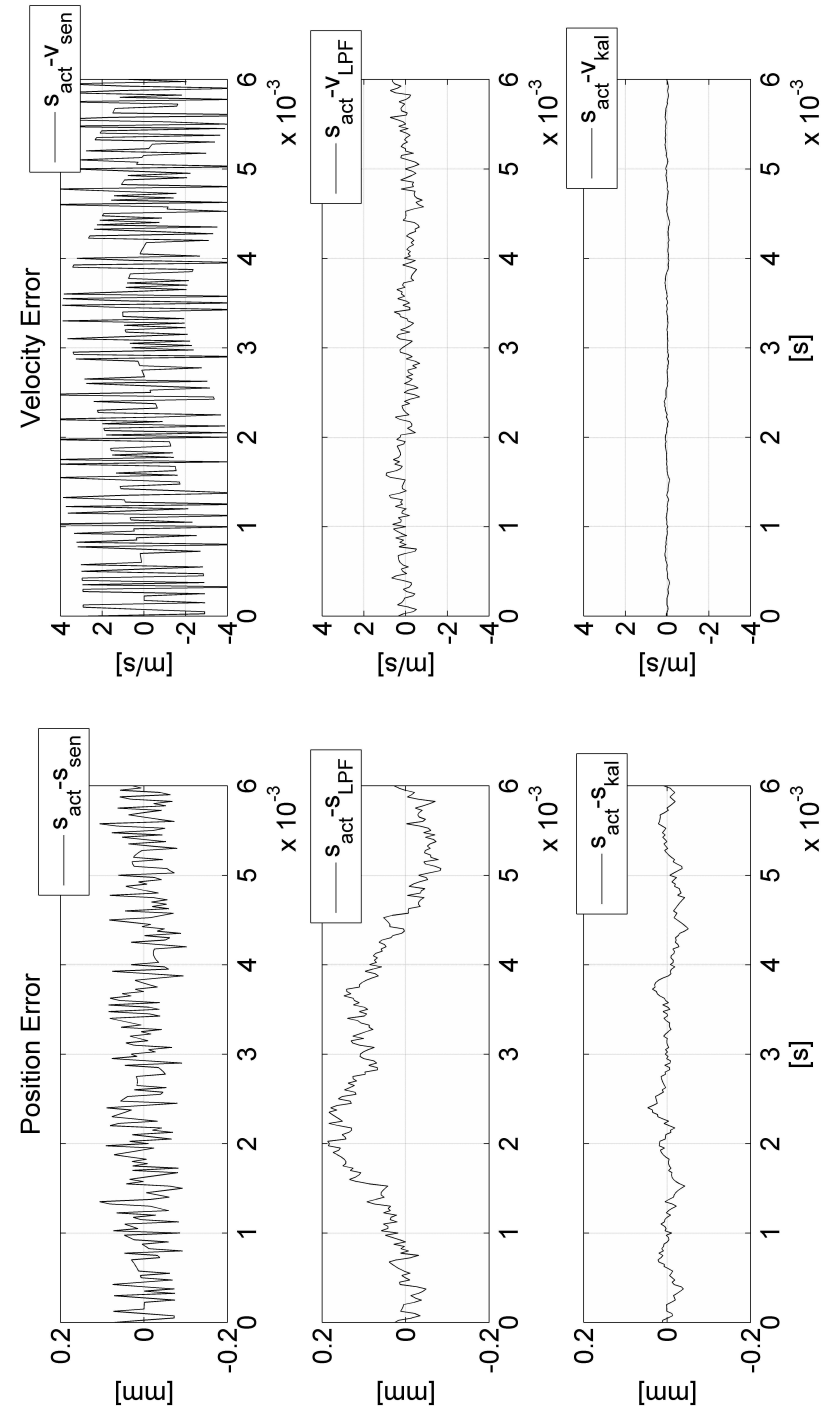

Figure 7.8: Simulations of measurement error using different filtering strategies 


\subsection{Experimental Results}
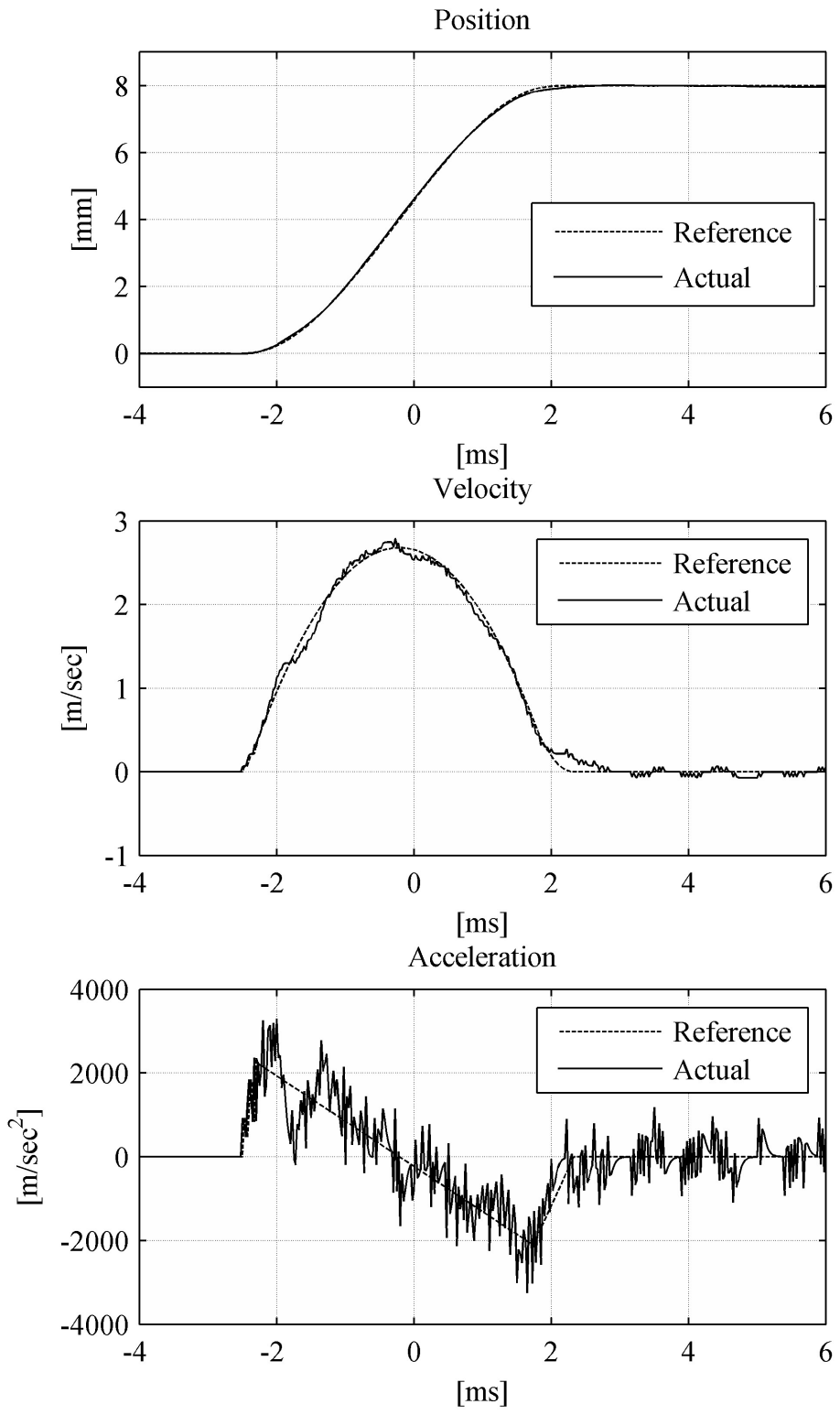

Figure 7.9: Experimental trajectory results using QB02302 


\subsection{Experimental Results}

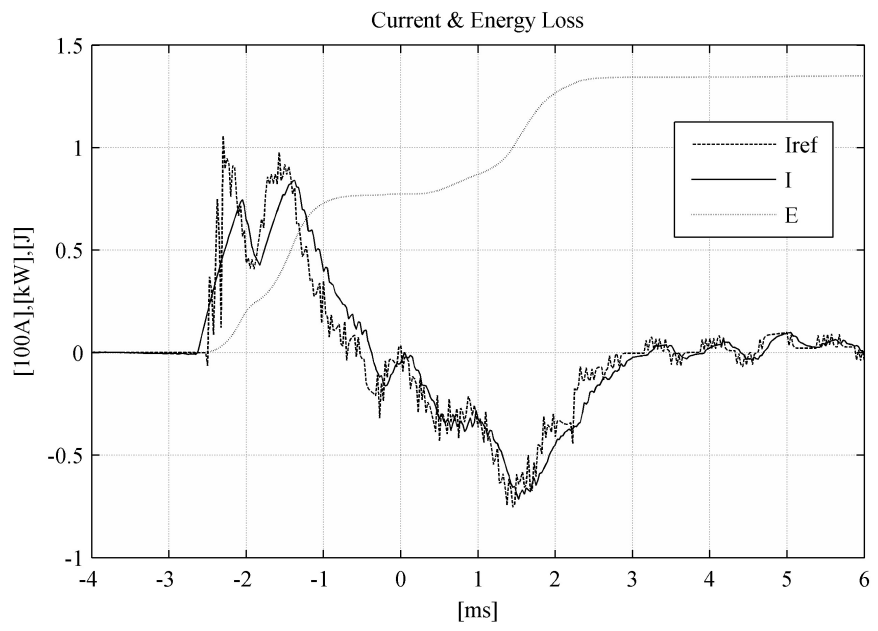

Figure 7.10: Experimental energy loss using QB02302 


\section{Chapter 8}

\section{Conclusion}

\subsection{Summary}

In this thesis, a Fully Flexible Valve Actuation system for the intake valves of automotive internal combustion engines is demonstrated.

After reviewing the need for electronic valve actuation and current implementations of such systems, a direct drive system is proposed that provides six main advantages:

- Fully flexible valve control

- Simple mechanical layout

- An effective control system

- Good energy efficiency: 1.35J/transition using QB02302 (simulation results show that the energy loss is only $0.66 \mathrm{~J} /$ transition using an optimized motor)

- Fast transition: sufficient for the operation at 6000rpm engine speed

- Relatively low seating velocity, which is regulated to about $0.2 \mathrm{~m} / \mathrm{s}$ at $6000 \mathrm{rpm}$ and has the potential to be improved. Also, at engine idle the system can provide the desired seating velocity of $0.05 \mathrm{~m} / \mathrm{s}$.

For a successful implementation, all aspects of the actuation system need to be optimized. This includes the mechanical portion, the electromag- 
netic portion, and the control algorithms of the actuation system. A design methodology for an actuation system based on stock motors is proposed first. It provides guidelines how to select a good motor, how to design the mechanical layout, and how to design an energy efficient control strategy. In the course of this work, analytical expressions were developed to predict the transition performance and the energy efficiency of the actuation system. In addition, a lumped parameter model was developed to support the analytical performance predictions. The lumped parameter model was also used to study the influence of varying system parameters, the ability to reject disturbances, and effects of sensors with low resolution. Finally a test bed was constructed that was used to validate the transition performance. These investigations show that the FFVA system based on stock motors perform well compared to other systems found in the literature.

To further improve the performance of the FFVA system, an analytical design methodology for modifying the stock motors is then proposed. An FEM model is used to validate the motor optimization methodology and the lumped parameter model is applied again in order to predict the system performance. This study shows that the modified motor should provide superior energy efficiency without compromising transition speed.

\subsection{Suggestions for Future Works}

Even though the current experimental setup performs well compared to other systems found in literature, there are still a number of areas of improvement:

As shown in Figure 7.9, the seating velocity is still relatively high. This is likely due to the elasticity of the connecting rod that causes a bounce when the valve comes to the end of a transition. A stiffer joint needs to be designed, or the control system needs to be refined in order to reduce the seating velocity.

A second area of work is the experimental implementation of the optimized custom motor. The theocratical derivation and simulation of the optimized motor have been presented in this thesis, and this motor promises to improve the performance of the actuation system significantly. However, the scope of the thesis did not allow for an experimental implementation.

Finally, the current system is powered by a voltage source of $42 \mathrm{~V}$. For 


\subsection{Suggestions for Future Works}

better compatibility with current vehicles, the supply voltage needs to be decreased to $12 \mathrm{~V}$. Further optimization has to be performed on the current system structure to meet this requirement. 


\section{Bibliography}

[1] M. Forissier, "Reducing greenhouse gases," Forum Annuel de IInvestissement Responsable en Europe, sponsored by French SIF, Tech. Rep., June 2007.

[2] N. Milovanovic, R. Chen, and J. Turner, "Influence of variable valve timings on the gas exchange process in a controlled auto-ignition engine," in Proceedings of the Institution of Mechanical Engineers Part D-Journal of Automobile Engineering, vol. 218, 2004, pp. 567-583.

[3] R. R. Henry and B. Lesquesne, "A novel, fully-flexible, electromechanical engine valve actuation system," SAE Tech. Paper Series, 1997.

[4] S. Butzmann, J. Melbert, and A. Koch, "Sensorless control of electromagnetic actuators for variable valve train," SAE Tech. Paper Series, 2000 .

[5] E. Sher and T. Bar-Kohany, "Optimization of variable valve timing for maximizing performance of an unthrottled si engine - a theoretical study," Energy, 2002.

[6] R. R. Chladny, C. R. Koch, and A. F. Lynch, "Modeling automotive gas-exchange solenoid valve actuators," IEEE Transactions On Magnetics, vol. 41, no. 3, March 2005.

[7] J. G. Calvert, J. B. Heywood, R. F. Sawyer, and J. H. Seinfeld, "Achieving acceptable air quality: Some reflections on controlling vehicle emissions," Science, vol. 261, no. 5117, pp. 37-45, July 1993.

[8] M. Pischinger, W. Salber, F. V. D. Staay, H. Baumgarten, and H. Kemper, "Low fuel consumption and low emissions electromechanical valve train in vehicle operation," International Journal of Automotive Technology, vol. 1, no. 1, pp. 17-25, September 2000. 
[9] M. Theobald and B. L. and.R. Henry, "Control of engine load via electromagnetic valve actuators," SAE Tech., vol. 103, pp. 1323-1334, 1994.

[10] V. A. W. Hillier and F. W. Pittuck, Fundamentals of motor vehicle technology. Hutchinson, 1966.

[11] W. Hoffmann and A. G. Stefanopoulou, "Valve position tracking for soft landing of electromechanical camless valvetrain," Advances in Automotive Control 2001, pp. 295-300, 2001.

[12] W. S. Chang, T. A. Parlikar, M. D. Seeman, D. J. Perreault, J. G. Kassakian, and T. A. Keim, "A new electromagnetic valve actuator," Ieee Power Electronics in Transporation, pp. 109-118, 2000.

[13] K. Peterson, A. Stefanopoulou, T. Megli, and M. Haghgooie, "Output observer based feedback for soft landing of electromechanical camless valvetrain actuator," in Proceedings of the 2002 American Control Conference, Vols 1-6, 2002, pp. 1413-1418.

[14] T. A. Parlikar, W. S. Chang, Y. H. Qiu, M. D. Seeman, D. J. Perreault, J. G. Kassakian, and T. A. Keim, "Design and experimental implementation of an electromagnetic engine valve drive," IEEE/ASME Transactions On Mechatronics, vol. 10, no. 5, October 2005.

[15] C. F. Taylor, The internal-combustion engine in theory and practice. M.I.T. Press, 1985.

[16] A. Nix, "The future of 42v vehicle systems," Vehicle Systems Integration in the Wired World, pp. 167-176, 2001.

[17] H. T. and H. M., "Development of the variable valve timing and lift (vtec) engine for the honda nsx," SAE transactions, vol. 100, no. 3, pp. $1-7,1991$.

[18] Moriya, Y. Saitoh, T. Kawatake, and M. K. Yoshioka, "Vvt-i system," TOYOTA TECHNICAL REVIEW, vol. 47, no. 1, pp. 48-53, 1997.

[19] J. S. Brader, "Development of a piezoelectric controlled hydraulic actuator for a camless engine," Master's thesis, Boston University, 1995.

[20] Z. Sun and D. Cleary, "Dynamics and control of an electro-hydraulic fully flexible valve actuation system," in Proceedings of the American Control Conference, Denver, Colorado, June 2003, pp. 3119-3124. 
[21] C. Tai, T.-C. Tsao, and M. B. Levin, "Adaptive nonlinear feedforward control of an electrohydraulic camless valvetrain," in Proceedings of the American Control Conference, June 2000.

[22] P. K. Wong, L. M. Tam, and K. Li, "Modeling and simulation of a dualmode electrohydraulic fully variable valve train for four-stroke engines," International Journal of Automotive Technology, vol. 9, pp. 509-521, 2008.

[23] J. M. Donaldson, "Dynamic simulation of an electrohydraulic open center gas-change valve actutor system for camless internal combustion engines," Master's thesis, Milwaukee School of Engineering, 2003.

[24] S. K. Chung, C. R. Koch, and A. F. Lynch, "Flatness-based feedback control of an automotive solenoid valve," IEEE Transactions On Control Systems Technology, vol. 15, no. 2, March 2007.

[25] D. O. Wright and J. A. Nitkiewicz, "Engine valve actuation control system," United States Patent, no. 5632, 1999.

[26] R. Seethaler, J. Meyer, A. Knaut, and K.-H. Gaubatz, "Pivoting actuator for controlling the stroke of a gas exchange valve in the cylinder head of an internal combustion engine," United States Patent, no. 11/128185, 2006.

[27] M. GmbH, "Quantum series brushless servo motors," http://www. maccon.de/FTPROOT/Quantum.pdf, 2009.

[28] Brushless Motors and Drive Systems, Kollmogen Inland Motor.

[29] R. W. J. Armstrong, "Load to motor inertia mismatch:unveiling the truth," in DRIVES AND CONTROLS CONFERENCE, Telford England, 1998.

[30] A. Sasane, "Calculus of variations and optimal control," September 2004.

[31] A. M. Trzynadlowski, "Energy optimization of a certain class of incremental motion dc drives," IEEE Transactions On Industrial Electronics, vol. 35, no. 1, February 1988.

[32] Y. A. Kaan Erkorkmaz, "High speed cnc system design. part iii: high speed tracking and contouring control of feed drives," International Journal of Machine Tools and Manufacture, vol. 41, no. 2001, p. 1637C1658, October 2000. 
[33] Pittman, "64mm series brushless dc motor," http://www.ametektip. com $/$ index.php? option $=$ com_rokdownloadsEview $=$ fileE⿱丶万 id $=1454$, 2009.

[34] MCG, "Motor i2383092nc-xxxx," http://www.ametektip.com/index. php? option=com_contentEview $=$ articleEGid=192, 2009.

[35] R. H. Engelmann and W. H. Middendorf., Handbook of Electric Motors. Dekker, 1995.

[36] G. Slemon, "On the design of high-performance surface-mounted pm motors," IEEE Transactions on Industry Applications, vol. 30, no. 1, pp. 134-140, Jan/Feb 1994.

[37] T. Sebastion and G. R. Slemon, "Transient modeling and performance of variable speed permanent magnet motors," IEEE Transactions on Industry Applications, vol. 25, no. 1, Jan/Feb 1989.

[38] Y. Chin, W. Arshad, T. Backstrom, and C. Sadarangani, "Design of a compact bldc motor for transient applications," in European Coference on Power Electronics, Austria, 2001.

[39] N. Bianchi and S. Bolognani, "Fractional-slot pm motors for electric power steering systems," International Journal of Vehicle Autonomous Systems, vol. 2, no. 3-4, pp. 189-200, 2004.

[40] P. Technology, "Wire gauge and current limits," http://www. powerstream.com/Wire_Size.htm, 2009.

[41] Y. Zhang, K. T. Chau, J. Z. Jiang, D. Zhang, and C. Liu, "A finite elementcanalytical method for electromagnetic field analysis of electric machines with free rotation," IEEE Transactions On Magnetics, vol. 42, no. 10, 2006.

[42] J. P. A. Bastos and N. Sadowski, Electromagnetic Modeling By Finite Element Methods. Marcel Dekker, Inc., 2003.

[43] M. Chari, G. Bedrosian, and J. D'Angelo, "Finite element applications in electrical engineering," IEEE TRANSACTIONS ON MAGNETICS, vol. 29, no. 2, 1993.

[44] G. R. Yu, M. H. Tseng, and Y. K. Lin, "Optimal positioning control of a dc servo motor using sliding mode," in Proceedings of the 2004 IEEE International Conference on Control Applications, 2004. 
[45] R. Pierik and J. Burkhard, "Design and development of a mechanical variable valve actuation system," in SAE technical Paper Series, 2000.

[46] Y. Wang, T. Megli, M. Haghgooie, K. Peterson, and A. Stefanopoulou, "Modeling and control of electromechanical valve actuator," Society of Automotive Engineers, vol. 01, no. 1106, 2002.

[47] R.R.Chladny and C.R.Koch, "Flatness-based tracking of an electromechanical variable valve timing actuator with disturbance observer feedforward compensation," IEEE transactions on Control System Technology, vol. 16, no. 652-633, 2008. 